\title{
Single superpartner production at Tevatron Run II
}

\author{
F. Déliot ${ }^{1}$, G. Moreau ${ }^{2}$, C. Royon ${ }^{1,3,4}$ \\ 1: Service de Physique des Particules, DAPNIA \\ CEA/Saclay 91191 Gif-sur-Yvette Cedex, France \\ 2: Service de Physique Théorique \\ CEA/Saclay 91191 Gif-sur-Yvette Cedex, France \\ 3: Brookhaven National Laboratory, Upton, New York, 11973; \\ 4: University of Texas, Arlington, Texas, 76019
}

November 28, 2018

\begin{abstract}
We study the single productions of supersymmetric particles at Tevatron Run II which occur in the $2 \rightarrow 2-$ body processes involving R-parity violating couplings of type $\lambda_{i j k}^{\prime} L_{i} Q_{j} D_{k}^{c}$. We focus on the single gaugino productions which receive contributions from the resonant slepton productions. We first calculate the amplitudes of the single gaugino productions. Then we perform analyses of the single gaugino productions based on the three charged leptons and like sign dilepton signatures. These analyses allow to probe supersymmetric particles masses beyond the present experimental limits, and many of the $\lambda_{i j k}^{\prime}$ coupling constants down to values smaller than the low-energy bounds. Finally, we show that the studies of the single gaugino productions offer the opportunity to reconstruct the $\tilde{\chi}_{1}^{0}$, $\tilde{\chi}_{1}^{ \pm}, \tilde{\nu}_{L}$ and $\tilde{l}_{L}^{ \pm}$masses with a good accuracy in a model independent way.
\end{abstract}

\section{Introduction}

In the Minimal Supersymmetric Standard Model (MSSM), the supersymmetric (SUSY) particles must be produced in pairs. The phase space is largely suppressed in pair production of SUSY particles due to the important masses of the superpartners. The R-parity violating $\left(R_{p}\right)$ extension of the MSSM contains the following additional terms in the superpotential, which are trilinear in the quarks and leptons superfields,

$$
W_{\not_{p}}=\sum_{i, j, k}\left(\frac{1}{2} \lambda_{i j k} L_{i} L_{j} E_{k}^{c}+\lambda_{i j k}^{\prime} L_{i} Q_{j} D_{k}^{c}+\frac{1}{2} \lambda_{i j k}^{\prime \prime} U_{i}^{c} D_{j}^{c} D_{k}^{c}\right),
$$

where $i, j, k$ are flavour indices. These $R_{p}$ couplings offer the opportunity to produce the scalar supersymmetric particles as resonances $[1,2]$. Although the $R_{p}$ coupling constants are severely constrained by the low-energy experimental bounds $[3,4,5]$, the resonant superpartner production reaches high cross sections both at leptonic [6] and hadronic [7] colliders.

The resonant production of SUSY particle has another interest: since its cross section is proportional to a power 2 of the relevant $R_{p}$ coupling, this reaction would allow an easier determination of the $R_{p}$ couplings than the pair production provided the $R_{p}$ coupling is large enough. As a matter of fact in the pair production study, the sensitivity on the $R_{p}$ couplings is mainly provided by the displaced vertex analysis of the Lightest Supersymmetric Particle (LSP) decay which is difficult experimentally, especially at hadronic colliders. 
Neither the Grand Unified Theories (GUT), the string theories nor the study of the discrete gauge symmetries give a strong theoretical argument in favor of the R-parity violating or R-parity conserving scenarios [3]. Hence, the resonant production of SUSY particle through $R_{p}$ couplings is an attractive possibility which must be considered in the phenomenology of supersymmetry.

The hadronic colliders have an advantage in detecting new particles resonance. Indeed, due to the wide energy distribution of the colliding partons, the resonance can be probed in a wide range of the new particle mass. This is in contrast with the leptonic colliders for which the center of mass energy must be fine-tuned in order to discover new narrow width resonances.

At hadronic colliders, either a slepton or a squark can be produced at the resonance respectively through a $\lambda^{\prime}$ or a $\lambda^{\prime \prime}$ coupling constant. In the hypothesis of a single dominant $\not R$ coupling constant, the resonant scalar particle can decay through the same $R_{p}$ coupling as in the production, leading to a two quark final state for the hard process $[8,9,10,11,12]$. In the case where both $\lambda^{\prime}$ and $\lambda$ couplings are non-vanishing, the slepton produced via $\lambda^{\prime}$ can decay through $\lambda$ giving rise to the same final state as in Drell-Yan process, namely two leptons $[11,13,15]$. However, for reasonable values of the $R_{p}$ coupling constants, the decays of the resonant scalar particle via gauge interactions are typically dominant if kinematically allowed $[6,16]$.

The main decay of the resonant scalar particle through gauge interactions is the decay into its Standard Model partner plus a gaugino. Indeed, in the case where the resonant scalar particle is a squark, it is produced through $\lambda^{\prime \prime}$ interactions so that it must be a Right squark $\tilde{q}_{R}$ and thus it cannot decay into the $W^{ \pm}$-boson, which is the only other possible decay channel via gauge interactions. Besides, in the case where the resonant scalar particle is a slepton, it is a Left slepton produced via a $\lambda^{\prime}$ coupling but it cannot generally decay as $\tilde{l}_{L}^{ \pm} \rightarrow W^{ \pm} \tilde{\nu}_{L}$ or as $\tilde{\nu}_{L} \rightarrow W^{ \pm} \tilde{l}_{L}^{\mp}$. The reason is that in most of the SUSY models, as for example the supergravity or the gauge mediated models, the mass difference between the Left charged slepton and the Left sneutrino is due to the D-terms so that it is fixed by the relation $m_{\tilde{l}_{L}^{ \pm}}^{2}-m_{\tilde{\nu}_{L}}^{2}=\cos 2 \beta M_{W}^{2}[17]$ and thus it does not exceed the $W^{ \pm}$-boson mass. Nevertheless, we note that in the large $\tan \beta$ scenario, a resonant scalar particle of the third generation can generally decay into the $W^{ \pm}$-boson due to the large mixing in the third family sfermion sector. For instance, in the SUGRA model with a large $\tan \beta$ a tau-sneutrino produced at the resonance can decay as $\tilde{\nu}_{\tau} \rightarrow W^{ \pm} \tilde{\tau}_{1}^{\mp}, \tilde{\tau}_{1}^{\mp}$ being the lightest stau.

The resonant scalar particle production at hadronic colliders leads thus mainly to the single gaugino production, in case where the decay of the relevant scalar particle into gaugino is kinematically allowed. In this paper, we study the single gaugino productions at Tevatron Run II. The single gaugino productions at hadronic colliders were first studied in $[2,7]$. Later, studies on the single neutralino [18] and single chargino [19] productions at Tevatron have been performed. The single neutralino [20] and single chargino [21] productions have also been considered in the context of physics at LHC. In the present article, we also study the single superpartner productions at Tevatron Run II which occur via $2 \rightarrow 2-$ body processes and do not receive contributions from resonant SUSY particle productions.

The singly produced superpartner initiates a cascade decay ended typically by the $R_{p}$ decay of the LSP. In case of a single dominant $\lambda^{\prime \prime}$ coupling constant, the LSP decays into quarks so that this cascade decay leads to multijet final states having a large QCD background $[7,8]$. Nevertheless, if some leptonic decays, as for instance $\tilde{\chi}^{ \pm} \rightarrow l^{ \pm} \nu \tilde{\chi}^{0}$, 
$\tilde{\chi}^{ \pm}$being the chargino and $\tilde{\chi}^{0}$ the neutralino, enter the chain reaction, clearer leptonic signatures can be investigated [22]. In contrast, in the hypothesis of a single dominant $\lambda^{\prime}$ coupling constant, the LSP decay into charged leptons naturally favors leptonic signatures [2]. We will thus study the single superpartner production reaction at Tevatron Run II within the scenario of a single dominant $\lambda_{i j k}^{\prime}$ coupling constant.

In section 2 , we define our theoretical framework. In section 3 , we present the values of the cross sections for the various single superpartner productions via $\lambda_{i j k}^{\prime}$ at Tevatron Run II and we discuss the interesting multileptonic signatures that these processes can generate. In section 4, we analyse the three lepton signature induced by the single chargino production. In section 5, we study the like sign dilepton final state generated by the single neutralino and chargino productions.

\section{Theoretical framework}

Our framework throughout this paper will be the so-called minimal supergravity model (mSUGRA) which assumes the existence of a grand unified gauge theory and family universal boundary conditions on the supersymmetry breaking parameters. We choose the 5 following parameters: $m_{0}$ the universal scalars mass at the unification scale $M_{X}$, $m_{1 / 2}$ the universal gauginos mass at $M_{X}, A=A_{t}=A_{b}=A_{\tau}$ the trilinear Yukawa coupling at $M_{X}, \operatorname{sign}(\mu)$ the sign of the $\mu(t)$ parameter $\left(t=\log \left(M_{X}^{2} / Q^{2}\right), Q\right.$ denoting the running scale) and $\tan \beta=<H_{u}>/<H_{d}>$ where $\left\langle H_{u}>\right.$ and $\left\langle H_{d}>\right.$ denote the vacuum expectation values of the Higgs fields. In this model, the higgsino mixing parameter $|\mu|$ is determined by the radiative electroweak symmetry breaking condition. Note also that the parameters $m_{1 / 2}$ and $M_{2}(t)$ ( $\tilde{W}$ wino mass) are related by the solution of the one loop renormalization group equations $m_{1 / 2}=\left(1-\beta_{a} t\right) M_{a}(t)$ with $\beta_{a}=g_{X}^{2} b_{a} /(4 \pi)^{2}$, where $\beta_{a}$ are the beta functions, $g_{X}$ is the coupling constant at $M_{X}$ and $b_{a}=[3,-1,-11$, $a=[3,2,1]$ corresponding to the gauge group factors $S U(3)_{c}, S U(2)_{L}, S U(1)_{Y}$. We shall set the unification scale at $M_{X}=210^{16} \mathrm{GeV}$ and the running scale at the $Z^{0}$-boson mass: $Q=m_{Z^{0}}$.

We also assume the infrared fixed point hypothesis for the top quark Yukawa coupling [23] that provides a natural explanation of a large top quark mass $m_{t o p}$. In the infrared fixed point approach, $\tan \beta$ is fixed up to the ambiguity associated with large or low $\tan \beta$ solutions. The low solution of $\tan \beta$ is fixed by the equation $m_{\text {top }}=C \sin \beta$, where $C \approx 190-210 \mathrm{GeV}$ for $\alpha_{s}\left(m_{Z^{0}}\right)=0.11-0.13$. For instance, with a top quark mass of $m_{\text {top }}=174.2 \mathrm{GeV}$ [24], the low solution is given by $\tan \beta \approx 1.5$. The second important effect of the infrared fixed point hypothesis is that the dependence of the electroweak symmetry breaking constraint on the $A$ parameter becomes weak so that $|\mu|$ is a known function of the $m_{0}, m_{1 / 2}$ and $\tan \beta$ parameters [23].

Finally, we consider the $R_{p}$ extension of the mSUGRA model characterised by a single dominant $R_{p}$ coupling constant of type $\lambda_{i j k}^{\prime}$.

\section{Single superpartner productions via $\lambda_{i j k}^{\prime}$ at Tevatron Run II}

\subsection{Resonant superpartner production}

At hadronic colliders, either a sneutrino $(\tilde{\nu})$ or a charged slepton $(\tilde{l})$ can be produced at the resonance via the $\lambda_{i j k}^{\prime}$ coupling. As explained in Section 1, for most of the SUSY models, the slepton produced at the resonance has two possible gauge decays, namely a decay 

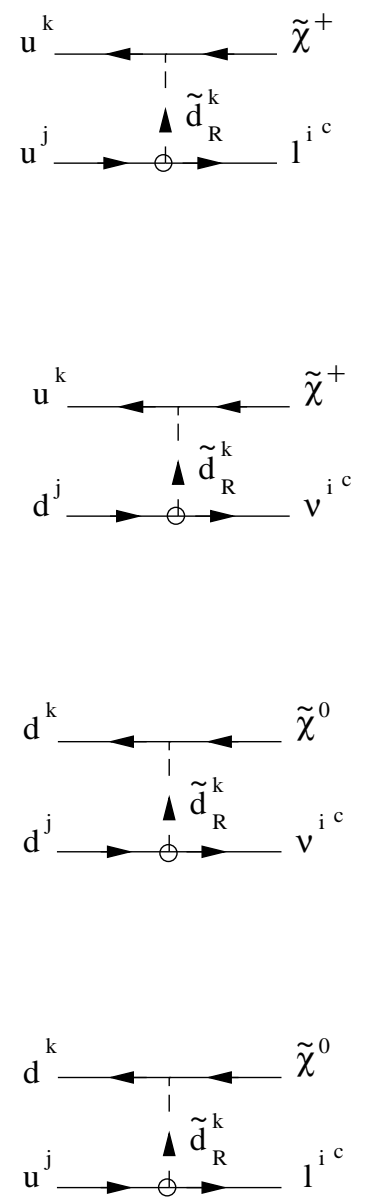

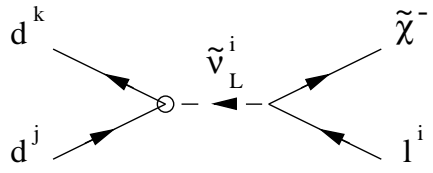

(a)
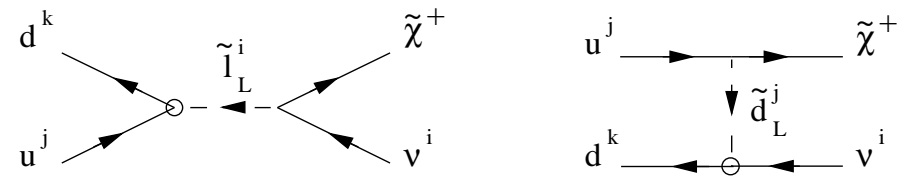

(b)
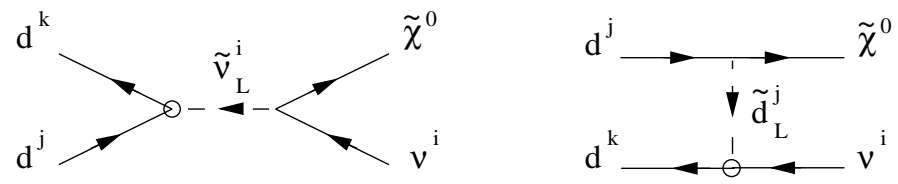

(c)
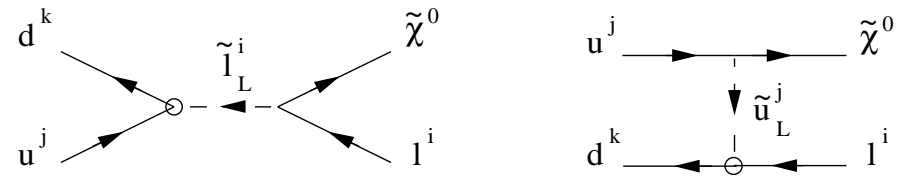

(d)

Figure 1: Feynman diagrams for the 4 single production reactions involving $\lambda_{i j k}^{\prime}$ at hadronic colliders which receive a contribution from a resonant supersymmetric particle production. The $\lambda_{i j k}^{\prime}$ coupling constant is symbolised by a small circle and the arrows denote the flow of the particle momentum.

into either a chargino or a neutralino. Therefore, in the scenario of a single dominant $\lambda_{i j k}^{\prime}$ coupling and for most of the SUSY models, either a chargino or a neutralino is singly produced together with either a charged lepton or a neutrino, through the resonant superpartner production at hadronic colliders. There are thus four main possible types of single superpartner production reaction involving $\lambda_{i j k}^{\prime}$ at hadronic colliders which receive a contribution from resonant SUSY particle production. The diagrams associated to these four reactions are drawn in Fig.1. As can be seen in this figure, these single superpartner productions receive also some contributions from both the $t$ and $u$ channels. Note that all the single superpartner production processes drawn in Fig.1 have charge conjugated processes. We have calculated the amplitudes of the processes shown in Fig.1 and the results are given in Appendix A.

\subsubsection{Cross sections}

In this section, we discuss the dependence of the single gaugino production cross sections on the various supersymmetric parameters. We will not assume here the radiative elec- 


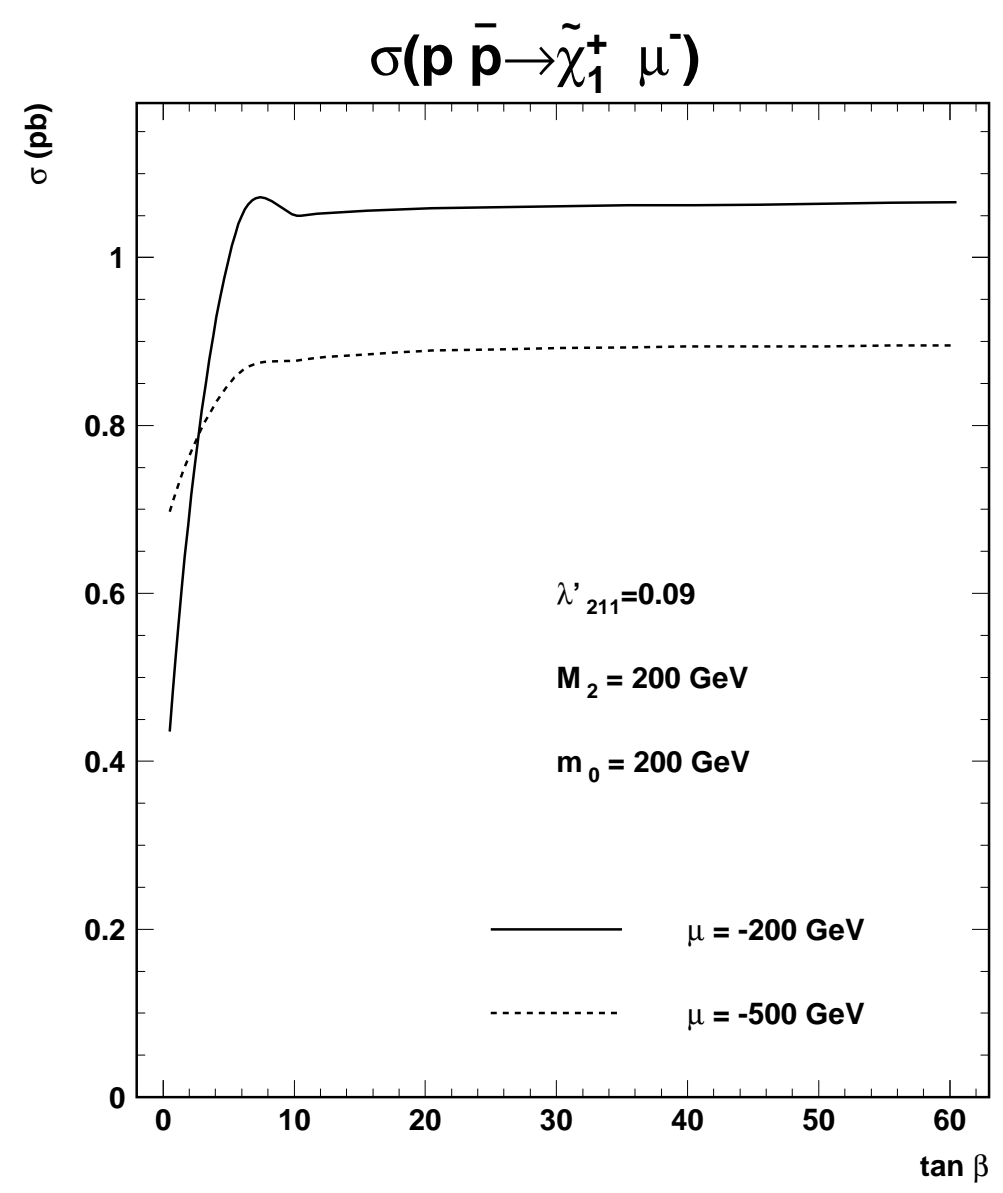

Figure 2: Cross sections (in pb) of the single chargino production $p \bar{p} \rightarrow \tilde{\chi}_{1}^{+} \mu^{-}$at a center of mass energy of $2 T e V$ as a function of the $\tan \beta$ parameter for $\lambda_{211}^{\prime}=0.09, M_{2}=200 \mathrm{GeV}, m_{0}=200 \mathrm{GeV}$ and two values of the $\mu$ parameter: $\mu=-200 \mathrm{GeV},-500 \mathrm{GeV}$.

troweak symmetry breaking condition in order to study the variations of the cross sections with the higgsino mixing parameter $\mu$.

First, we study the cross section of the single chargino production $p \bar{p} \rightarrow \tilde{\chi}^{+} l_{i}^{-}$which occurs through the $\lambda_{i j k}^{\prime}$ coupling (see Fig.1(a)). The differences between the $\tilde{\chi}^{+} e^{-}, \tilde{\chi}^{+} \mu^{-}$ and $\tilde{\chi}^{+} \tau^{-}$production (occuring respectively through the $\lambda_{1 j k}^{\prime}, \lambda_{2 j k}^{\prime}$ and $\lambda_{3 j k}^{\prime}$ couplings with identical $j$ and $k$ indices) cross sections involve $m_{l_{i}}$ lepton mass terms (see Appendix A) and are thus negligible. The $p \bar{p} \rightarrow \tilde{\chi}^{+} l_{i}^{-}$reaction receives contributions from the $s$ channel sneutrino exchange and the $t$ and $u$ channels squark exchanges as shown in Fig.1. However, the $t$ and $u$ channels represent small contributions to the whole single chargino production cross section when the sneutrino exchanged in the $s$ channel is real, namely for $m_{\tilde{\nu}_{i L}}>m_{\tilde{\chi}^{ \pm}}$. The $t$ and $u$ channels cross sections will be relevant only when the produced sneutrino is virtual since the $s$ channel contribution is small. In this situation the single chargino production rate is greatly reduced compared to the case where the exchanged sneutrino is produced as a resonance. Hence, The $t$ and $u$ channels do not represent important contributions to the $\tilde{\chi}^{+} l_{i}^{-}$production rate.

The dependence of the $\tilde{\chi}^{+} l_{i}^{-}$production rate on the $A$ coupling is weak. Indeed, the 


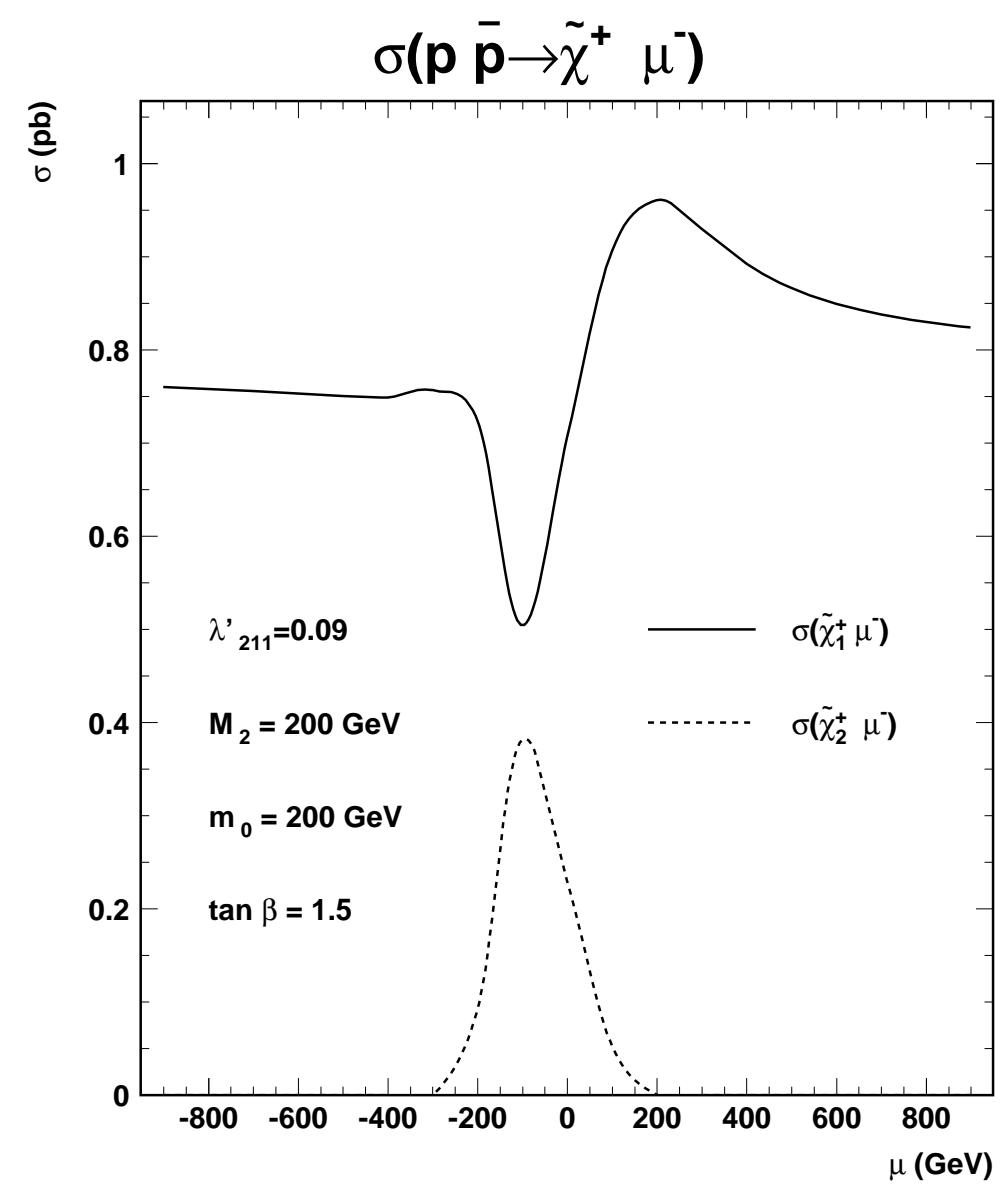

Figure 3: Cross sections (in pb) of the single chargino productions $p \bar{p} \rightarrow \tilde{\chi}_{1,2}^{+} \mu^{-}$as a function of the $\mu$ parameter (in $\mathrm{GeV}$ ) for $\lambda_{211}^{\prime}=0.09, M_{2}=200 \mathrm{GeV}, \tan \beta=1.5$ and $m_{0}=200 \mathrm{GeV}$ at a center of mass energy of $2 \mathrm{TeV}$.

rate depends on the $A$ parameter only through the masses of the third generation squarks eventually exchanged in the $t$ and $u$ channels (see Fig.1). Similarly, the dependences on the $A$ coupling of the rates of the other single gaugino productions shown in Fig.1 are weak. Therefore, in this article we present the results for $A=0$. Later, we will discuss the effects of large $A$ couplings on the cascade decays which are similar to the effects of large $\tan \beta$ values.

$\tan \beta$ dependence: The dependence of the $\tilde{\chi}^{+} l_{i}^{-}$production rate on $\tan \beta$ is also weak, except for $\tan \beta<10$. This can be seen in Fig. 2 where the cross section of the $p \bar{p} \rightarrow \tilde{\chi}_{1}^{+} \mu^{-}$ reaction occuring through the $\lambda_{211}^{\prime}$ coupling is shown as a function of the $\tan \beta$ parameter. The choice of the $\lambda_{211}^{\prime}$ coupling is motivated by the fact that the analysis in Sections 4 and 5 are explicitly made for this $R_{p}$ coupling. In Fig.2, we have taken the $\lambda_{211}^{\prime}$ value equal to its low-energy experimental bound for $m_{\tilde{d}_{R}}=100 \mathrm{GeV}$ which is $\lambda_{211}^{\prime}<0.09$ [4]. At this stage, some remarks on the values of the cross sections presented in this section must be done. First, the single gaugino production rates must be multiplied by a factor 2 in order to take into account the charge conjugated process, which is for example in the present case $p \bar{p} \rightarrow \tilde{\chi}^{-} \mu^{+}$. Furthermore, the values of the cross sections for all the single gaugino productions are obtained using the CTEQ4L structure function [25]. 


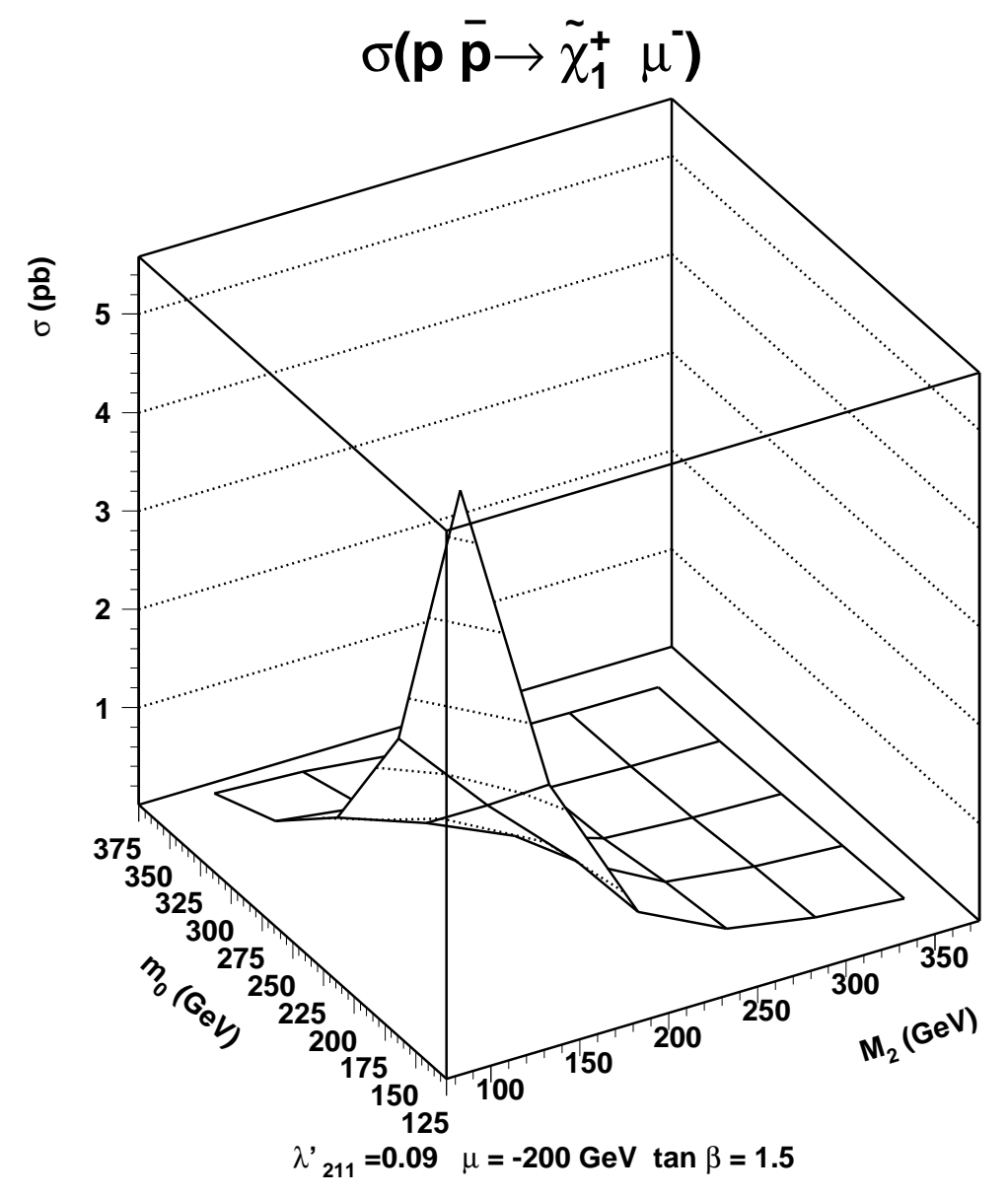

Figure 4: Cross section (in pb) of the single chargino production $p \bar{p} \rightarrow \tilde{\chi}_{1}^{+} \mu^{-}$as a function of the $m_{0}$ (in $G e V$ ) and $M_{2}$ (in $\mathrm{GeV}$ ) parameters. The center of mass energy is $\sqrt{s}=2 T e V$ and the other parameters are: $\lambda_{211}^{\prime}=0.09, \tan \beta=1.5$ and $\mu=-200 \mathrm{GeV}$.

Choosing other parametrizations does not change significantly the results since proton structure functions in our kinematical domain in Bjorken $x$ are known and have been already measured. For instance, with the set of parameters $\lambda_{211}^{\prime}=0.09, M_{2}=100 \mathrm{GeV}$, $\tan \beta=1.5, m_{0}=300 \mathrm{GeV}$ and $\mu=-500 \mathrm{GeV}$, the $\tilde{\chi}_{1}^{+} \mu^{-}$production cross section is $0.503 p b$ for the CTEQ4L structure function [25], 0.503pb for the BEP structure function [26], $0.480 p b$ for the MRS (R2) structure function [27] and 0.485pb for the GRV LO structure function [28].

$\mu$ dependence: In Fig.3, we present the cross sections of the $\tilde{\chi}_{1}^{+} \mu^{-}$and $\tilde{\chi}_{2}^{+} \mu^{-}$productions as a function of the $\mu$ parameter. We observe in this figure the weak dependence of the cross section $\sigma\left(p \bar{p} \rightarrow \tilde{\chi}_{1}^{+} \mu^{-}\right)$on $\mu$ for $|\mu|>M_{2}$. The reason is the smooth dependence of the $\tilde{\chi}_{1}^{ \pm}$mass on $\mu$ in this domain. However, the rate strongly decreases in the region $|\mu|<M_{2}$ in which the $\tilde{\chi}_{1}^{ \pm}$chargino is mainly composed by the higgsino. Nevertheless, the small $|\mu|$ domain $\left(|\mu|\right.$ smaller than $\sim 100 \mathrm{GeV}$ for $\tan \beta=1.41, M_{2}>100 \mathrm{GeV}$, $m_{0}=500 \mathrm{GeV}$ and $\left.\lambda^{\prime} \neq 0\right)$ is excluded by the present experimental limits derived from the LEP data [29].

In contrast, the cross section $\sigma\left(p \bar{p} \rightarrow \tilde{\chi}_{2}^{+} \mu^{-}\right)$increases in the domain $|\mu|<M_{2}$. The expla- 


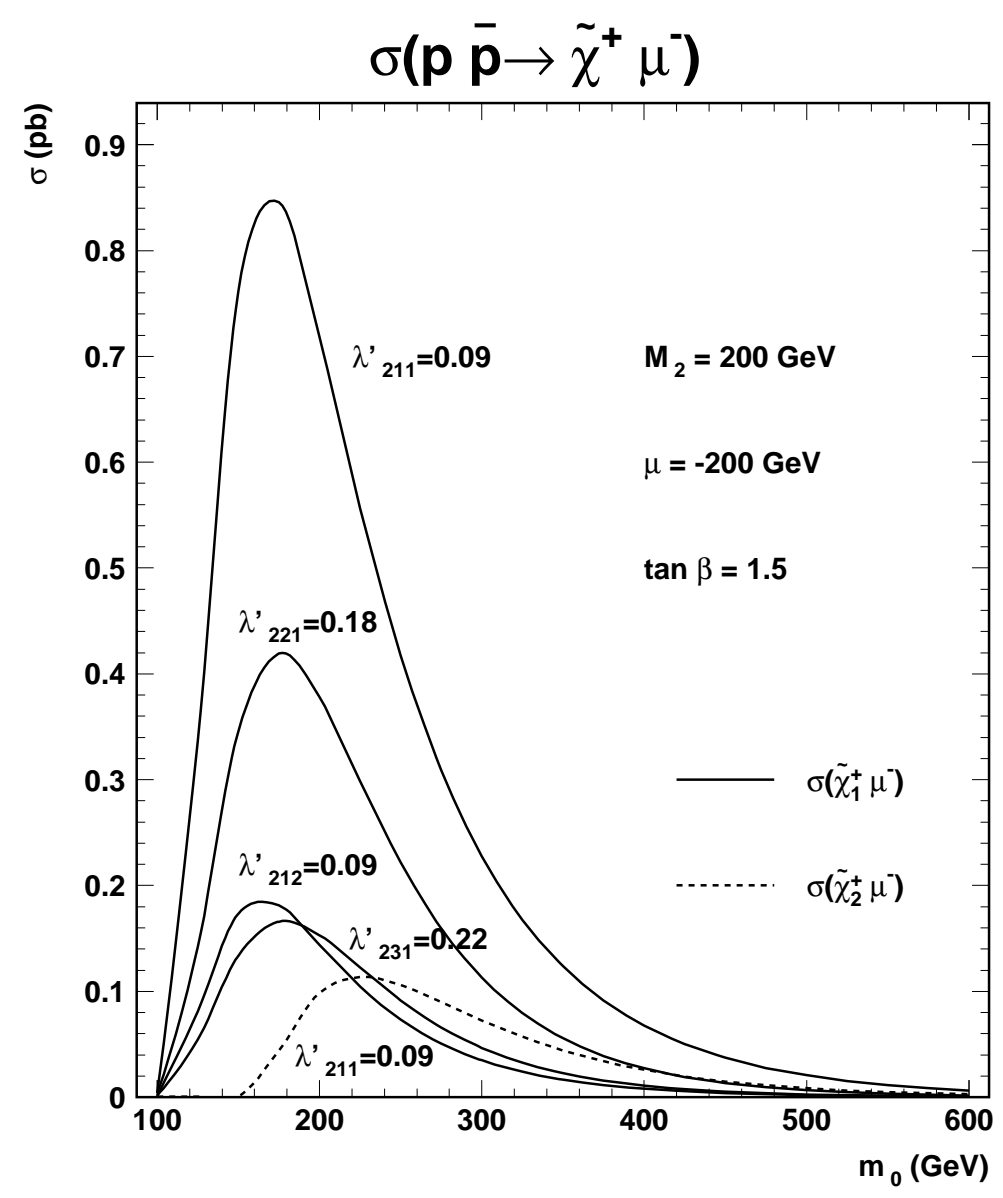

Figure 5: Cross sections (in $p b$ ) of the single chargino productions $p \bar{p} \rightarrow \tilde{\chi}_{1,2}^{+} \mu^{-}$as a function of the $m_{0}$ parameter (in $\mathrm{GeV})$. The center of mass energy is taken at $\sqrt{s}=2 \mathrm{TeV}$ and $\lambda_{211}^{\prime}=0.09, M_{2}=200 \mathrm{GeV}, \tan \beta=1.5$ and $\mu=-200 \mathrm{GeV}$. The rates of the single $\tilde{\chi}_{1}^{+}$production via the $\not R_{p}$ couplings $\lambda_{212}^{\prime}=0.09, \lambda_{221}^{\prime}=0.18$ and $\lambda_{231}^{\prime}=0.22$ are also shown. The chosen values of the $\not R_{p}$ couplings correspond to the low-energy limits [4] for a squark mass of $100 \mathrm{GeV}$.

nation is that the $\tilde{\chi}_{2}^{ \pm}$mass is enhanced as $|\mu|$ increases. The region in which $\sigma\left(p \bar{p} \rightarrow \tilde{\chi}_{2}^{+} \mu^{-}\right)$ becomes important is at small values of $|\mu|$, near the LEP limits of [29]. We also remark in Fig. 3 that the single $\tilde{\chi}_{1}^{+}$production rate values remain above the single $\tilde{\chi}_{2}^{+}$production rate values in all the considered range of $\mu$. In this figure, we also notice that the cross section is smaller when $\mu$ is negative. To be conservative, we will take $\mu<0$ in the following.

$m_{0}$ and $M_{2}$ dependences: In fact, the cross section $\sigma\left(p \bar{p} \rightarrow \tilde{\chi}^{+} l_{i}^{-}\right)$depends mainly on the $m_{0}$ and $M_{2}$ parameters. We present in Fig. 4 the rate of the $\tilde{\chi}_{1}^{+} \mu^{-}$production as a function of the $m_{0}$ and $M_{2}$ parameters. The rate decreases at high values of $m_{0}$ since the sneutrino becomes heavier as $m_{0}$ increases and more energetic initial partons are required in order to produce the resonant sneutrino. The decrease of the rate at large values of $M_{2}$ is due to the increase of the chargino mass and thus the reduction of the phase space factor.

In Fig.5, we show the variations of the $\sigma\left(p \bar{p} \rightarrow \tilde{\chi}_{1}^{+} \mu^{-}\right)$cross sections with $m_{0}$ for fixed values of $M_{2}, \mu$ and $\tan \beta$. The cross sections corresponding to the $\tilde{\chi}_{1}^{+} \mu^{-}$production 
through various $R_{p}$ couplings of type $\lambda_{2 j k}^{\prime}$ are presented. In this figure, we only consider the $R_{p}$ couplings giving the highest cross sections. The values of the considered $\lambda_{2 j k}^{\prime}$ couplings have been taken at their low-energy limit [4] for a squark mass of $100 \mathrm{GeV}$. The rate of the $\tilde{\chi}_{2}^{+} \mu^{-}$production through $\lambda_{211}^{\prime}$ is also shown in this figure. We already notice that the cross section is significant for many $R_{p}$ couplings and we will come back on this important statement in the following.

The $\sigma\left(p \bar{p} \rightarrow \tilde{\chi}^{+} \mu^{-}\right)$rates decrease as $m_{0}$ increases for the same reason as in Fig.4. A decrease of the rates also occurs at small values of $m_{0}$. The reason is the following. When $m_{0}$ decreases, the $\tilde{\nu}$ mass is getting closer to the $\tilde{\chi}^{ \pm}$masses so that the phase space factor associated to the decay $\tilde{\nu}_{\mu} \rightarrow \tilde{\chi}^{ \pm} \mu^{\mp}$ decreases.

We also observe that the single $\tilde{\chi}_{2}^{+}$production rate is much smaller than the single $\tilde{\chi}_{1}^{+}$ production rate, as in Fig.3.

The differences between the $\tilde{\chi}_{1}^{+} \mu^{-}$production rates occuring via the various $\lambda_{2 j k}^{\prime}$ couplings are explained by the different parton densities. Indeed, as shown in Fig.1 the hard process associated to the $\tilde{\chi}_{1}^{+} \mu^{-}$production occuring through the $\lambda_{2 j k}^{\prime}$ coupling constant has a partonic initial state $\bar{q}_{j} q_{k}$. The $\tilde{\chi}_{1}^{+} \mu^{-}$production via the $\lambda_{211}^{\prime}$ coupling has first generation quarks in the initial state which provide the maximum parton density.

We now discuss the rate behaviours for the reactions $p \bar{p} \rightarrow \tilde{\chi}^{-} \nu_{\mu}, p \bar{p} \rightarrow \tilde{\chi}^{0} \mu^{-}$and $p \bar{p} \rightarrow \tilde{\chi}^{0} \nu_{\mu}$ which occur via $\lambda_{211}^{\prime}$, in the SUSY parameter space. The dependences of these rates on the $A, \tan \beta, \mu$ and $M_{2}$ parameters are typically the same as for the $\tilde{\chi}^{+} \mu^{-}$ production rate. The variations of the $\tilde{\chi}_{1}^{-} \nu_{\mu}, \tilde{\chi}_{1,2}^{0} \mu^{-}$and $\tilde{\chi}_{1}^{0} \nu_{\mu}$ productions cross sections with the $m_{0}$ parameter are shown in Fig.6. The $\tilde{\chi}_{2}^{-} \nu_{\mu}, \tilde{\chi}_{3,4}^{0} \mu^{-}$and $\tilde{\chi}_{3,4}^{0} \nu_{\mu}$ production rates are comparatively negligible and thus have not been represented. We observe in this figure that the cross sections decrease at large $m_{0}$ values like the $\tilde{\chi}^{+} \mu^{-}$production rate. However, while the single $\tilde{\chi}_{1}^{ \pm}$productions rates decrease at small $m_{0}$ values (see Fig.5 and Fig.6), this is not true for the single $\tilde{\chi}_{1}^{0}$ productions (see Fig.6). The reason is that in mSUGRA the $\tilde{\chi}_{1}^{0}$ and $\tilde{l}_{i L}\left(l_{i}=l_{i}^{ \pm}, \nu_{i}\right)$ masses are never close enough to induce a significant decrease of the cross section associated to the reaction $p \bar{p} \rightarrow \tilde{l}_{i L} \rightarrow \tilde{\chi}_{1}^{0} l_{i}$, where $l_{i}=l_{i}^{ \pm}, \nu_{i}$ (see Fig.1(c)(d)), caused by a phase space factor reduction. Therefore, the resonant slepton contribution to the single $\tilde{\chi}_{1}^{0}$ production is not reduced at small $m_{0}$ values like the resonant slepton contribution to the single $\tilde{\chi}_{1}^{ \pm}$production. For the same reason, the single $\tilde{\chi}_{1}^{0}$ productions have much higher cross sections than the single $\tilde{\chi}_{1}^{ \pm}$ productions in most of the mSUGRA parameter space, as illustrate Fig.5 and Fig.6. We note that in the particular case of a single dominant $\lambda_{3 j k}^{\prime}$ coupling constant and of large $\tan \beta$ values, the rate of the reaction $p \bar{p} \rightarrow \tilde{\tau}_{1}^{ \pm} \rightarrow \tilde{\chi}_{1}^{0} \tau^{ \pm}$(see Fig. $1(\mathrm{~d})$ ), where $\tilde{\tau}_{1}^{ \pm}$is the lightest tau-slepton, can be reduced at low $m_{0}$ values since then $m_{\tilde{\tau}_{1}^{ \pm}}$can be closed to $m_{\tilde{\chi}_{1}^{0}}$ due to the large mixing occuring in the staus sector. By analysing Fig.5 and Fig.6, we also remark that the $\tilde{\chi}^{-} \nu_{\mu}\left(\tilde{\chi}^{0} \mu^{-}\right)$production rate is larger than the $\tilde{\chi}^{+} \mu^{-}\left(\tilde{\chi}^{0} \nu_{\mu}\right)$ one. The explanation is that in $p \bar{p}$ collisions the initial states of the resonant charged slepton production $u_{j} \bar{d}_{k}, \bar{u}_{j} d_{k}$ have higher partonic densities than the initial states of the resonant sneutrino production $d_{j} \bar{d}_{k}, \bar{d}_{j} d_{k}$. This phenomenon also increases the difference between the rates of the $\tilde{\chi}_{1}^{0} \mu^{-}$and $\tilde{\chi}_{1}^{+} \mu^{-}$productions at Tevatron.

Although the single $\tilde{\chi}_{1}^{ \pm}$production cross sections are smaller than the $\tilde{\chi}_{1}^{0}$ ones, it is interesting to study both of them since they have quite high values. 


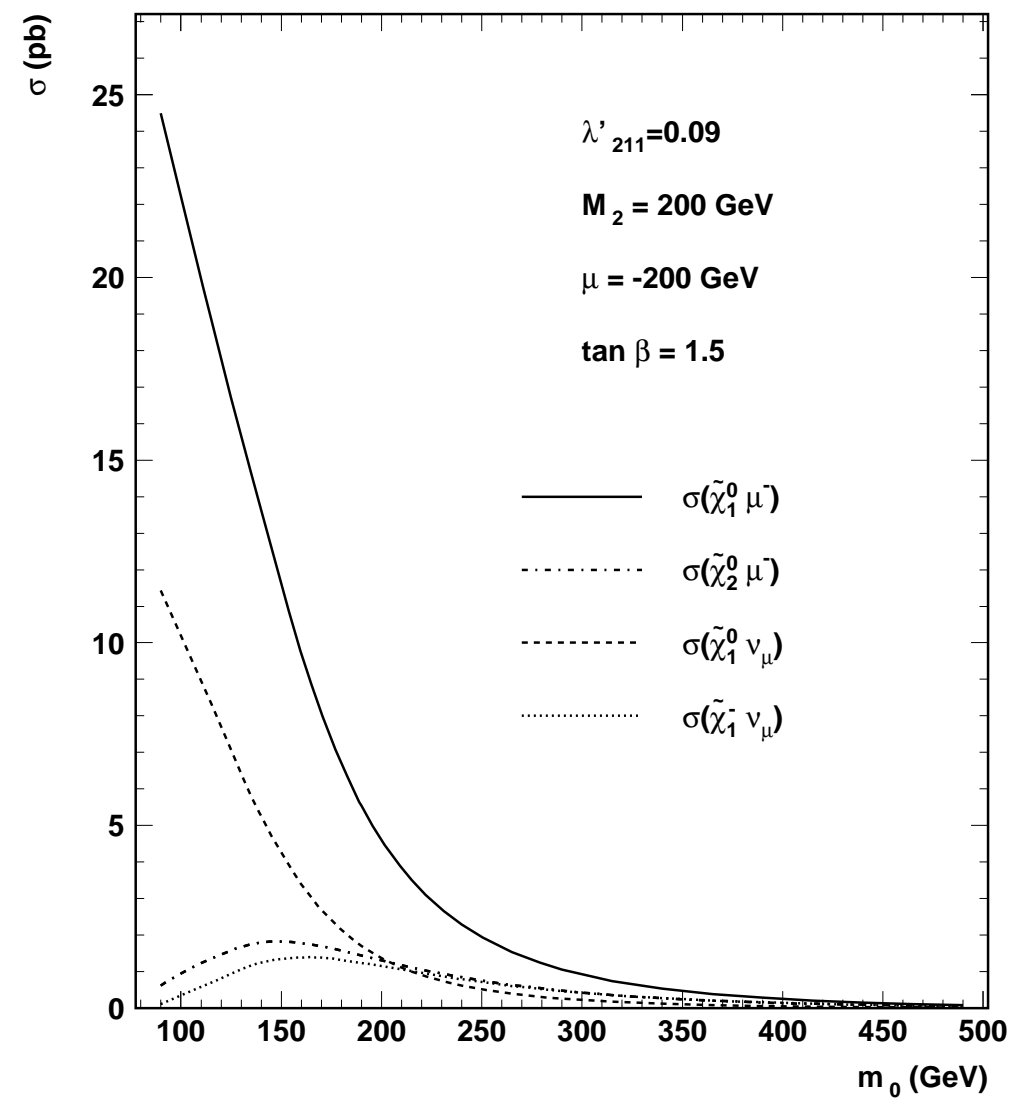

Figure 6: Cross sections (in $p b$ ) of the reactions $p \bar{p} \rightarrow \tilde{\chi}_{1}^{-} \nu, p \bar{p} \rightarrow \tilde{\chi}_{1,2}^{0} \mu^{-}$and $p \bar{p} \rightarrow \tilde{\chi}_{1}^{0} \nu$ as a function of the $m_{0}$ parameter (in $\mathrm{GeV}$ ). The center of mass energy is taken at $\sqrt{s}=2 \mathrm{TeV}$ and the considered set of parameters is: $\lambda_{211}^{\prime}=0.09$, $M_{2}=200 G e V, \tan \beta=1.5$ and $\mu=-200 G e V$.

\subsection{Non-resonant superpartner production}

At hadronic colliders, the single productions of SUSY particle via $\lambda_{i j k}^{\prime}$ can occur through some $2 \rightarrow 2-$ body processes which do not receive contributions from any resonant superpartner production. These non-resonant superpartner productions are (one must also add the charge conjugated processes):

- The gluino production $\bar{u}_{j} d_{k} \rightarrow \tilde{g} l_{i}$ via the exchange of a $\tilde{u}_{j L}\left(\tilde{d}_{k R}\right)$ squark in the $t(u)$ channel.

- The squark production $\bar{d}_{j} g \rightarrow \tilde{d}_{k R}^{*} \nu_{i}$ via the exchange of a $\tilde{d}_{k R}$ squark (d $d_{j}$ quark) in the $t(s)$ channel.

- The squark production $\bar{u}_{j} g \rightarrow \tilde{d}_{k R}^{*} l_{i}$ via the exchange of a $\tilde{d}_{k R}$ squark ( $u_{j}$ quark) in the $t(s)$ channel.

- The squark production $d_{k} g \rightarrow \tilde{d}_{j L} \nu_{i}$ via the exchange of a $\tilde{d}_{j L}$ squark ( $d_{k}$ quark) in the $t(s)$ channel. 
- The squark production $d_{k} g \rightarrow \tilde{u}_{j L} l_{i}$ via the exchange of a $\tilde{u}_{j L}$ squark ( $d_{k}$ quark) in the $t(s)$ channel.

- The sneutrino production $\bar{d}_{j} d_{k} \rightarrow Z \tilde{\nu}_{i L}$ via the exchange of a $d_{k}$ or $d_{j}$ quark $\left(\tilde{\nu}_{i L}\right.$ sneutrino) in the $t(s)$ channel.

- The charged slepton production $\bar{u}_{j} d_{k} \rightarrow Z \tilde{l}_{i L}$ via the exchange of a $d_{k}$ or $u_{j}$ quark $\left(\tilde{l}_{i L}\right.$ slepton) in the $t(s)$ channel.

- The sneutrino production $\bar{u}_{j} d_{k} \rightarrow W^{-} \tilde{\nu}_{i L}$ via the exchange of a $d_{j}$ quark $\left(\tilde{l}_{i L}\right.$ sneutrino) in the $t(s)$ channel.

- The charged slepton production $\bar{d}_{j} d_{k} \rightarrow W^{+} \tilde{l}_{i L}$ via the exchange of a $u_{j}$ quark $\left(\tilde{\nu}_{i L}\right.$ sneutrino) in the $t(s)$ channel.

The single gluino production cannot reach high cross sections due to the strong experimental limits on the squarks and gluinos masses which are typically about $m_{\tilde{q}}, m_{\tilde{g}} \gtrsim$ $200 \mathrm{GeV}$ [30]. Indeed, the single gluino production occurs through the exchange of squarks in the $t$ and $u$ channels, as described above, so that the cross section of this production decreases as the squarks and gluinos masses increase. For the value $m_{\tilde{q}}=m_{\tilde{g}}=250 \mathrm{GeV}$ which is close to the experimental limits, we find the single gluino production rate $\sigma(p \bar{p} \rightarrow \tilde{g} \mu) \approx 10^{-2} p b$ which is consistent with the results of [7]. The cross sections given in this section are computed at a center of mass energy of $\sqrt{s}=2 \mathrm{TeV}$ using the version 33.18 of the COMPHEP routine [31] with the CTEQ4m structure function and an $R_{p}$ coupling $\lambda_{211}^{\prime}=0.09$. Similarly, the single squark production cross section cannot be large: for $m_{\tilde{q}}=250 \mathrm{GeV}$, the rate $\sigma\left(p \bar{p} \rightarrow \tilde{u}_{L} \mu\right)$ is of order $\sim 10^{-3} p b$. The production of a slepton together with a massive gauge boson has a small phase space factor and does not involve strong interaction couplings. The cross section of this type of reaction is thus small. For instance, with a slepton mass of $m_{\tilde{l}}=100 \mathrm{GeV}$ we find the cross section $\sigma\left(p \bar{p} \rightarrow Z \tilde{\mu}_{L}\right)$ to be of order $10^{-2} p b$.

As a conclusion, the non-resonant single superpartner productions have small rates and will not be considered here. Nevertheless, some of these reactions are interesting as their cross section involves few SUSY parameters, namely only one scalar superpartner mass and one $R_{p}$ coupling constant.

\section{Three lepton signature analysis}

\subsection{Signal}

In this section, we study the three lepton signature at Tevatron Run II generated by the single chargino production through $\lambda_{i j k}^{\prime}, p \bar{p} \rightarrow \tilde{\chi}^{ \pm} l_{i}^{\mp}$, followed by the cascade decay, $\tilde{\chi}^{ \pm} \rightarrow \tilde{\chi}_{1}^{0} l^{ \pm} \nu, \tilde{\chi}_{1}^{0} \rightarrow l_{i} u_{j} \bar{d}_{k}, \bar{l}_{i} \bar{u}_{j} d_{k}$ (the indices $i, j, k$ correspond to the indices of $\lambda_{i j k}^{\prime}$ ). In fact, the whole final state is 3 charged leptons +2 hard jets + missing energy $(\not E)$. The two jets and the missing energy come respectively from the quarks and the neutrino produced in the cascade decay. In the mSUGRA model, which predicts the $\tilde{\chi}_{1}^{0}$ as the LSP in most of the parameter space, the $p \bar{p} \rightarrow \tilde{\chi}^{ \pm} l_{i}^{\mp}$ reaction is the only single gaugino production allowing the three lepton signature to be generated in a significant way. Since the $\tilde{\chi}_{1}^{ \pm} l_{i}^{\mp}$ production rate is dominant compared to the $\tilde{\chi}_{2}^{ \pm} l_{i}^{\mp}$ production rate, as discussed in Section 3.1.1, we only consider the contribution to the three lepton signature from the single lightest chargino production. 
For $m_{\tilde{\nu}}, m_{\tilde{l}}, m_{\tilde{q}}, m_{\tilde{\chi}_{2}^{0}}>m_{\tilde{\chi}_{1}^{ \pm}}$, the branching ratio $B\left(\tilde{\chi}_{1}^{ \pm} \rightarrow \tilde{\chi}_{1}^{0} l^{ \pm} \nu\right)$ is typically of order $30 \%$ and is smaller than for the other possible decay $\tilde{\chi}_{1}^{ \pm} \rightarrow \tilde{\chi}_{1}^{0} \bar{q}_{p} q_{p}^{\prime}$ because of the color factor.

Since in our framework the $\tilde{\chi}_{1}^{0}$ is the LSP, it can only decay via $\lambda_{i j k}^{\prime}$, either as $\tilde{\chi}_{1}^{0} \rightarrow l_{i} u_{j} d_{k}$ or as $\tilde{\chi}_{1}^{0} \rightarrow \nu_{i} d_{j} d_{k}$, with a branching ratio $B\left(\tilde{\chi}_{1}^{0} \rightarrow l_{i} u_{j} d_{k}\right)$ ranging between $\sim 40 \%$ and $\sim 70 \%$.

The three lepton signature is particularly attractive at hadronic colliders because of the possibility to reduce the associated Standard Model background. In Section 4.2 we describe this Standard Model background and in Section 4.4 we show how it can be reduced.

\subsection{Standard Model background of the 3 lepton signature at Tevatron}

The first source of Standard Model background for the three leptons final state is the top quark pair production $q \bar{q} \rightarrow t \bar{t}$ or $g g \rightarrow t \bar{t}$. Since the top quark life time is smaller than its hadronisation time, the top decays and its main channel is the decay into a $W$ gauge boson and a bottom quark as $t \rightarrow b W$. The $t \bar{t}$ production can thus give rise to a $3 l$ final state if the $W$ bosons and one of the b-quarks undergo leptonic decays simultaneously. The cross section, calculated at leading order with PYTHIA [32] using the CTEQ2L structure function, times the branching fraction is $\sigma(p \bar{p} \rightarrow t \bar{t}) \times B^{2}\left(W \rightarrow l_{p} \nu_{p}\right) \approx 863 \mathrm{fb}(704 \mathrm{fb})$ with $p=1,2,3$ at $\sqrt{s}=2 \mathrm{TeV}$ for a top quark mass of $m_{\text {top }}=170 \mathrm{GeV}(175 \mathrm{GeV})$.

The other major source of Standard Model background is the $W^{ \pm} Z^{0}$ production followed by the leptonic decays of the gauge bosons, namely $W \rightarrow l \nu$ and $Z \rightarrow l \bar{l}$. The value for the cross section times the branching ratios is $\sigma(p \bar{p} \rightarrow W Z) \times B\left(W \rightarrow l_{p} \nu_{p}\right) \times B(Z \rightarrow$ $\left.l_{p} \bar{l}_{p}\right) \approx 82 f b(p=1,2,3)$ at leading order with a center of mass energy of $\sqrt{s}=2 \mathrm{TeV}$.

The $W^{ \pm} Z^{0}$ production gives also a small contribution to the 3 leptons background through the decays: $W \rightarrow b u_{p}$ and $Z \rightarrow b \bar{b}, W \rightarrow l \nu$ and $Z \rightarrow b \bar{b}$ or $W \rightarrow b u_{p}$ and $Z \rightarrow l \bar{l}$, if a lepton is produced in each of the $b$ jets.

Similarly, the $Z^{0} Z^{0}$ production followed by the decays $Z \rightarrow l \bar{l}(l=e, \mu), Z \rightarrow \tau \bar{\tau}$, where one of the $\tau$ decays into lepton while the other decays into jet, leads to three leptons in the final state. Within the same framework as above, the cross section is of order $\sigma(p \bar{p} \rightarrow Z Z \rightarrow 3 l) \approx 2 f b$.

The $Z^{0} Z^{0}$ production can also contribute weakly to the 3 leptons background via the decays: $Z \rightarrow l \bar{l}$ and $Z \rightarrow b \bar{b}$ or $Z \rightarrow b \bar{b}$ and $Z \rightarrow b \bar{b}$, since a lepton can be produced in a $\mathrm{b}$ jet.

It has been pointed out recently that the $W Z^{*}$ (throughout this paper a star indicates a virtual particle) and the $W \gamma^{*}$ productions could represent important contributions to the trilepton background $[33,34]$. The complete list of contributions to the 3 leptons final state from the $W Z, W \gamma^{*}$ and $Z Z$ productions, including cases where either one or both of the gauge bosons can be virtual, has been calculated in [35]. The authors of [35] have found that the $W Z, W \gamma^{*}$ and $Z Z$ backgrounds (including virtual boson(s)) at the upgraded Tevatron have together a cross section of order $0.5 \mathrm{fb}$ after the following cuts have been implemented: $P_{t}\left(l_{1}\right)>20 \mathrm{GeV}, P_{t}\left(l_{2}\right)>15 \mathrm{GeV}, P_{t}\left(l_{3}\right)>10 \mathrm{GeV} ;\left|\eta\left(l_{1}, l_{2,3}\right)\right|<1.0,2.0$; $I S O_{\delta R=0.4}<2 \mathrm{GeV} ; H_{T}>25 \mathrm{GeV} ; 81 \mathrm{GeV}<M_{i n v}(l \bar{l})<101 \mathrm{GeV} ; 12 \mathrm{GeV}<M_{i n v}(l \bar{l})$; $60 \mathrm{GeV}<m_{T}\left(l, E_{T}\right)<85 \mathrm{GeV}$.

We note that there is at most one hard jet in the 3 leptons backgrounds generated by the $W Z, W \gamma *$ and $Z Z$ productions (including virtual boson(s)). Since the number of hard jets is equal to 2 in our signal (see Section 4.1), a jet veto can thus reduce this Standard 


\begin{tabular}{|c|c|c|c|c|c|}
\hline$m_{1 / 2} \backslash m_{0}$ & $100 \mathrm{GeV}$ & $200 \mathrm{GeV}$ & $300 \mathrm{GeV}$ & $400 \mathrm{GeV}$ & $500 \mathrm{GeV}$ \\
\hline $100 \mathrm{GeV}$ & 5.775 & 3.376 & 2.849 & 2.974 & 3.195 \\
\hline $200 \mathrm{GeV}$ & 0.147 & 0.122 & 0.123 & 0.130 & 0.138 \\
\hline $300 \mathrm{GeV}$ & $1.810^{-2}$ & $1.310^{-2}$ & $1.210^{-2}$ & $1.310^{-2}$ & $1.310^{-2}$ \\
\hline
\end{tabular}

Table 1: Cross section (in $p b$ ) of the sum of all the superpartners pair productions at Tevatron Run II as a function of the $m_{0}$ and $m_{1 / 2}$ parameters for $\tan \beta=1.5, \operatorname{sign}(\mu)<0$ and $\lambda_{211}^{\prime}=0.05$ at a center of mass energy of $\sqrt{s}=2 \mathrm{TeV}$. These rates have been calculated with HERWIG [44] using the EHLQ2 structure function.

Model background with respect to the signal.

Other small sources of Standard Model background have been estimated in [36]: The productions like $Z b, W t$ or $W t \bar{t}$. After applying cuts on the geometrical acceptance, the transverse momentum and the isolation, these backgrounds are expected to be at most of order $10^{-4} p b$ in $p \bar{p}$ collisions with a center of mass energy of $\sqrt{s}=2 \mathrm{TeV}$. We have checked that the $Z b$ production gives a negligible contribution to the 3 lepton signature.

There are finally some non-physics sources of background. First, the 4 leptons signal, which can be generated by the $Z^{0} Z^{0}$ and $t \bar{t}$ productions, appears as a 3 leptons signature if one of the leptons is missed. Besides, the processes $p \bar{p} \rightarrow Z+X$, Drell $-Y$ an $+X$ would mimic a trilepton signal if $X$ fakes a lepton. Monte Carlo simulations using simplified detector simulation, like for example SHW [37] as in the present study (see Section 4.4), cannot give a reliable estimate of this background. A knowledge of the details of the detector response as well as the jet fragmentation is necessary in order to determinate the probability to fake a lepton. In [38], using standard cuts the background coming from $p \bar{p} \rightarrow Z+X$, Drell - Yan $+X$ has been estimated to be of order $2 f b$ at Tevatron with $\sqrt{s}=2 \mathrm{TeV}$. The authors of [38] have also estimated the background from the three-jet events faking trilepton signals to be around $10^{-3} \mathrm{fb}$.

Hence for the study of the Standard Model background associated to the 3 lepton signature at Tevatron Run II, we consider the $W^{ \pm} Z^{0}$ production and both the physics and non-physics contributions generated by the $Z^{0} Z^{0}$ and $t \bar{t}$ productions.

\subsection{Supersymmetric background of the 3 lepton signature at Tevatron}

If an excess of events is observed in the three lepton channel at Tevatron, one would wonder what is the origin of those anomalous events. One would thus have to consider all of the supersymmetric productions leading to the three lepton signature. In the present context of R-parity violation, multileptonic final states can be generated by the single chargino production involving $R_{p}$ couplings, but also by the supersymmetric particle pair production which involves only gauge couplings [39, 40]. In $R_{p}$ models, the superpartner pair production can even lead to the trilepton signature [41, 42, 43]. As a matter of fact, both of the produced supersymmetric particles decay, either directly or through cascade decays, into the LSP which is the neutralino in our framework. In the hypothesis of a dominant $\lambda^{\prime}$ coupling constant, each of the 2 produced neutralinos can decay into a charged lepton and two quarks: at least two charged leptons and four jets in the final state are produced. The third charged lepton can be generated in the cascade decays as for example at the level of the chargino decay $\tilde{\chi}^{ \pm} \rightarrow \tilde{\chi}^{0} l^{ \pm} \nu$.

In Table 1, we show for different mSUGRA points the cross section of the sum of 
all superpartner pair productions, namely the $R_{p}$ conserving SUSY background of the 3 lepton signature generated by the single chargino production. As can be seen in this table, the summed superpartner pair production rate decreases as $m_{0}$ and $m_{1 / 2}$ increase. This is due to the increase of the superpartner masses as the $m_{0}$ or $m_{1 / 2}$ parameter increases. The SUSY background will be important only for low values of $m_{0}$ and $m_{1 / 2}$ as we will see in the following.

\subsection{Cuts}

In order to simulate the single chargino production $p \bar{p} \rightarrow \tilde{\chi}_{1}^{ \pm} l^{\mp}$ at Tevatron, the matrix elements (see Appendix A) of this process have been implemented in a version of the SUSYGEN event generator [45] allowing the generation of $p \bar{p}$ reactions [46]. The Standard Model background ( $W^{ \pm} Z^{0}, Z^{0} Z^{0}$ and $t \bar{t}$ productions) has been simulated using the PYTHIA event generator [32] and the SUSY background (all SUSY particles pair productions) using the HERWIG event generator [44]. SUSYGEN, PYTHIA and HERWIG have been interfaced with the SHW detector simulation package [37], which mimics an average of the CDF and D0 Run II detector performance.

We have developped a series of cuts in order to enhance the signal-to-background ratio. First, we have selected the events with at least three leptons where the leptons are either an electron, a muon or a tau reconstructed from a jet, namely $N_{l} \geq 3[l=e, \mu, \tau]$. We have also considered the case where the selected leptons are only electrons and muons, namely $N_{l} \geq 3[l=e, \mu]$.

The selection criteria on the jets was to have a number of jets greater or equal to two, where the jets have a transverse momentum higher than $10 \mathrm{GeV}$, namely $N_{j} \geq 2$ with $P_{t}(j)>10 \mathrm{GeV}$. This jet veto reduces the 3 lepton backgrounds coming from the $W^{ \pm} Z^{0}$ and $Z^{0} Z^{0}$ productions. Indeed, the $W^{ \pm} Z^{0}$ production generates no hard jets and the $Z^{0} Z^{0}$ production generates at most one hard jet. Moreover, the hard jet produced in the $Z^{0} Z^{0}$ background is generated by a tau decay (see Section 4.2) and can thus be identified as a tau.

Besides, some effective cuts concerning the energies of the produced leptons have been applied. In Fig.7, we show the distributions of the third leading lepton energy in the 3 lepton events produced by the Standard Model background $\left(W^{ \pm} Z^{0}, Z^{0} Z^{0}\right.$ and $t \bar{t}$ ) and the SUSY signal. Based on those kinds of distributions, we have chosen the following cut on the third leading lepton energy: $E\left(l_{3}\right)>10 \mathrm{GeV}$. Similarly, we have required that the energies of the 2 leading leptons verify $E\left(l_{2}\right)>20 \mathrm{GeV}$ and $E\left(l_{1}\right)>20 \mathrm{GeV}$.

We will refer to all the selection criteria described above, namely $N_{l} \geq 3[l=e, \mu, \tau]$ with $E\left(l_{1}\right)>20 G e V, E\left(l_{2}\right)>20 G e V, E\left(l_{3}\right)>10 G e V$, and $N_{j} \geq 2$ with $P_{t}(j)>10 G e V$, as cut 1 .

Finally, since the leptons originating from the hadron decays (as in the $t \bar{t}$ production) are not well isolated, we have applied some cuts on the lepton isolation. We have imposed the isolation cut $\Delta R=\sqrt{\delta \phi^{2}+\delta \theta^{2}}>0.4$ where $\phi$ is the azimuthal angle and $\theta$ the polar angle between the 3 most energetic charged leptons and the 2 hardest jets. Such a cut is for instance motivated by the distributions shown in Fig.8 of the $\Delta R$ angular difference between the third leading lepton and the second leading jet, in the 3 lepton events generated by the SUSY signal and Standard Model background. We call cut $\Delta R>0.4$ together with cut 1 , cut 2 .

In order to eliminate poorly isolated leptons, we have also required that $E<2 G e V$, where $E$ represents the summed energies of the jets being close to a muon or an electron, 

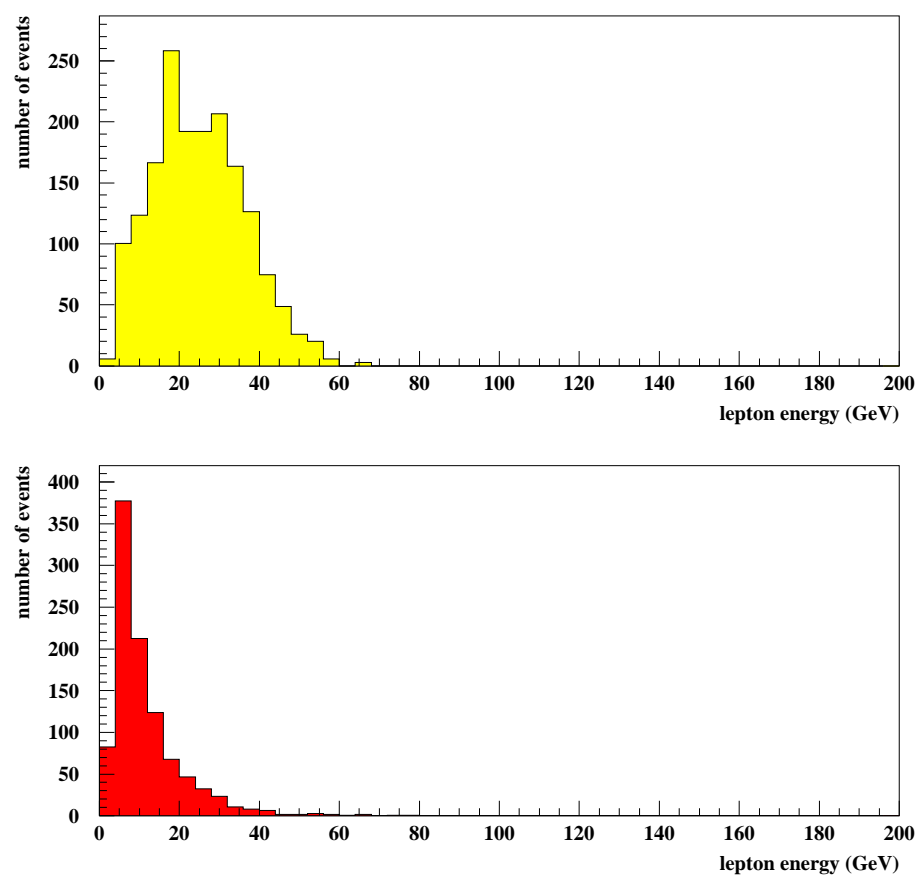

Figure 7: Distributions of the lowest lepton energy (in GeV) among the energies of the 3 leading leptons (electrons and muons) in the events containing at least 3 charged leptons and 2 jets generated by the Standard Model background (lower curve), namely the $W^{ \pm} Z^{0}, Z^{0} Z^{0}$ and $t \bar{t}$ productions, and the SUSY signal (upper curve), for $\lambda_{211}^{\prime}=0.09, M_{2}=150 \mathrm{GeV}$, $m_{0}=200 \mathrm{GeV}, \tan \beta=1.5$ and $\operatorname{sign}(\mu)<0$. The numbers of events correspond to an integrated luminosity of $\mathcal{L}=10 \mathrm{fb}^{-1}$.

namely the jets contained in the cone centered on a muon or an electron and defined by $\Delta R<0.25$. This cut is not applied for taus candidates as they have hadronic decays. It is quite efficient (see Fig.21 for the 2 lepton case) since we sum over all jet energies in the cone. The Standard Model background shows more jets and less separation between jets and leptons in $(\theta, \phi)$ in final state than the single productions ${ }^{1}$. We denote cut $E<2 G e V$ plus cut 2 as cut $3^{2}$.

The selected events require high energy charged leptons and jets and can thus easily be triggered at Tevatron. This is illustrated in Fig.9 where we show the energy distributions of the 3 leptons and the second leading jet in the 3 leptons events selected by applying cut 3 and generated by the SUSY signal and Standard Model background.

In Table 2, we give the numbers of three lepton events expected from the Standard Model background at Tevatron Run II with the various cuts described above. We see in Table 2 that the main source of Standard Model background to the three lepton signature at Tevatron is the $t \bar{t}$ production. This is due to the important cross section of the $t \bar{t}$ production compared to the other Standard Model backgrounds (see Section 4.2). Table 2 also shows that the $t \bar{t}$ background is relatively more suppressed than the other sources of Standard Model background by the lepton isolation cuts. The reason is that in the $t \bar{t}$

\footnotetext{
${ }^{1}$ This cut will have to be fine tuned with real events since it will depend on the energy flow inside the detector, the overlap and minimum biased events.

${ }^{2}$ Although it has not been applied, we mention another kind of isolation cut which allows to further reduce the Standard Model background: $\delta \phi>70^{\circ}$ between the leading charged lepton and the 2 hardest jets.
} 


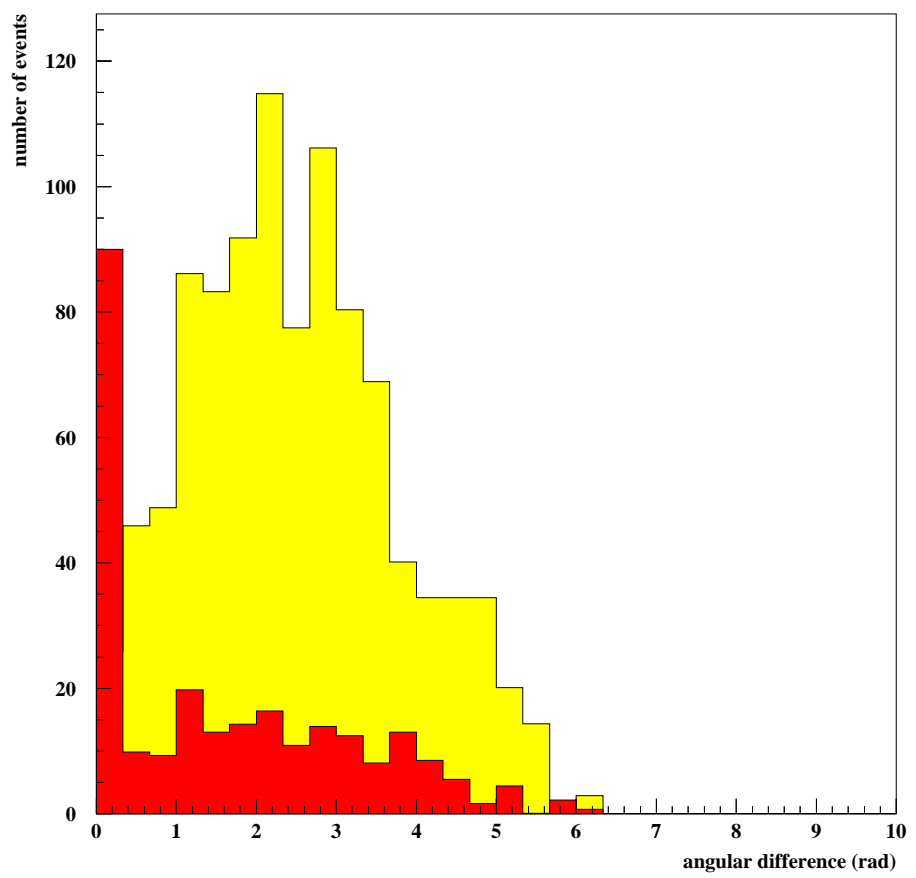

Figure 8: Distributions of the $\Delta R$ angular difference (in rad) between the third leading lepton (electron or muon) and the second leading jet in the 3 leptons events selected by applying cut 1 and produced by the Standard Model background (curve in black), namely the $W^{ \pm} Z^{0}, Z^{0} Z^{0}$ and $t \bar{t}$ productions, and the $S U S Y$ signal (curve in grey), for $\lambda_{211}^{\prime}=0.09$, $M_{2}=150 \mathrm{GeV}, m_{0}=200 \mathrm{GeV}, \tan \beta=1.5$ and $\operatorname{sign}(\mu)<0$. The numbers of events correspond to an integrated luminosity of $\mathcal{L}=10 \mathrm{fb}^{-1}$.

background, one of the 3 charged leptons of the final state is generated in a $b$-jet and is thus not well isolated.

In Table 3, we give the number of three lepton events generated by the SUSY background (all superpartners pair productions) at Tevatron Run II as a function of the $m_{0}$ and $m_{1 / 2}$ parameters for the cut 3 . This number of events decreases as $m_{0}$ and $m_{1 / 2}$ increase due to the behaviour of the summed superpartners pair productions cross section in the SUSY parameter space (see Section 4.3).

\subsection{Results}

\subsubsection{Discovery potential for the $\lambda_{2 j k}^{\prime}$ coupling constant}

We first present the reach in the mSUGRA parameter space obtained from the analysis of the trilepton signature at Tevatron Run II generated by the single chargino production through the $\lambda_{211}^{\prime}$ coupling, namely $p \bar{p} \rightarrow \tilde{\chi}_{1}^{ \pm} \mu^{\mp}$. The sensitivity that can be obtained on the $\lambda_{2 j k}^{\prime}$ ( $j$ and $k$ being not equal to 1 simultaneously) couplings based on the $\tilde{\chi}_{1}^{ \pm} \mu^{\mp}$ production analysis will be discussed at the end of this section for a given mSUGRA point. We give more detailed results for the case of a single dominant $\lambda_{211}^{\prime}$ coupling since this $R_{p}$ coupling gives the highest partonic luminosity to the $\tilde{\chi}_{1}^{ \pm} \mu^{\mp}$ production (see Section 3.1.1) and leads thus to the highest sensitivities. 

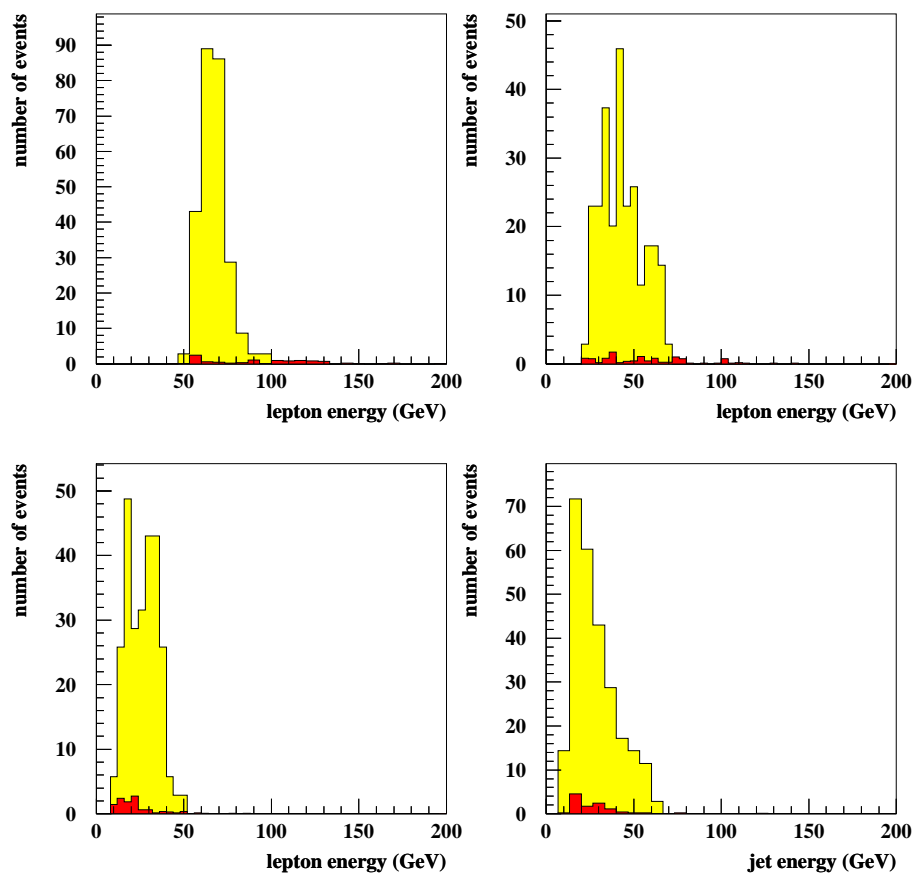

Figure 9: Energy distributions (in GeV) of the 3 leading charged leptons and the second leading jet in the events containing at least 3 charged leptons selected by applying cut 3 and produced by the Standard Model background (curve in black), namely the $W^{ \pm} Z^{0}, Z^{0} Z^{0}$ and $t \bar{t}$ productions, and the SUSY signal (curve in grey), for $\lambda_{211}^{\prime}=0.09, M_{2}=150 G e V$, $m_{0}=200 \mathrm{GeV}, \tan \beta=1.5$ and $\operatorname{sign}(\mu)<0$. The upper left plot represents the leading lepton distribution, the upper right plot the second leading lepton distribution and the lower left plot the third leading lepton distribution. The numbers of events correspond to an integrated luminosity of $\mathcal{L}=10 \mathrm{fb}^{-1}$.

In Fig.10, we present the $3 \sigma$ and $5 \sigma$ discovery contours and the limits at $95 \%$ confidence level in the plane $m_{0}$ versus $m_{1 / 2}$, for $\operatorname{sign}(\mu)<0, \tan \beta=1.5$ and using a set of values for $\lambda_{211}^{\prime}$ and the luminosity. This discovery potential was obtained by considering the $\tilde{\chi}_{1}^{ \pm} \mu^{\mp}$ production and the background originating from the Standard Model. The signal and background were selected by using cut 3 described in Section 4.4. The results presented for a luminosity of $\mathcal{L}=0.5 \mathrm{fb}^{-1}$ in Fig. 10 and Fig. 11 were obtained with cut 2 only in order to optimize the sensitivity on the SUSY parameters. The reduction of the sensitivity on $\lambda_{211}^{\prime}$ observed in Fig.10 when either $m_{0}$ or $m_{1 / 2}$ increases is due to the decrease of the $\tilde{\chi}_{1}^{ \pm} \mu^{\mp}$ production cross section with $m_{0}$ or $m_{1 / 2}$ (or equivalently $M_{2}$ ), which can be observed in Fig.4. In Fig.10, we also see that for all the considered values of $\lambda_{211}^{\prime}$ and the luminosity, the sensitivity on $m_{1 / 2}$ is reduced to low masses in the domain $m_{0} \lesssim 200 \mathrm{GeV}$. This important reduction of the sensitivity as $m_{0}$ decreases is due to the decrease of the phase space factor associated to the decay $\tilde{\nu}_{\mu} \rightarrow \tilde{\chi}^{ \pm} \mu^{\mp}$ (see Section 3.1.1). Finally, we note from Fig. 3 that for $\operatorname{sign}(\mu)>0$ the $\tilde{\chi}_{1}^{ \pm} \mu^{\mp}$ production cross section, and thus the sensitivities presented in Fig.10, would incur a little increase compared to the case $\operatorname{sign}(\mu)<0$.

In Fig.11, the discovery potential is shown in the $\lambda_{211}^{\prime}-m_{0}$ plane for different values of $M_{2}$ and the luminosity. For a given value of $M_{2}$, we note that the sensitivity on the $\lambda_{211}^{\prime}$ coupling decreases at high and low values of $m_{0}$. The main explanation is the decrease 


\begin{tabular}{|c|c|c|c|c|}
\hline & $W^{ \pm} Z^{0}$ & $Z^{0} Z^{0}$ & $t \bar{t}$ & Total \\
\hline cut 1 & $1.39 \pm 0.11$ & $1.37 \pm 0.11$ & $39.80 \pm 1.00$ & $42.56 \pm 1.01$ \\
\hline cut 2 & $0.26 \pm 0.05$ & $0.21 \pm 0.04$ & $4.23 \pm 0.39$ & $4.70 \pm 0.40$ \\
\hline cut 3 & $0.24 \pm 0.04$ & $0.17 \pm 0.04$ & $1.14 \pm 0.17$ & $1.55 \pm 0.18$ \\
\hline cut $1^{\star}$ & $0.51 \pm 0.06$ & $0.73 \pm 0.08$ & $27.80 \pm 0.80$ & $29.04 \pm 0.80$ \\
\hline cut 2 & $0.26 \pm 0.05$ & $0.21 \pm 0.04$ & $2.92 \pm 0.27$ & $3.39 \pm 0.28$ \\
\hline cut $3^{\star}$ & $0.23 \pm 0.04$ & $0.17 \pm 0.04$ & $0.64 \pm 0.13$ & $1.04 \pm 0.14$ \\
\hline
\end{tabular}

Table 2: Numbers of three lepton events generated by the Standard Model background $\left(W^{ \pm} Z^{0}, Z^{0} Z^{0}\right.$ and $t \bar{t}$ productions) at Tevatron Run II for the cuts described in the text, assuming an integrated luminosity of $\mathcal{L}=1 \mathrm{fb}^{-1}$ and a center of mass energy of $\sqrt{s}=2 \mathrm{TeV}$. The cuts marked by a $\star$ do not include the reconstruction of the tau-jets. These results have been obtained by generating and simulating $310^{5}$ events for the $W^{ \pm} Z^{0}$ production, $10^{4}$ events for the $Z^{0} Z^{0}$ and $310^{5}$ events for the $t \bar{t}$.

\begin{tabular}{|c|c|c|c|c|c|}
\hline$m_{1 / 2} \backslash m_{0}$ & $100 \mathrm{GeV}$ & $200 \mathrm{GeV}$ & $300 \mathrm{GeV}$ & $400 \mathrm{GeV}$ & $500 \mathrm{GeV}$ \\
\hline $100 \mathrm{GeV}$ & 93.94 & 125.59 & 80.53 & 66.62 & 63.90 \\
\hline $200 \mathrm{GeV}$ & 5.11 & 4.14 & 3.86 & 4.02 & 4.26 \\
\hline $300 \mathrm{GeV}$ & 2.26 & 0.66 & 0.52 & 0.55 & 0.55 \\
\hline
\end{tabular}

Table 3: Number of 3 lepton events generated by the SUSY background (all superpartners pair productions) at Tevatron Run II as a function of the $m_{0}$ and $m_{1 / 2}$ parameters for $\tan \beta=1.5, \operatorname{sign}(\mu)<0$ and $\lambda_{211}^{\prime}=0.05$. Cut 3 (see text) has been applied. These results have been obtained by generating 7500 events and correspond to an integrated luminosity of $\mathcal{L}=1 \mathrm{fb}^{-1}$ and a center of mass energy of $\sqrt{s}=2 \mathrm{TeV}$.

of the $p \bar{p} \rightarrow \tilde{\chi}_{1}^{ \pm} \mu^{\mp}$ rate at high and low values of $m_{0}$ which appears clearly in Fig.5. We also observe, as in Fig.10, a decrease of the sensitivity on the $\lambda_{211}^{\prime}$ coupling when $M_{2}$ (or equivalently $m_{1 / 2}$ ) increases for a fixed value of $m_{0}$.

The strongest bounds on the supersymmetric masses obtained at LEP in an $R_{p}$ model with a non-vanishing $\lambda^{\prime}$ Yukawa coupling are $m_{\tilde{\chi}_{1}^{0}}>26 G e V$ (for $m_{0}=200 G e V$ and $\tan \beta=\sqrt{2}[47]), m_{\tilde{\chi}_{1}^{ \pm}}>100 \mathrm{GeV}, m_{\tilde{l}}>93 \mathrm{GeV}, m_{\tilde{\nu}}>86 \mathrm{GeV}$ [29]. For the minimum values of $m_{0}$ and $m_{1 / 2}$ spanned by the parameter space described in Figures 10 and 11 , namely $m_{0}=100 \mathrm{GeV}$ and $M_{2}=100 \mathrm{GeV}$, the mass spectrum is $m_{\tilde{\chi}_{1}^{ \pm}}=113 \mathrm{GeV}$, $m_{\tilde{\chi}_{1}^{0}}=54 \mathrm{GeV}, m_{\tilde{\nu}_{L}}=127 \mathrm{GeV}, m_{\tilde{l}_{L}}=137 \mathrm{GeV}, m_{\tilde{l}_{R}}=114 \mathrm{GeV}$, so that we are well above these limits. Since both the scalar and gaugino masses increase with $m_{0}$ and $m_{1 / 2}$, the parameter space described in Figures 10 and 11 lies outside the SUSY parameters ranges excluded by LEP data [29, 47]. Therefore, the discovery potential of Figures 10 and 11 represents an important improvement with respect to the supersymmetric masses limits derived from LEP data [29, 47]. Figures 10 and 11 show also that the low-energy bound on the considered $R_{p}$ coupling, $\lambda_{211}^{\prime}<0.09\left(m_{\tilde{d}_{R}} / 100 G e V\right)$ at $1 \sigma$ (from $\pi$ decay) [4], can be greatly improved.

Interesting sensitivities on the SUSY parameters can already be obtained within the first year of Run II at Tevatron with a low luminosity $\left(\mathcal{L}=0.5 \mathrm{fb}^{-1}\right)$ and no reconstruction of the tau-jets. To illustrate this point, we present in Fig. 12 and Fig.13 the same discovery potentials as in Fig.10 and Fig.11, respectively, obtained without reconstruction of the tau leptons decaying into jets. By comparing Fig.10, Fig.11 and Fig.12, Fig.13, we observe 


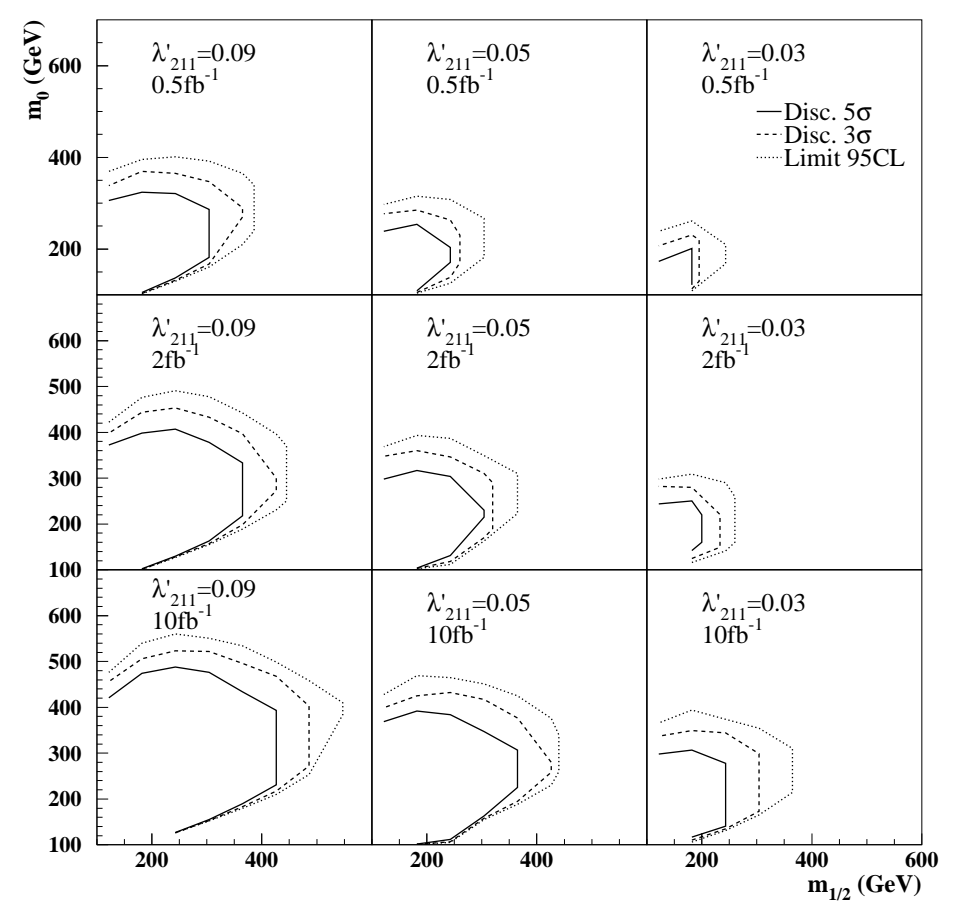

Figure 10: Discovery contours at $5 \sigma$ (full line), $3 \sigma$ (dashed line) and limit at 95\% C.L. (dotted line) obtained from the trilepton signature analysis at Tevatron Run II assuming a center of mass energy of $\sqrt{s}=2 \mathrm{TeV}$. This discovery reach is presented in the plane $m_{0}$ versus $m_{1 / 2}$, for $\operatorname{sign}(\mu)<0, \tan \beta=1.5$ and different values of $\lambda_{211}^{\prime}$ and of luminosity.

that the sensitivity on the SUSY parameters is weakly affected by the reconstruction of the tau-jets ${ }^{3}$.

Using the ratios of the cross sections for the $\tilde{\chi}_{1}^{+} \mu^{-}$productions via different $\lambda_{2 j k}^{\prime}$ couplings, one can deduce from the sensitivity obtained on $\lambda_{211}^{\prime}$ via the 3 lepton final state analysis an estimation of the sensitivity on any $\lambda_{2 j k}^{\prime}$ coupling. For instance, let us consider the SUSY point $m_{0}=180 \mathrm{GeV}, M_{2}=200 \mathrm{GeV}, \tan \beta=1.5$ and $\mu=-200 \mathrm{GeV}$ $\left(m_{\tilde{u}_{L}}=601 \mathrm{GeV}, m_{\tilde{d}_{L}}=603 \mathrm{GeV}, m_{\tilde{u}_{R}}=582 \mathrm{GeV}, m_{\tilde{d}_{R}}=580 \mathrm{GeV}, m_{\tilde{l}_{L}}=253 \mathrm{GeV}\right.$, $\left.m_{\tilde{l}_{R}}=205 \mathrm{GeV} m_{\tilde{\nu}_{L}}=248 \mathrm{GeV}, m_{\tilde{\chi}_{1}^{ \pm}}=199 \mathrm{GeV}, m_{\tilde{\chi}_{1}^{0}}=105 \mathrm{GeV}\right)$ which corresponds, as can be seen in Fig.11, to the point where the sensitivity on $\lambda_{211}^{\prime}$ is maximized for $M_{2}=200 \mathrm{GeV}$. We can see on Fig.5 that for this SUSY point, the ratio between the rates of the $\tilde{\chi}_{1}^{+} \mu^{-}$productions via $\lambda_{211}^{\prime}$ and $\lambda_{221}^{\prime}$ is $\sigma\left(\lambda_{211}^{\prime}\right) / \sigma\left(\lambda_{221}^{\prime}\right) \approx 7.9$ for same values of the $R_{p}$ couplings. Therefore, since the single chargino production rate scales as $\lambda^{\prime 2}$ (see Appendix A), the sensitivity on $\lambda_{221}^{\prime}$ at this SUSY point is equal to the sensitivity obtained on $\lambda_{211}^{\prime}\left(\sim 0.02\right.$ at $95 \% C L$ with $\mathcal{L}=2 f b^{-1}$ as shows Fig.11) multiplied by the factor $\sqrt{7.9}$, namely $\sim 0.05$. This result represents a significant improvement with respect to

\footnotetext{
${ }^{3}$ This is actually an artefact of the method: cut 3 is our most efficient cut to reduce the Standard Model background with electrons and muons but is not applied with taus. Thus, the relative ratio signal over background is not so good with taus. Finding another efficient cut could improve our discovery potential and limits using taus.
} 


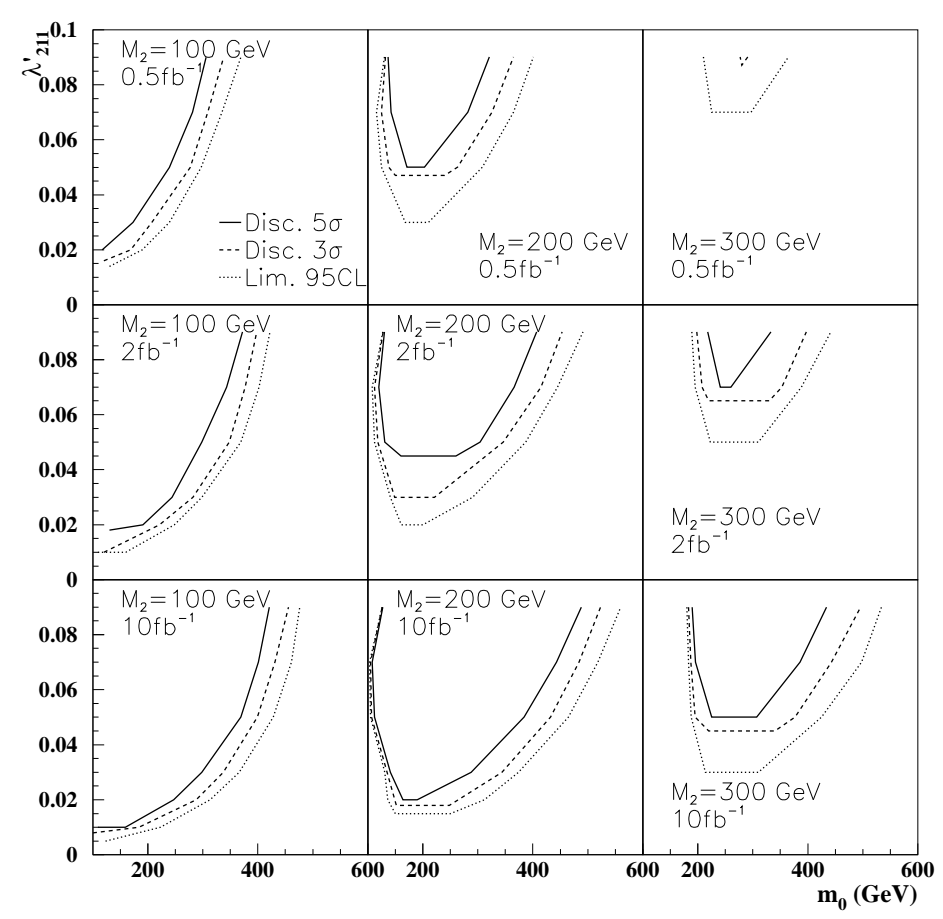

Figure 11: Discovery contours at $5 \sigma$ (full line), $3 \sigma$ (dashed line) and limit at 95\% C.L. (dotted line) presented in the plane $\lambda_{211}^{\prime}$ versus the $m_{0}$ parameter, for $\operatorname{sign}(\mu)<0, \tan \beta=1.5$ and different values of $M_{2}$ and of luminosity.

the low-energy indirect limit $\lambda_{221}^{\prime}<0.18\left(m_{\tilde{d}_{R}} / 100 \mathrm{GeV}\right)$ [4]. Using the same method, we find at the same SUSY point the sensitivities on the $\lambda_{2 j k}^{\prime}$ coupling constants given in Table 4. All the sensitivities on the $\lambda_{2 j k}^{\prime}$ coupling constants given in Table 4 are stronger than the low-energy bounds of [4] which we rewrite here: $\lambda_{21 k}^{\prime}<0.09\left(m_{\tilde{d}_{k R}} / 100 \mathrm{GeV}\right)$ at $1 \sigma(\pi$ decay), $\lambda_{22 k}^{\prime}<0.18\left(m_{\tilde{d}_{k R}} / 100 \mathrm{GeV}\right)$ at $1 \sigma(D$ decay $), \lambda_{231}^{\prime}<0.22\left(m_{\tilde{b}_{L}} / 100 \mathrm{GeV}\right)$ at $2 \sigma\left(\nu_{\mu}\right.$ deep inelastic scattering $), \lambda_{232}^{\prime}<0.36\left(m_{\tilde{q}} / 100 \mathrm{GeV}\right)$ at $1 \sigma\left(R_{\mu}\right), \lambda_{233}^{\prime}<0.36\left(m_{\tilde{q}} / 100 \mathrm{GeV}\right)$ at $1 \sigma\left(R_{\mu}\right)$.

In the case of a single dominant $\lambda_{2 j 3}^{\prime}$ coupling, the neutralino decays as $\tilde{\chi}_{1}^{0} \rightarrow \mu u_{j} b$ and the semileptonic decay of the b-quark could affect the analysis efficiency. Therefore in this case, the precise sensitivity cannot be simply calculated by scaling the value obtained for $\lambda_{211}^{\prime}$. Nevertheless, the order of magnitude of the sensitivity which can be inferred from our analysis should be correct.

The range of SUSY parameters in which the constraint on a given $\lambda_{2 j k}^{\prime}$ coupling constant obtained via the three leptons analysis is stronger than the relevant low-energy bound depends on the low-energy bound itself as well as on the values of the cross section for the single chargino production via the considered $\lambda_{2 j k}^{\prime}$ coupling.

Finally, we remark that while the low-energy constraints on the $\lambda_{2 j k}^{\prime}$ couplings become weaker as the squark masses increase, the sensitivities on those couplings obtained from 


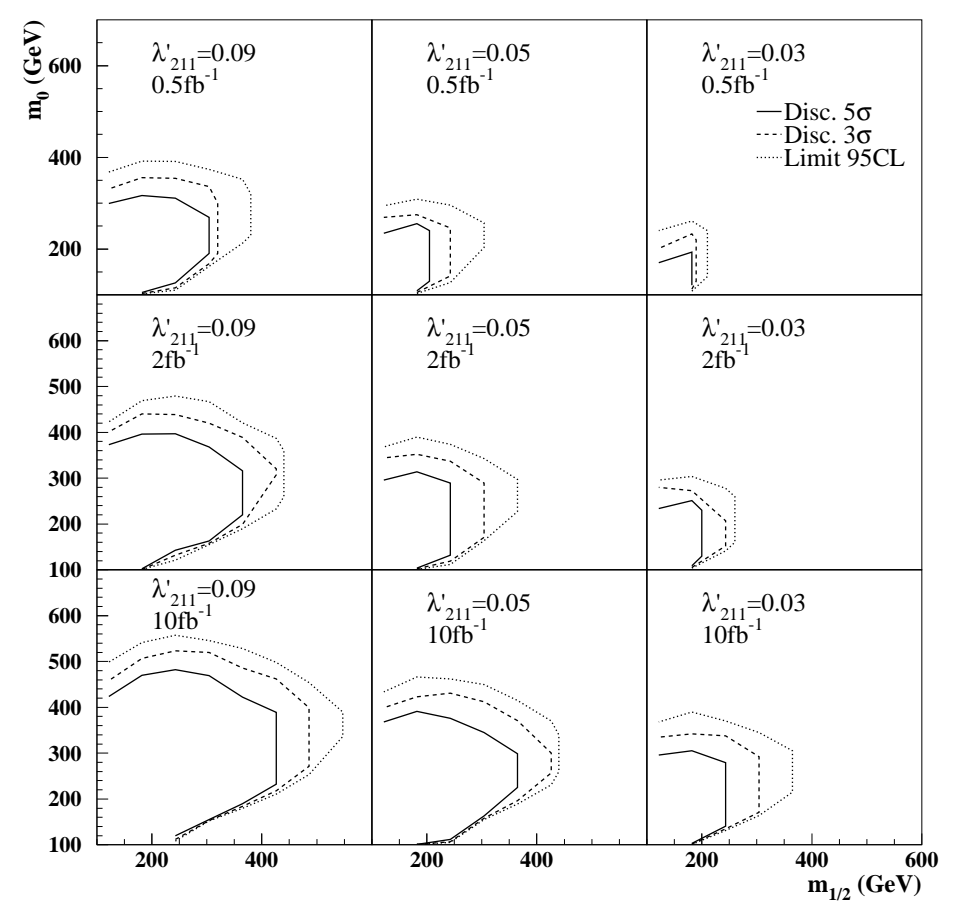

Figure 12: Discovery contours at $5 \sigma$ (full line), $3 \sigma$ (dashed line) and limit at 95\% C.L. (dotted line) presented in the plane $m_{0}$ versus $m_{1 / 2}$ and obtained without reconstruction of the tau leptons decaying into jets for $\operatorname{sign}(\mu)<0, \tan \beta=1.5$ and different values of $\lambda_{211}^{\prime}$ and of luminosity.

the three leptons analysis are essentially independent of the squark masses as long as $m_{\tilde{q}}>m_{\tilde{\chi}_{1}^{ \pm}}$(recall that the branching ratio of the decay $\tilde{\chi}_{1}^{ \pm} \rightarrow q \bar{q} \tilde{\chi}_{1}^{0}$ is greatly enhanced when $\left.m_{\tilde{q}}<m_{\tilde{\chi}_{1}^{ \pm}}\right)$.

We end this section by some comments on the effect of the supersymmetric $R_{p}$ conserving background to the 3 lepton signature. In order to illustrate this discussion, we consider the results on the $\lambda_{211}^{\prime}$ coupling constant.

We see from Table 3 that the SUSY background to the 3 lepton final state can affect the sensitivity on the $\lambda_{211}^{\prime}$ coupling constant obtained by considering only the Standard Model background, which is shown in Fig.10, only in the region of small superpartner masses, namely in the domain $m_{1 / 2} \lesssim 300 \mathrm{GeV}$ for $\tan \beta=1.5, \operatorname{sign}(\mu)<0$ and assuming a luminosity of $\mathcal{L}=1 \mathrm{fb}^{-1}$.

In contrast with the SUSY signal amplitude which is increased if $\lambda_{211}^{\prime}$ is enhanced, the SUSY background amplitude is typically independent on the value of the $\lambda_{211}^{\prime}$ coupling constant since the superpartner pair production does not involve $R_{p}$ couplings. Therefore, even if we consider the SUSY background in addition to the Standard Model one, it is still true that large values of the $\lambda_{211}^{\prime}$ coupling can be probed over a wider domain of the SUSY parameter space than low values, as can be observed in Fig.10 for $m_{1 / 2} \gtrsim 300 \mathrm{GeV}$. Note that in Fig.10 larger values of $\lambda_{211}^{\prime}$ could have been considered as the low-energy 


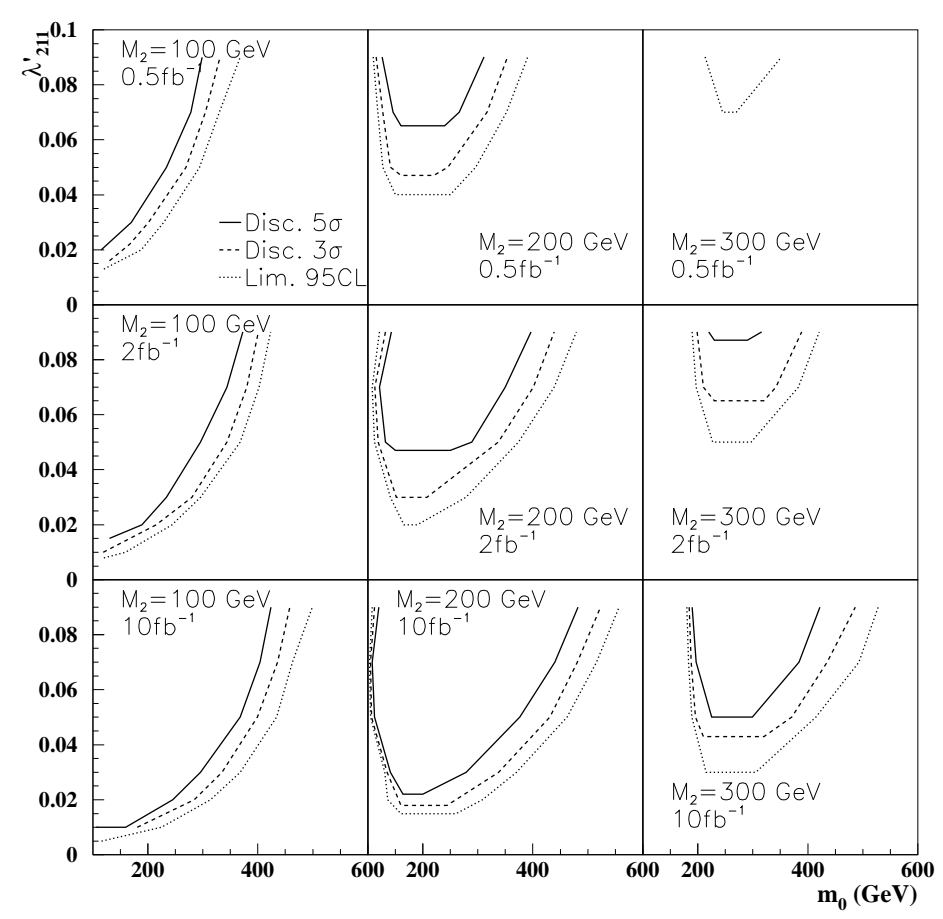

Figure 13: Discovery contours at $5 \sigma$ (full line), $3 \sigma$ (dashed line) and limit at 95\% C.L. (dotted line) presented in the plane $\lambda_{211}^{\prime}$ versus the $m_{0}$ parameter and obtained without reconstruction of the tau leptons decaying into jets for $\operatorname{sign}(\mu)<0, \tan \beta=1.5$ and different values of $M_{2}$ and of luminosity.

bound on this $R_{p}$ coupling, namely $\lambda_{211}^{\prime}<0.09\left(m_{\tilde{d}_{R}} / 100 \mathrm{GeV}\right)$ [4], is proportional to the squark mass.

Finally, we mention that further cuts, as for instance some cuts based on the superpartner mass reconstructions (see Section 4.5.4), could allow to reduce the SUSY background to the 3 lepton signature.

\subsubsection{High $\tan \beta$ scenario}

In mSUGRA, for large values of $\tan \beta$ and small values of $m_{0}$ compared to $m_{1 / 2}$, due to the large mixing in the third generation sfermions, the mass of the lighter $\tilde{\tau}_{1}$ slepton can become smaller than $m_{\tilde{\chi}_{1}^{ \pm}}$, with the sneutrino remaining heavier than the $\tilde{\chi}_{1}^{ \pm}$so that the $\tilde{\chi}_{1}^{ \pm} l^{\mp}$ production rate can still be significant. In this situation, the efficiency for the 3 lepton signature arising mainly through, $\tilde{\chi}_{1}^{ \pm} \rightarrow \tilde{\tau}_{1}^{ \pm} \nu_{\tau}, \tilde{\tau}_{1}^{ \pm} \rightarrow \tilde{\chi}_{1}^{0} \tau^{ \pm}, \tilde{\chi}_{1}^{0} \rightarrow l_{i}^{ \pm} u_{j} d_{k}$, can be enhanced compared to the case where the 3 lepton signal comes from, $\tilde{\chi}_{1}^{ \pm} \rightarrow$ $\tilde{\chi}_{1}^{0} l^{ \pm} \nu, \tilde{\chi}_{1}^{0} \rightarrow l_{i}^{ \pm} u_{j} d_{k}$. Indeed, the branching ratio $B\left(\tilde{\chi}_{1}^{ \pm} \rightarrow \tilde{\tau}_{1}^{ \pm} \nu_{\tau}\right)$ can reach $\sim 100 \%$, $B\left(\tilde{\tau}_{1}^{ \pm} \rightarrow \tilde{\chi}_{1}^{0} \tau^{ \pm}\right) \approx 100 \%$, since the $\tilde{\chi}_{1}^{0}$ is the LSP, $B\left(\tau \rightarrow l \nu_{l} \nu_{\tau}\right)=35 \%(l=e, \mu)$ and the $\tau$-jets can be reconstructed at Tevatron Run II. However, in such a scenario the increased number of tau leptons in the final state leads to a softer charged lepton spectrum which tends to reduce the efficiency after cuts. Therefore, for relatively small values of 


\begin{tabular}{|l|l|l|l|l|l|l|l|}
\hline$\lambda_{212}^{\prime}$ & $\lambda_{213}^{\prime}$ & $\lambda_{221}^{\prime}$ & $\lambda_{222}^{\prime}$ & $\lambda_{223}^{\prime}$ & $\lambda_{231}^{\prime}$ & $\lambda_{232}^{\prime}$ & $\lambda_{233}^{\prime}$ \\
\hline 0.04 & 0.07 & 0.05 & 0.12 & 0.21 & 0.10 & 0.36 & 0.63 \\
\hline
\end{tabular}

Table 4: Sensitivities at $95 \% C L$ on the $\lambda_{2 j k}^{\prime}$ coupling constants derived from the sensitivity on $\lambda_{211}^{\prime}$ for a luminosity of $\mathcal{L}=2 \mathrm{fb}^{-1}$ and the following set of SUSY parameters, $\tan \beta=1.5, M_{2}=200 \mathrm{GeV}$, $\mu=-200 \mathrm{GeV}$ and $m_{0}=180 \mathrm{GeV}$.

$m_{0}$ compared to $m_{1 / 2}$, the sensitivity obtained in the high $\tan \beta$ scenario is essentially unaffected with respect to the low $\tan \beta$ situation, unless $m_{0}$ is small enough to render $m_{\tilde{\tau}_{1}}$ and $m_{\tilde{\chi}_{1}^{0}}$ almost degenerate. As a matter of fact, in such a situation, the energy of the tau produced in the decay $\tilde{\tau}_{1}^{ \pm} \rightarrow \tilde{\chi}_{1}^{0} \tau^{ \pm}$often falls below the analysis cuts. Therefore, this degeneracy results in a loss of signal efficiency after cuts, at small values of $m_{0}$ compared to $m_{1 / 2}$, and thus in a loss of sensitivity, with respect to the low $\tan \beta$ situation. This can be seen by comparing Fig.10, Fig.11 and Fig.14, Fig.15. Indeed, the decrease of the sensitivity on $m_{1 / 2}$ at low $m_{0}$ is stronger for high $\tan \beta$ (see Fig.14) than for low $\tan \beta$ (see Fig.10). Similarly, the decrease of the sensitivity on $\lambda_{211}^{\prime}$ at low $m_{0}$ is stronger for high $\tan \beta$ (see Fig.15) than for low $\tan \beta$ (see Fig.11).

The effect on the discovery potential of the single chargino production rate increase at large $\tan \beta$ values shown in Fig. 2 is hidden by the large $\tan \beta$ scenario influences on the cascade decays described above.

In contrast with the low $\tan \beta$ scenario (see Section 4.5.1), the sensitivity on the SUSY parameters depends in a significant way on the reconstruction of the tau-jets in case where $\tan \beta$ is large, as can be seen in Fig.14 and Fig.15. The reason is the increased number of tau leptons among the final state particles in a large $\tan \beta$ model. This is due to the decrease of the lighter stau mass which tends to increase the $B\left(\tilde{\chi}_{1}^{ \pm} \rightarrow \tilde{\chi}_{1}^{0} \tau^{ \pm} \nu_{\tau}\right)$ branching ratio.

\subsubsection{Discovery potential for the $\lambda_{1 j k}^{\prime}$ and $\lambda_{3 j k}^{\prime}$ coupling constants}

In Fig.16, we present the $3 \sigma$ and $5 \sigma$ discovery contours and the limits at $95 \%$ confidence level in the plane $m_{0}$ versus $m_{1 / 2}$, for $\operatorname{sign}(\mu)<0, \tan \beta=1.5, \lambda_{311}^{\prime}=0.10$ and various values of the luminosity. In Fig.17, the discovery potential is shown in the $\lambda_{311}^{\prime}-m_{0}$ plane for $M_{2}=200 \mathrm{GeV}$. Comparing Fig.16, Fig.17 and Fig.10, Fig.11, we see that the sensitivity on the SUSY parameters is weaker in the case of a single dominant $\lambda_{311}^{\prime}$ coupling than in the case of a single dominant $\lambda_{211}^{\prime}$ coupling. The reason is that in the case of a single dominant $\lambda_{3 j k}^{\prime}$ coupling constant, tau leptons are systematically produced at the chargino production level $p \bar{p} \rightarrow \tilde{\chi}_{1}^{ \pm} \tau^{\mp}$ (see Fig.1(a)) as well as in the LSP decay $\tilde{\chi}_{1}^{0} \rightarrow \tau u_{j} d_{k}$ (see Section 4.1), so that the number of tau leptons among the 3 charged leptons of the final state is increased compared to the dominant $\lambda_{2 j k}^{\prime}$ case. The decrease in sensitivity is due to the fact that a lepton (electron or muon) generated in a tau decay has an higher probability not to pass the analysis requirements concerning the particle energy and that the reconstruction efficiency for a tau decaying into a jet is limited.

Nevertheless, the discovery potentials of Fig.16 and Fig.17 represent also an important improvement with respect to the experimental mass limits from LEP measurements $[29,47]$ and to the low-energy indirect constraint $\lambda_{311}^{\prime}<0.10\left(m_{\tilde{d}_{R}} / 100 \mathrm{GeV}\right.$ ) at $1 \sigma$ (from $\left.\tau^{-} \rightarrow \pi^{-} \nu_{\tau}\right)$ [4].

We also observe in Fig.16 and Fig.17 that the results obtained from the $\tilde{\chi}_{1}^{ \pm} \tau^{\mp}$ production 


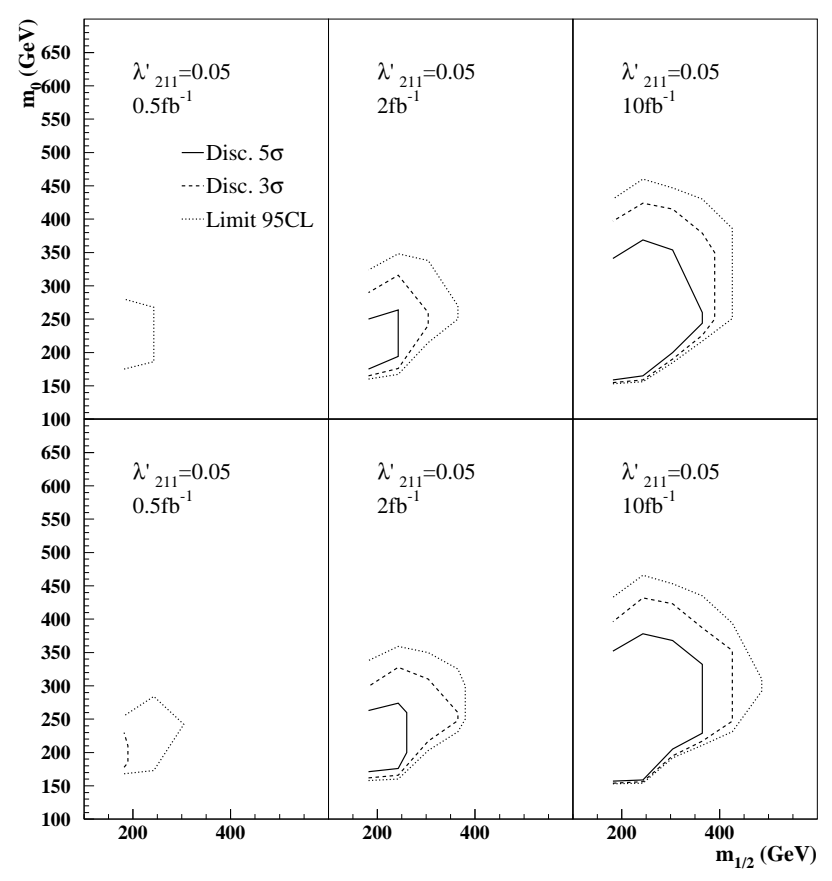

Figure 14: Discovery contours at $5 \sigma$ (full line), $3 \sigma$ (dashed line) and limit at $95 \%$ C.L. (dotted line) presented in the plane $m_{0}$ versus $m_{1 / 2}$, for $\operatorname{sign}(\mu)<0, \tan \beta=50, \lambda_{211}^{\prime}=0.09$ and different values of luminosity. The upper (lower) curves are obtained without (with) the reconstruction of the tau-jets.

analysis in the case of a single dominant $\lambda_{3 j k}^{\prime}$ coupling depend strongly on the reconstruction of the tau-jets. This is due to the large number of tau leptons among the 3 charged leptons of the considered final state.

Using the same method and same SUSY point as in Section 4.5.1, we have estimated the sensitivity on all the $\lambda_{3 j k}^{\prime}$ coupling constants from the sensitivity obtained on $\lambda_{311}^{\prime}$ at $95 \% C L$ for a luminosity of $\mathcal{L}=2 f b^{-1}$. The results are given in Table 5. All the sensitivities on the $R_{p}$ couplings presented in Table 5, except those on $\lambda_{32 k}^{\prime}$, are stronger than the present indirect limits on the same $R_{p}$ couplings: $\lambda_{31 k}^{\prime}<0.10\left(m_{\tilde{d}_{k R}} / 100 \mathrm{GeV}\right)$ at $1 \sigma\left(\tau^{-} \rightarrow \pi^{-} \nu_{\tau}\right), \lambda_{32 k}^{\prime}<0.20\left(\right.$ for $\left.m_{\tilde{l}}=m_{\tilde{q}}=100 \mathrm{GeV}\right)$ at $1 \sigma\left(D^{0}-\bar{D}^{0} \mathrm{mix}\right)$, $\lambda_{33 k}^{\prime}<0.48\left(m_{\tilde{q}} / 100 \mathrm{GeV}\right)$ at $1 \sigma\left(R_{\tau}\right)[4]$.

We mention that in the case of a single dominant $\lambda_{3 j 3}^{\prime}$ coupling, the neutralino decays as $\tilde{\chi}_{1}^{0} \rightarrow \tau u_{j} b$ so that the $\mathrm{b}$ semileptonic decay could affect a little the analysis efficiency.

We discuss now the sensitivities that could be obtained on a single dominant $\lambda_{1 j k}^{\prime}$ coupling constant via the analysis of the reaction $p \bar{p} \rightarrow \tilde{\chi}_{1}^{ \pm} e^{\mp}$ (see Fig.1(a)). Since the cross section of the $\tilde{\chi}_{1}^{ \pm} e^{\mp}$ production through $\lambda_{1 j k}^{\prime}$ is equal to the rate of the $\tilde{\chi}_{1}^{ \pm} \mu^{\mp}$ production via $\lambda_{2 j k}^{\prime}$, for same $j$ and $k$ indices (see Section 3.1.1), the sensitivity obtained on a $\lambda_{1 j k}^{\prime}$ coupling constant is expected to be identical to the sensitivity on $\lambda_{2 j k}^{\prime}$. If we assume that the sensitivities obtained on the $\lambda_{1 j k}^{\prime}$ couplings are equal to those presented in Table 4, we remark that for the SUSY point chosen in this table only the sensitivities expected for the $\lambda_{112}^{\prime}, \lambda_{113}^{\prime}, \lambda_{121}^{\prime}, \lambda_{131}^{\prime}$ and $\lambda_{132}^{\prime}$ couplings are stronger than the corresponding low-energy bounds: $\lambda_{11 k}^{\prime}<0.02\left(m_{\tilde{d}_{k R}} / 100 \mathrm{GeV}\right)$ at $2 \sigma$ (Charged current 


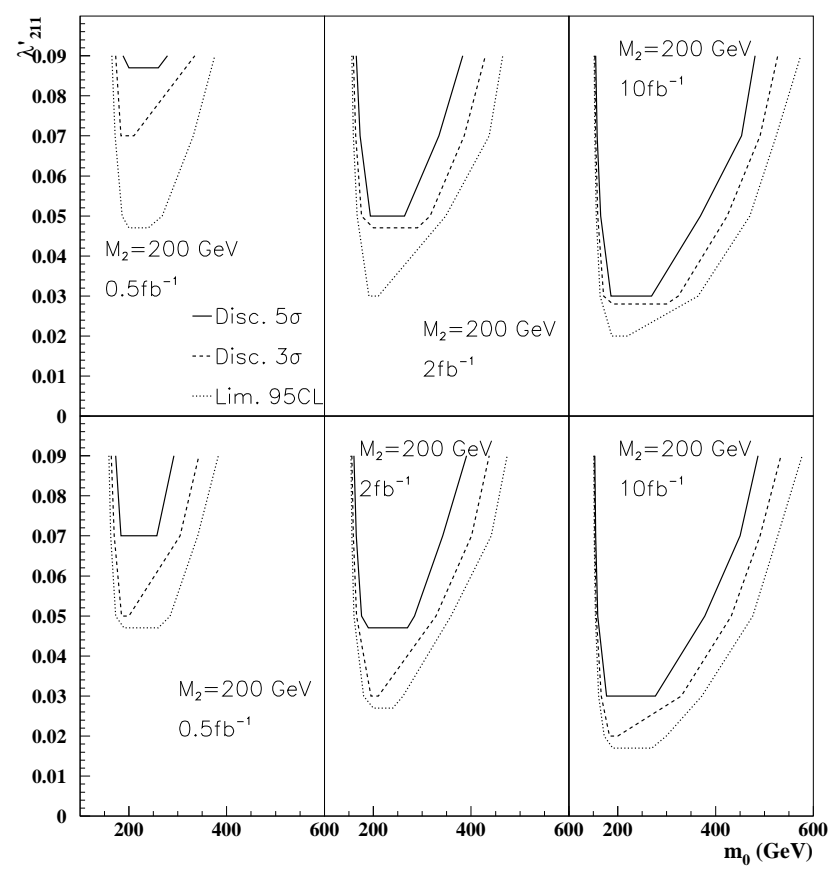

Figure 15: Discovery contours at $5 \sigma$ (full line), $3 \sigma$ (dashed line) and limit at 95\% C.L. (dotted line) presented in the plane $\lambda_{211}^{\prime}$ versus the $m_{0}$ parameter, for $\operatorname{sign}(\mu)<0, \tan \beta=50, M_{2}=200 \mathrm{GeV}$ and different values of luminosity. The upper (lower) curves are obtained without (with) the reconstruction of the tau-jets.

universality), $\lambda_{1 j 1}^{\prime}<0.035\left(m_{\tilde{q}_{j L}} / 100 \mathrm{GeV}\right)$ at $2 \sigma$ (Atomic parity violation), $\lambda_{132}^{\prime}<0.34$ at $1 \sigma$ for $m_{\tilde{q}}=100 \mathrm{GeV}\left(R_{e}\right)$ [4]. The reason is that the low-energy constraints on the $\lambda_{1 j k}^{\prime}$ couplings are typically more stringent than the limits on the $\lambda_{2 j k}^{\prime}$ couplings [4].

\subsubsection{Mass reconstructions}

The $\tilde{\chi}_{1}^{0}$ neutralino decays in our framework as $\tilde{\chi}_{1}^{0} \rightarrow l_{i} u_{j} d_{k}$ through the $\lambda_{i j k}^{\prime}$ coupling constant. The invariant mass distribution of the lepton and the 2 jets coming from this decay channel is peaked at the $\tilde{\chi}_{1}^{0}$ mass. The experimental analysis of this invariant mass distribution would thus be particularly interesting since it would allow a model independent determination of the lightest neutralino mass.

We have performed the $\tilde{\chi}_{1}^{0}$ mass reconstruction based on the 3 lepton signature analysis. The difficulty of this mass reconstruction lies in the selection of the lepton and the 2 jets coming from the $\tilde{\chi}_{1}^{0}$ decay. In the signal we are considering, the only jets come from the $\tilde{\chi}_{1}^{0}$ decay, and of course from the initial and final QCD radiations. Therefore, if there are more than 2 jets in the final state we have selected the 2 hardest ones. It is more subtle for the selection of the lepton since our signal contains 3 leptons. We have considered the case of a single dominant coupling of type $\lambda_{2 j k}^{\prime}$ and focused on the $e \mu \mu$ final state. In these events, one of the $\mu^{ \pm}$is generated in the decay of the produced sneutrino as $\tilde{\nu}_{\mu} \rightarrow \tilde{\chi}_{1}^{ \pm} \mu^{\mp}$ and the other one in the decay of the $\tilde{\chi}_{1}^{0}$ as $\tilde{\chi}_{1}^{0} \rightarrow \mu^{ \pm} u_{j} d_{k}$, while the electron comes from the chargino decay $\tilde{\chi}_{1}^{ \pm} \rightarrow \tilde{\chi}_{1}^{0} e^{ \pm} \nu_{e}$. Indeed, the dominant contribution to the single 


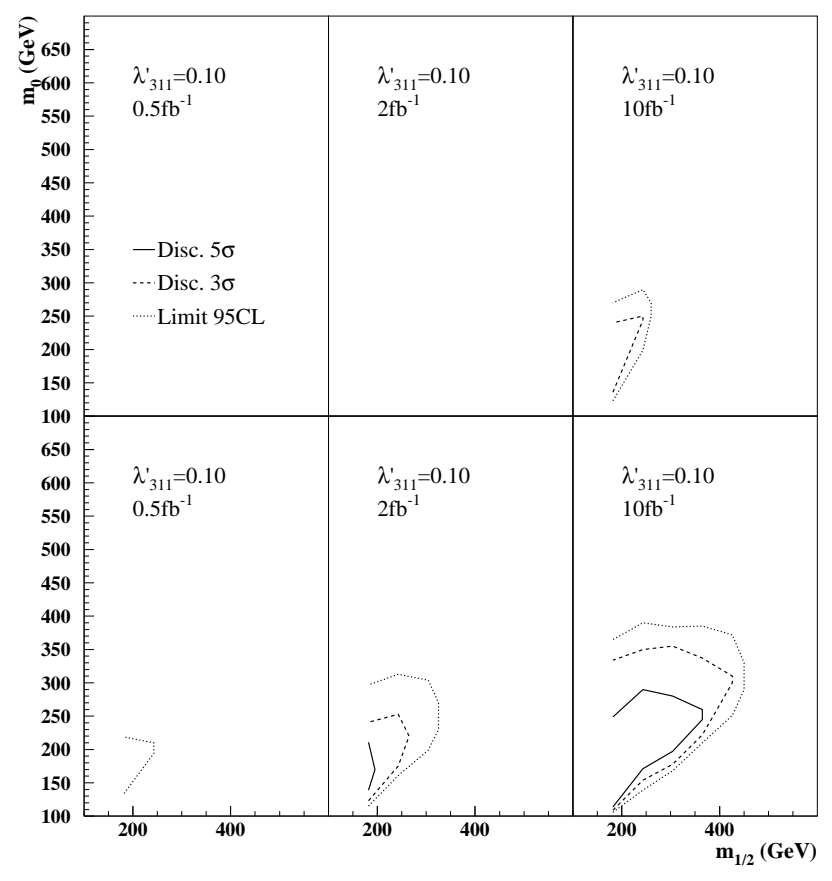

Figure 16: Discovery contours at $5 \sigma$ (full line), $3 \sigma$ (dashed line) and limit at $95 \%$ C.L. (dotted line) presented in the plane $m_{0}$ versus $m_{1 / 2}$, for $\operatorname{sign}(\mu)<0, \tan \beta=1.5, \lambda_{311}^{\prime}=0.10$ and different values of luminosity. The upper (lower) curves are obtained without (with) the reconstruction of the tau-jets.

chargino production is the resonant sneutrino production (see Fig.1). In order to select the muon from the $\tilde{\chi}_{1}^{0}$ decay we have chosen the softer muon, since for relatively important values of the $m_{\tilde{\nu}_{\mu}}-m_{\tilde{\chi}_{1}^{ \pm}}$mass difference the muon generated in the sneutrino decay is the most energetic. Notice that for too degenerate $\tilde{\nu}_{\mu}$ and $\tilde{\chi}_{1}^{ \pm}$masses, the sensitivity on the SUSY parameters suffers a strong decrease as shown in Section 4.5.1.

We present in Fig.18 the invariant mass distribution of the muon and the 2 jets produced in the $\tilde{\chi}_{1}^{0}$ decay. This distribution has been obtained by using the selection criteria described above and by considering the mSUGRA point: $m_{0}=200 \mathrm{GeV}, M_{2}=150 \mathrm{GeV}$, $\tan \beta=1.5, \operatorname{sign}(\mu)<0$ and $\lambda_{211}^{\prime}=0.09\left(m_{\tilde{\chi}_{1}^{0}}=77.7 \mathrm{GeV}, m_{\tilde{\chi}_{1}^{ \pm}}=158.3 \mathrm{GeV}, m_{\tilde{\nu}_{L}}=\right.$ $236 \mathrm{GeV})$. We also show on the plot of Fig.18 the fit of the invariant mass distribution. As can be seen from this fit, the distribution is well peaked around the $\tilde{\chi}_{1}^{0}$ generated mass. The average reconstructed $\tilde{\chi}_{1}^{0}$ mass is of $71 \pm 9 \mathrm{GeV}$.

We have also performed the $\tilde{\chi}_{1}^{ \pm}$and $\tilde{\nu}_{\mu}$ mass reconstructions based on the 3 lepton signature analysis in the scenario of a single dominant coupling of type $\lambda_{2 j k}^{\prime}$. The $\tilde{\chi}_{1}^{ \pm}$and $\tilde{\nu}_{\mu}$ masses reconstructions are based on the 4-momentum of the neutrino present in the $3 l+2 j+\nu$ final state (see Section 4.1). The transverse component of this momentum can be deduced from the momentum of the charged leptons and jets present in the final state. However, the longitudinal component of the neutrino momentum remains unknown due to the poor detection at small polar angle values. Therefore, in this study we have assumed a vanishing longitudinal component of the neutrino momentum. Besides, we have focused on the $e \mu \mu$ events as in the $\tilde{\chi}_{1}^{0}$ mass reconstruction study. In this context, the 


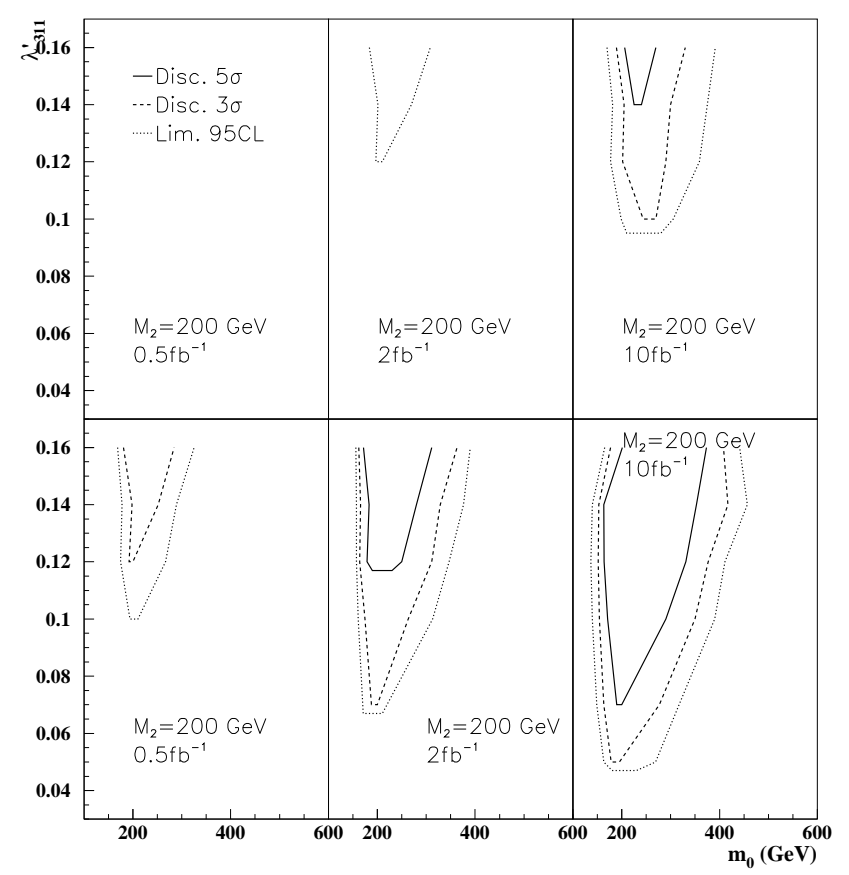

Figure 17: Discovery contours at $5 \sigma$ (full line), $3 \sigma$ (dashed line) and limit at 95\% C.L. (dotted line) presented in the plane $\lambda_{311}^{\prime}$ versus the $m_{0}$ parameter, for $\operatorname{sign}(\mu)<0, \tan \beta=1.5, M_{2}=200 \mathrm{GeV}$ and different values of luminosity. The upper (lower) curves are obtained without (with) the reconstruction of the tau-jets.

cascade decay initiated by the produced lightest chargino is $\tilde{\chi}_{1}^{ \pm} \rightarrow \tilde{\chi}_{1}^{0} e^{ \pm} \nu_{e}, \tilde{\chi}_{1}^{0} \rightarrow \mu^{ \pm} u_{j} d_{k}$. Therefore, the $\tilde{\chi}_{1}^{ \pm}$has been reconstructed from the softer muon, the 2 jets, the electron and the neutrino present in the final state, since the softer muon is mainly generated in the $\tilde{\chi}_{1}^{0}$ decay as explained above. The $\tilde{\nu}_{\mu}$ has then been reconstructed from the $\tilde{\chi}_{1}^{ \pm}$and the leading muon of the final state. This was motivated by the fact that the dominant contribution to the single chargino production is the reaction $p \bar{p} \rightarrow \tilde{\nu}_{\mu} \rightarrow \tilde{\chi}_{1}^{ \pm} \mu^{\mp}$ (see Fig.1).

In Fig.19, we present the $\tilde{\chi}_{1}^{ \pm}$and $\tilde{\nu}_{\mu}$ mass reconstructions performed through the method presented above. We also show on the plots of Fig.19 the fits of the invariant mass distributions. As can be seen from those fits, the distributions are well peaked around the $\tilde{\chi}_{1}^{ \pm}$and $\tilde{\nu}_{\mu L}$ generated masses. The average reconstructed masses are $m_{\tilde{\chi}_{1}^{ \pm}}=171 \pm 35 \mathrm{GeV}$ and $m_{\tilde{\nu}_{\mu L}}=246 \pm 32 \mathrm{GeV}$. This study on the $\tilde{\chi}_{1}^{ \pm}$and $\tilde{\nu}_{\mu L}$ masses shows that based on a simplified mass reconstruction analysis promising results are obtained from the 3 lepton signature generated by the single $\tilde{\chi}_{1}^{ \pm}$production. The $\tilde{\chi}_{1}^{ \pm}$and $\tilde{\nu}_{\mu L}$ mass reconstructions can be improved using constrained fits.

In the hypothesis of a single dominant coupling constant of type $\lambda_{1 j k}^{\prime}$, exactly the same kind of $\tilde{\chi}_{1}^{0}, \tilde{\chi}_{1}^{ \pm}$and $\tilde{\nu}_{\mu}$ mass reconstructions can be performed by selecting the $e+e+\mu+2 j+\nu$ events. In contrast, the case of a single dominant $\lambda_{3 j k}^{\prime}$ coupling requires more sophisticated methods.

As a conclusion, in the case of a single dominant coupling constant of type $\lambda_{1 j k}^{\prime}$ or $\lambda_{2 j k}^{\prime}$, 


\begin{tabular}{|l|l|l|l|l|l|l|l|}
\hline$\lambda_{312}^{\prime}$ & $\lambda_{313}^{\prime}$ & $\lambda_{321}^{\prime}$ & $\lambda_{322}^{\prime}$ & $\lambda_{323}^{\prime}$ & $\lambda_{331}^{\prime}$ & $\lambda_{332}^{\prime}$ & $\lambda_{333}^{\prime}$ \\
\hline 0.13 & 0.23 & 0.18 & 0.41 & 0.70 & 0.33 & 1.17 & 2.05 \\
\hline
\end{tabular}

Table 5: Sensitivities at $95 \% C L$ on the $\lambda_{3 j k}^{\prime}$ coupling constants derived from the sensitivity on $\lambda_{311}^{\prime}$ for a luminosity of $\mathcal{L}=2 \mathrm{fb}^{-1}$ and the following set of SUSY parameters, $\tan \beta=1.5, M_{2}=200 \mathrm{GeV}$, $\mu=-200 \mathrm{GeV}$ and $m_{0}=180 \mathrm{GeV}$.

the $\tilde{\chi}_{1}^{0}, \tilde{\chi}_{1}^{ \pm}$and $\tilde{\nu}_{\mu}$ mass reconstructions based on the 3 lepton signature generated by the single $\tilde{\chi}_{1}^{ \pm}$production at Tevatron can easily give precise results, in contrast with the mass reconstructions performed in the superpartner pair production analysis at hadronic colliders which suffer a high combinatorial background [39].

\subsubsection{Model dependence of the results}

In this Section, we discuss qualitatively the impact on our results of the choice of our theoretical model, namely mSUGRA with the infrared fixed point hypothesis for the top quark Yukawa coupling. We focus on the discovery potentials obtained in Sections 4.5.1, 4.5.2 and 4.5.3, since the choice of the theoretical framework does not influence the study of the neutralino mass reconstruction made in Section 4.5.4 which is model independent.

The main effect of the infrared fixed point approach is to fix the value of the $\tan \beta$ parameter, up to the ambiguity on the low or high solution. Therefore, the infrared fixed point hypothesis has no important effects on the results since the dependences of the single gaugino productions rates on $\tan \beta$ are smooth, in the high $\tan \beta$ scenario (see Section 3.1.1).

As we have mentioned in Section 2, in the mSUGRA scenario, the $|\mu|$ parameter is fixed. This point does not influence much our results since the single gaugino production cross sections vary weakly with $|\mu|$ as shown in Section 3.1.1.

Another particularity of the mSUGRA model is that the LSP is the $\tilde{\chi}_{1}^{0}$ in most of the parameter space. For instance, in a model where the LSP would be the lightest chargino or a squark, the contribution to the three lepton signature from the $\tilde{\chi}_{1}^{ \pm} l^{\mp}$ production would vanish.

Finally in mSUGRA, the squark masses are typically larger than the lightest chargino mass so that the decay $\tilde{\chi}_{1}^{ \pm} \rightarrow \tilde{\chi}_{1}^{0} l^{ \pm} \nu$ has a branching ratio of at least $\sim 30 \%$ (see Section 4.1). In a scenario where $m_{\tilde{\chi}_{1}^{ \pm}}>m_{\tilde{q}}$, the two-body decay $\tilde{\chi}_{1}^{ \pm} \rightarrow \tilde{q} q$ would be dominant so that the contribution to the three lepton signature from the $\tilde{\chi}_{1}^{ \pm} l^{\mp}$ production would be small. Besides, in mSUGRA, the $\tilde{\chi}_{1}^{ \pm}-\tilde{\chi}_{1}^{0}$ mass difference is typically large enough to avoid significant branching ratio for the $R_{p}$ decay of the lightest chargino which would result in a decrease of the sensitivities on the SUSY parameters presented in Sections 4.5.1, 4.5.2 and 4.5.3.

In a model where the contribution to the three lepton signature from the $\tilde{\chi}^{ \pm} l^{\mp}$ production would be suppressed, the three lepton final state could be generated in a significant way by other single gaugino productions, namely the $\tilde{\chi}^{ \pm} \nu, \tilde{\chi}^{0} l^{\mp}$ or $\tilde{\chi}^{0} \nu$ productions. 


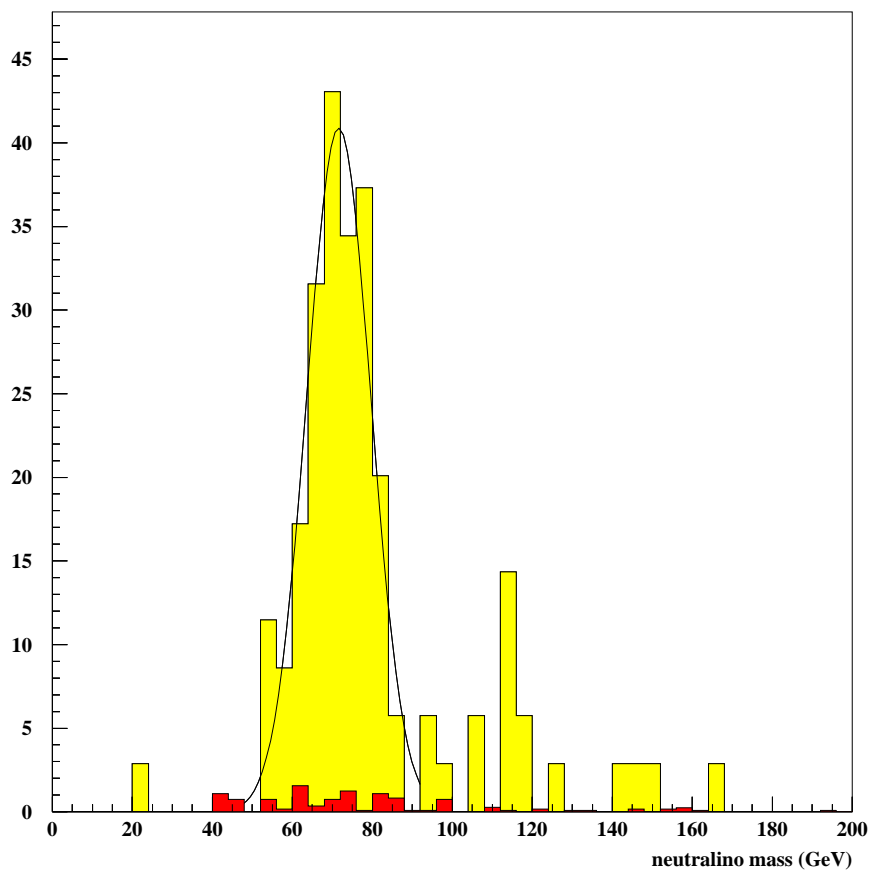

Figure 18: Distribution of the softer $\mu+2 j$ invariant mass in the $e+\mu+\mu+2 j+\nu$ events, for a luminosity of $\mathcal{L}=10 \mathrm{fb}^{-1}$. The sum of the $W Z, Z Z$ and $t \bar{t}$ backgrounds is in black and the SUSY signal is in grey. The mSUGRA point taken for this figure is $m_{0}=200 \mathrm{GeV}, M_{2}=150 \mathrm{GeV}, \tan \beta=1.5$ and $\operatorname{sign}(\mu)<0$ $\left(m_{\tilde{\chi}_{1}^{0}}=77.7 \mathrm{GeV}\right)$ and the considered $\not R_{p}$ coupling is $\lambda_{211}^{\prime}=0.09$.

\section{$5 \quad$ Like sign dilepton signature analysis}

\section{$5.1 \quad$ Signal}

Within the context of the mSUGRA model, three of the single gaugino productions via $\lambda_{i j k}^{\prime}$ presented in Section 3.1 can generate a final state containing a pair of same sign leptons. As a matter of fact, the like sign dilepton signature can be produced through the reactions $p \bar{p} \rightarrow \tilde{\chi}_{1}^{0} l_{i}^{ \pm} ; p \bar{p} \rightarrow \tilde{\chi}_{2}^{0} l_{i}^{ \pm}, \tilde{\chi}_{2}^{0} \rightarrow \tilde{\chi}_{1}^{0}+X\left(X \neq l^{ \pm}\right) ; p \bar{p} \rightarrow \tilde{\chi}_{1}^{ \pm} l_{i}^{\mp}, \tilde{\chi}_{1}^{ \pm} \rightarrow \tilde{\chi}_{1}^{0} q \bar{q}$ and $p \bar{p} \rightarrow \tilde{\chi}_{1}^{ \pm} \nu_{i}, \tilde{\chi}_{1}^{ \pm} \rightarrow \tilde{\chi}_{1}^{0} l^{ \pm} \nu, i$ corresponding to the flavour index of the $\lambda_{i j k}^{\prime}$ coupling. Indeed, since the $\tilde{\chi}_{1}^{0}$ is a Majorana particle, it decays via $\lambda_{i j k}^{\prime}$ into a lepton, as $\tilde{\chi}_{1}^{0} \rightarrow l_{i} u_{j} \bar{d}_{k}$, and into an anti-lepton, as $\tilde{\chi}_{1}^{0} \rightarrow \bar{l}_{i} \bar{u}_{j} d_{k}$, with the same probability. The $\tilde{\chi}_{3,4}^{0} l_{i}^{ \pm}, \tilde{\chi}_{2}^{ \pm} l_{i}^{\mp}$ and $\tilde{\chi}_{2}^{ \pm} \nu_{i}$ productions do not bring significant contributions to the like sign dilepton signature due to their relatively small cross sections (see Section 3.1.1).

In mSUGRA, the most important contribution to the like sign dilepton signature originates from the $\tilde{\chi}_{1}^{0} l_{i}^{ \pm}$production since this reaction has a dominant cross section in most of the mSUGRA parameter space, as shown in Section 3.1.1. The other reason is that if $\tilde{\chi}_{1}^{0}$ is the LSP, the $\tilde{\chi}_{1}^{0} l_{i}^{ \pm}$production rate is not affected by branching ratios of any cascade decay since the $\tilde{\chi}_{1}^{0}$ only decays through $R_{p}$ coupling. 

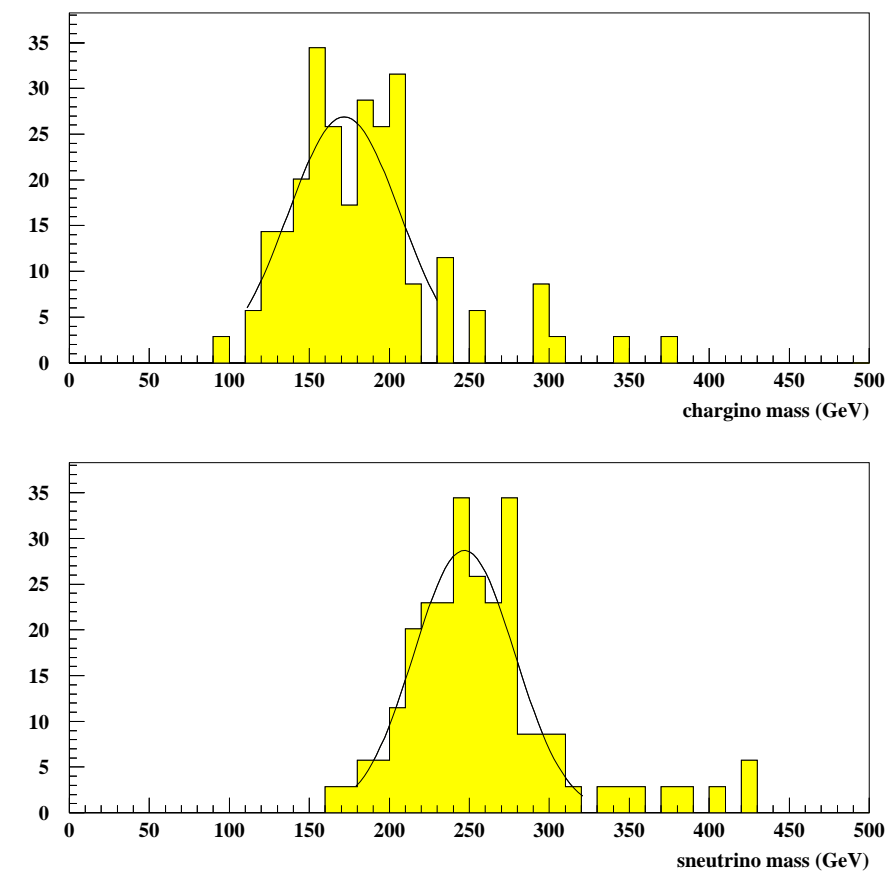

Figure 19: Distributions of the $e+$ softer $\mu+2 j+\nu$ (upper plot) and $e+\mu+\mu+2 j+\nu$ (lower plot) invariant masses in the $e+\mu+\mu+2 j+\nu$ events, for a luminosity of $\mathcal{L}=10 \mathrm{fb}^{-1}$. The mSUGRA point taken for these figures is $m_{0}=200 \mathrm{GeV}, M_{2}=150 \mathrm{GeV}, \tan \beta=1.5$ and $\operatorname{sign}(\mu)<0\left(m_{\tilde{\chi}_{1}^{ \pm}}=158.3 \mathrm{GeV}\right.$, $\left.m_{\tilde{\nu}_{\mu L}}=236 \mathrm{GeV}\right)$ and the considered $\not R_{p}$ coupling is $\lambda_{211}^{\prime}=0.09$.

\subsection{Standard Model background of the like sign dilepton signature at Teva- tron}

The $b \bar{b}$ production can lead to the like sign dilepton signature if both of the $\mathrm{b}$ quarks decay semi-leptonically. The leading order cross section of the $\bar{b} b$ production at Tevatron for an energy of $\sqrt{s}=2 \mathrm{TeV}$ is $\sigma(p \bar{p} \rightarrow b \bar{b}) \approx 4.65410^{10} \mathrm{fb}$. This rate has been calculated with PYTHIA [32] using the CTEQ2L structure function.

The $t \bar{t}$ production, followed by the decays $t \rightarrow W^{+} b \rightarrow l^{+} \nu b, \bar{t} \rightarrow W^{-} \bar{b} \rightarrow \bar{q} q \bar{b} \rightarrow$ $\bar{q} q l^{+} \nu \bar{c}$, or $t \rightarrow W^{+} b \rightarrow \bar{q} q b \rightarrow \bar{q} q l^{-} \bar{\nu} c, \bar{t} \rightarrow W^{-} \bar{b} \rightarrow l^{-} \bar{\nu} \bar{b}$, also generates a final state with two same sign leptons. The leading order cross section of the $t \bar{t}$ production at $\sqrt{s}=2 \mathrm{TeV}$, including the relevant branching ratios, is $\sigma(p \bar{p} \rightarrow t \bar{t}) \times 2 \times B\left(W \rightarrow l_{p} \nu_{p}\right) \times B\left(W \rightarrow q_{p} \bar{q}_{p^{\prime}}\right) \approx$ $3181 \mathrm{fb}(2800 \mathrm{fb})$ for $m_{\text {top }}=170 \mathrm{GeV}(175 \mathrm{GeV})$ with $p, p^{\prime}=1,2,3$.

The third important source of Standard Model background is the $t \bar{b} / \bar{t} b$ production since the (anti-) $b$ quark can undergo a semi-leptonic decay as $b \rightarrow l^{-} \bar{\nu} c\left(\bar{b} \rightarrow l^{+} \nu \bar{c}\right)$ and the (anti-) top quark can decay simultaneously as $t \rightarrow b W^{+} \rightarrow b l^{+} \nu\left(\bar{t} \rightarrow \bar{b} W^{-} \rightarrow \bar{b} l^{-} \bar{\nu}\right)$. The leading order cross section at $\sqrt{s}=2 \mathrm{TeV}$ including the branching fraction is $\sigma(p \bar{p} \rightarrow$ $t q, \bar{t} q) \times B\left(W \rightarrow l_{p} \nu_{p}\right) \approx 802 \mathrm{fb}(687 \mathrm{fb})$ for $m_{\text {top }}=170 \mathrm{GeV}(175 \mathrm{GeV})$ with $p=1,2,3$.

Other small sources of Standard Model background are the $W^{ \pm} W^{\mp}$ production, followed by the decays: $W \rightarrow l \nu$ and $W \rightarrow b u_{p}(p=1,2)$ or $W \rightarrow b u_{p}$ and $W \rightarrow b u_{p}$ $(p=1,2)$, the $W^{ \pm} Z^{0}$ production, followed by the decays: $W \rightarrow l \nu$ and $Z \rightarrow b \bar{b}$ or 
$W \rightarrow q_{p} \bar{q}_{p^{\prime}}$ and $Z \rightarrow b \bar{b}$, and the $Z^{0} Z^{0}$ production, followed by the decays: $Z \rightarrow l \bar{l}$ and $Z \rightarrow b \bar{b}$ or $Z \rightarrow q_{p} \bar{q}_{p}$ and $Z \rightarrow b \bar{b}$.

Finally, the 3 lepton final states generated by the $Z^{0} Z^{0}$ and $W^{ \pm} Z^{0}$ productions (see Section 4.2) can be mistaken for like sign dilepton events in case where one of the leptons is lost in the detection. Non-physics sources of background can also be caused by some fake leptons or by the misidentification of the charge of a lepton.

Therefore for the study of the Standard Model background associated to the like sign dilepton signal at Tevatron Run II, we consider the $b \bar{b}$, the $t \bar{t}$, the $W^{ \pm} W^{\mp}$ and the single top production and both the physics and non-physics contributions generated by the $W^{ \pm} Z^{0}$ and $Z^{0} Z^{0}$ productions.

\subsection{Supersymmetric background of the like sign dilepton signature at Teva- tron}

All the pair productions of superpartners are a source of SUSY background for the like sign dilepton signature originating from the single gaugino productions. Indeed, both of the produced superpartners initiate a cascade decay ended by the $R_{p}$ decay of the LSP through $\lambda_{i j k}^{\prime}$, and if the two LSP's undergo the same decay $\tilde{\chi}_{1}^{0} \rightarrow l_{i} u_{j} \bar{d}_{k}$ or $\tilde{\chi}_{1}^{0} \rightarrow \bar{l}_{i} \bar{u}_{j} d_{k}$, two same sign charged leptons are generated. Another possible way for the SUSY pair production to generate the like sign dilepton signature is that only one of the LSP's decays into a charged lepton of a given sign, the other decaying as $\tilde{\chi}_{1}^{0} \rightarrow \nu_{i} d_{j} d_{k}$, and a second charged lepton of the same sign is produced in the cascade decays.

The cross sections of the superpartners pair productions have been studied in Section 4.3 .

\subsection{Cuts}

In order to simulate the single chargino productions $p \bar{p} \rightarrow \tilde{\chi}_{1}^{ \pm} l^{\mp}, p \bar{p} \rightarrow \tilde{\chi}_{1}^{ \pm} \nu$ and the single neutralino production $p \bar{p} \rightarrow \tilde{\chi}_{1}^{0} l^{\mp}$ at Tevatron, the matrix elements (see Appendix A) of these processes have been implemented in a version of the SUSYGEN event generator [45] allowing the generation of $p \bar{p}$ reactions [46]. The Standard Model background $\left(W^{ \pm} W^{\mp}\right.$, $W^{ \pm} Z^{0}, Z^{0} Z^{0}, t \bar{b} / \bar{t} b, t \bar{t}$ and $b \bar{b}$ productions) has been simulated using the PYTHIA event generator [32] and the SUSY background (all SUSY particles pair productions) using the HERWIG event generator [44]. SUSYGEN, PYTHIA and HERWIG have been interfaced with the SHW detector simulation package [37] (see Section 4.4).

Several selection criteria have been applied in order to reduce the background.

First, we have selected the events containing two same sign muons. The reason is that in the like sign dilepton signature analysis we have focused on the case of a single dominant $R_{p}$ coupling constant of the type $\lambda_{2 j k}^{\prime}$. In such a scenario, the two same charge leptons generated in the $\tilde{\chi}_{1}^{0} l^{\mp}$ production, which represents the main contribution to the like sign dilepton final state (see Section 5.1), are muons (see Fig.1 and Section 5.1). This requirement that the 2 like sign leptons have the same flavour allows to reduce the Standard Model background with respect to the signal.

We require a number of jets greater or equal to two with a transverse momentum higher than $10 \mathrm{GeV}$, namely $N_{j} \geq 2$ with $P_{t}(j)>10 \mathrm{GeV}$. This jet veto reduces the non-physics backgrounds generated by the $W^{ \pm} Z^{0}$ and $Z^{0} Z^{0}$ productions (see Section 5.2) which produce at most one hard jet (see Section 4.4).

Besides, some effective cuts concerning the energies of the 2 selected muons have been applied. In Fig.20, we present the distributions of the 2 muon energies in the like sign 

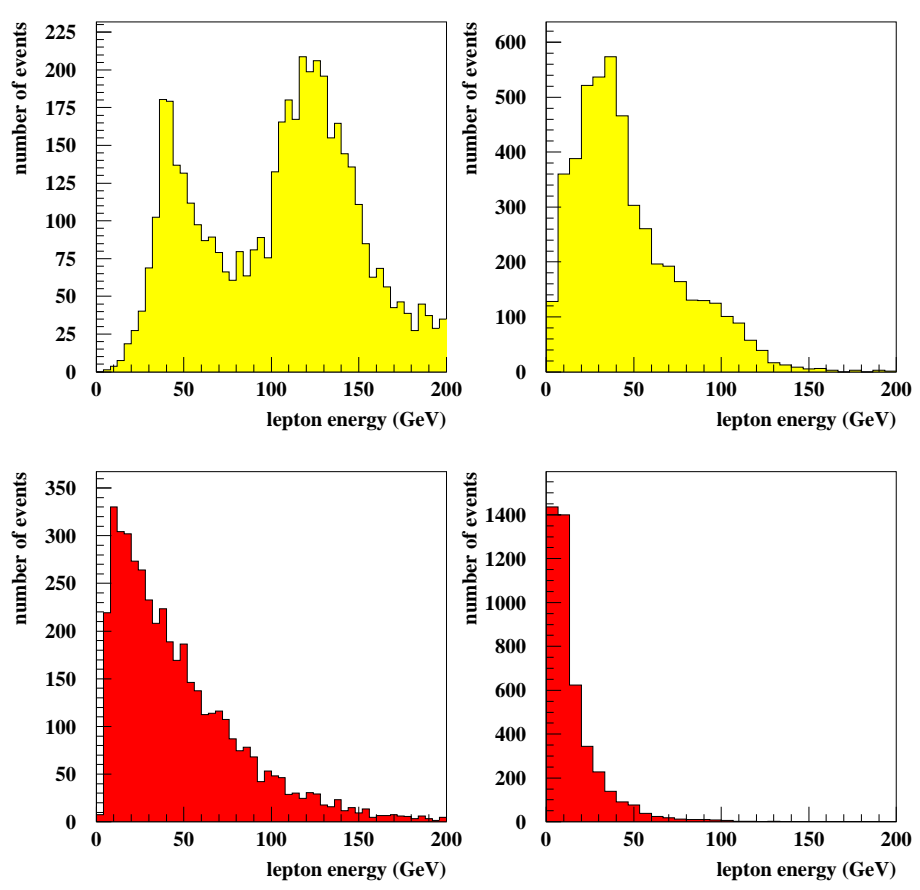

Figure 20: Distributions of the 2 muon energies (in $\mathrm{GeV}$ ) in the events containing 2 same sign muons and at least 2 jets generated by the Standard Model background (lower curve), namely the $W^{ \pm} W^{\mp}, W^{ \pm} Z^{0}, Z^{0} Z^{0}, t \bar{t}, t \bar{b} / \bar{t} b$ and b $\bar{b}$ productions, and the SUSY signal (upper curve), for $\lambda_{211}^{\prime}=0.05, M_{2}=250 \mathrm{GeV}, m_{0}=200 \mathrm{GeV}, \tan \beta=1.5$ and sign $(\mu)<0$. The left plots represent the leading muon distributions and the right plots the second leading muon distributions. The numbers of events correspond to an integrated luminosity of $\mathcal{L}=10 \mathrm{fb}^{-1}$.

dimuon events generated by the Standard Model background $\left(W^{ \pm} W^{\mp}, W^{ \pm} Z^{0}, Z^{0} Z^{0}, t \bar{t}\right.$, $t \bar{b} / \bar{t} b$ and $b \bar{b})$ and the SUSY signal. Based on these distributions, we have chosen the following cuts on the muon energies: $E\left(\mu_{2}\right)>20 \mathrm{GeV}$ and $E\left(\mu_{1}\right)>20 \mathrm{GeV}$.

We will refer to all the selection criteria described above, namely 2 same sign muons with $E\left(\mu_{2}\right)>20 \mathrm{GeV}$ and $E\left(\mu_{1}\right)>20 \mathrm{GeV}$, and $N_{j} \geq 2$ with $P_{t}(j)>10 \mathrm{GeV}$, as cut 1 .

Let us explain the origin of the two peaks in the upper left plot of Fig.20. This will be helpful for the mass reconstruction study of Section 5.5.2.

The main contribution to the like sign dimuon signature from the SUSY signal is the $\tilde{\chi}_{1}^{0} \mu^{ \pm}$ production (see Section 5.1) in the case of a single dominant $\lambda_{2 j k}^{\prime}$ coupling. Furthermore, the dominant contribution to this production is the reaction $p \bar{p} \rightarrow \tilde{\mu}_{L}^{ \pm} \rightarrow \tilde{\chi}_{1}^{0} \mu^{ \pm}$. In this reaction, the $\mu^{ \pm}$produced together with the $\tilde{\chi}_{1}^{0}$ has an energy around $E\left(\mu^{ \pm}\right) \approx$ $\left(m_{\tilde{\mu}_{L}^{ \pm}}^{2}+m_{\mu^{ \pm}}^{2}-m_{\tilde{\chi}_{1}^{0}}^{2}\right) / 2 m_{\tilde{\mu}_{L}^{ \pm}}=121.9 \mathrm{GeV}$ for the SUSY point considered in Fig.20, namely $M_{2}=250 \mathrm{GeV}, m_{0}=200 \mathrm{GeV}, \tan \beta=1.5$ and $\operatorname{sign}(\mu)<0$, which gives rise to the mass spectrum: $m_{\tilde{\chi}_{1}^{0}}=127.1 \mathrm{GeV}, m_{\tilde{\chi}_{2}^{0}}=255.3 \mathrm{GeV}, m_{\tilde{\chi}_{1}^{ \pm}}=255.3 \mathrm{GeV}, m_{\tilde{l}_{L}^{ \pm}}=298 \mathrm{GeV}$ and $m_{\tilde{\nu}_{L}^{ \pm}}=294 \mathrm{GeV}$. This energy value corresponds approximatively to the mean value of the right peak of the leading muon energy distribution presented in the upper left plot of Fig.20. This is due to the fact that the leading muon in the dimuon events generated by the reaction $p \bar{p} \rightarrow \tilde{\chi}_{1}^{0} \mu^{ \pm}$is the $\mu^{ \pm}$produced together with the $\tilde{\chi}_{1}^{0}$ for relatively important values of the $m_{\tilde{\mu}_{L}^{ \pm}}-m_{\tilde{\chi}_{1}^{0}}$ mass difference. The right peak in the upper left plot of Fig.20 


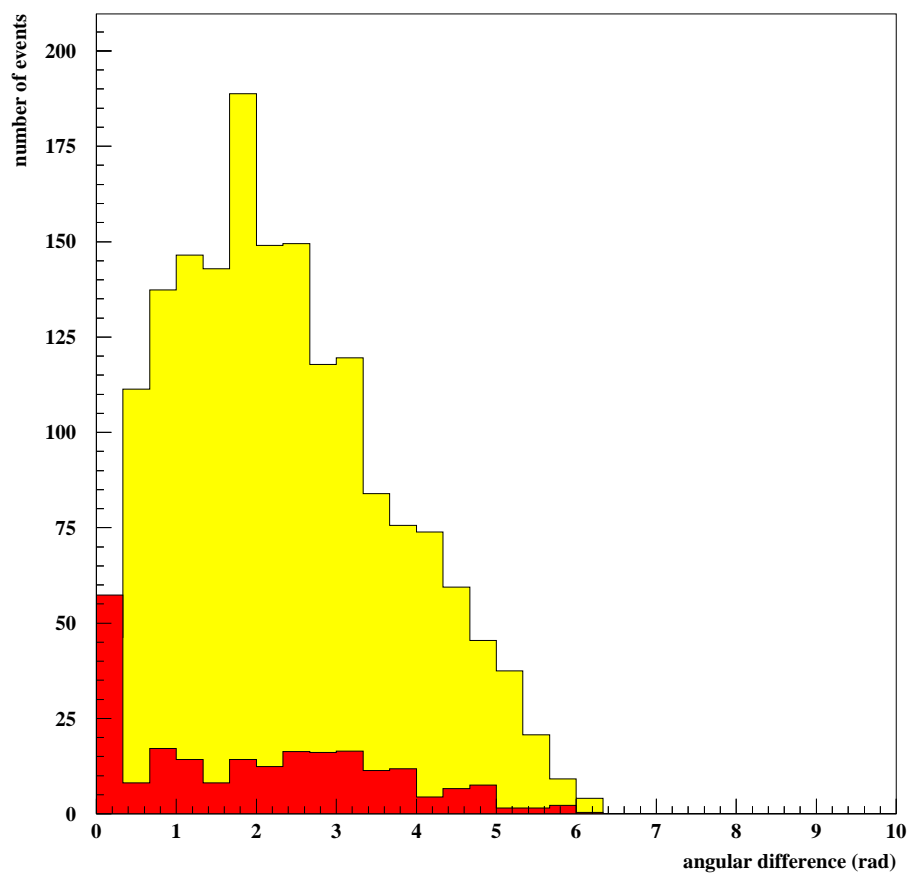

Figure 21: Distributions of the $\Delta R$ angular difference (in rad) between the second leading muon and the second leading jet in the like sign dimuons events selected by applying cut 1 and generated by the Standard Model background (curve in black), namely the $W^{ \pm} W^{\mp}, W^{ \pm} Z^{0}, Z^{0} Z^{0}, t \bar{t}, t \bar{b} / \bar{t} b$ and $b \bar{b}$ productions, and the SUSY signal (curve in grey), for $\lambda_{211}^{\prime}=0.05$, $M_{2}=250 \mathrm{GeV}, m_{0}=200 \mathrm{GeV}, \tan \beta=1.5$ and $\operatorname{sign}(\mu)<0$. The numbers of events correspond to an integrated luminosity of $\mathcal{L}=10 \mathrm{fb}^{-1}$.

is thus associated to the $\tilde{\chi}_{1}^{0} \mu^{ \pm}$production.

Similarly, the left peak in the upper left plot of Fig.20 corresponds to the reactions $p \bar{p} \rightarrow \tilde{\mu}_{L}^{ \pm} \rightarrow \tilde{\chi}_{2}^{0} \mu^{ \pm}$and $p \bar{p} \rightarrow \tilde{\nu}_{\mu L} \rightarrow \tilde{\chi}_{1}^{ \pm} \mu^{\mp}$ which produce $\mu^{ \pm}$of energies around $E\left(\mu^{ \pm}\right) \approx$ $\left(m_{\tilde{\mu}_{L}^{ \pm}}^{2}+m_{\mu^{ \pm}}^{2}-m_{\tilde{\chi}_{2}^{2}}^{2}\right) / 2 m_{\tilde{\mu}_{L}^{ \pm}}=39.6 G e V$ and $E\left(\mu^{ \pm}\right) \approx\left(m_{\tilde{\nu}_{\mu L}}^{2}+m_{\mu^{ \pm}}^{2}-m_{\tilde{\chi}_{1}^{ \pm}}^{2}\right) / 2 m_{\tilde{\nu}_{\mu L}}=$ $36.2 \mathrm{GeV}$, respectively. The $\tilde{\chi}_{1}^{ \pm} \nu_{\mu}$ production represents a less important contribution to the like sign dimuon events compared to the 3 above single gaugino productions since the 2 same sign leptons generated in this production are not systematically muons and the involved branching ratios have smaller values (see Section 5.1).

Finally, since the leptons produced in the quark $b$ decays are not well isolated (as in the $W^{ \pm} W^{\mp}, W^{ \pm} Z^{0}, Z^{0} Z^{0}, t \bar{t}, t \bar{b} / \bar{t} b$ and $b \bar{b}$ productions), we have applied some cuts on the lepton isolation. We have imposed the isolation cut $\Delta R=\sqrt{\delta \phi^{2}+\delta \theta^{2}}>0.4$ where $\phi$ is the azimuthal angle and $\theta$ the polar angle between the 2 same sign muons and the 2 hardest jets. This cut is for example motivated by the distributions shown in Fig.21 of the $\Delta R$ angular difference between the second leading muon and the second leading jet, in the like sign dimuons events generated by the SUSY signal and Standard Model background. We call cut $\Delta R>0.4$ together with cut 1 , cut 2 .

In order to eliminate poorly isolated muons, we have also imposed that $E<2 G e V$, where $E$ represents the summed energies of the jets being close to a muon, namely the jets contained in the cone centered on a muon and defined by $\Delta R<0.25$. This cut is 

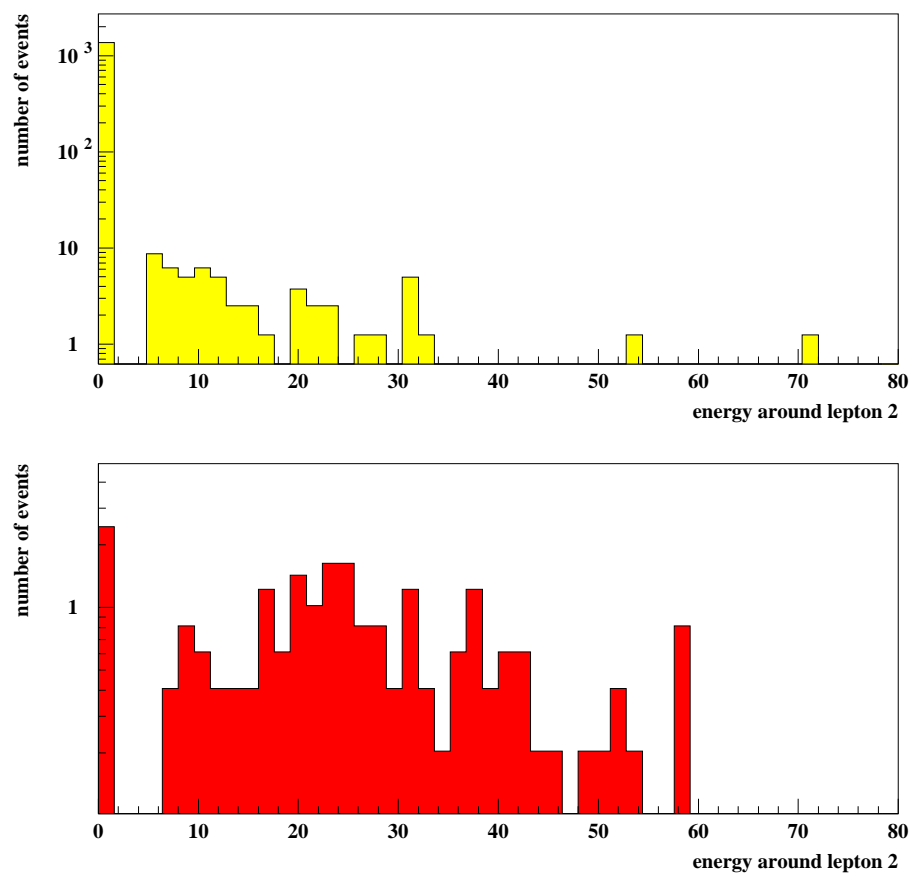

Figure 22: Distributions of the summed energies ( $E$, in $G e V$ ) of the jets being close to the second leading muon, namely the jets contained in the cone centered on the second leading muon and defined by $\Delta R<0.25$, in the like sign dimuons events selected by applying cut 2 and generated by the Standard Model background (lower curve), namely the $W^{ \pm} W^{\mp}, W^{ \pm} Z^{0}$, $Z^{0} Z^{0}, t \bar{t}, t \bar{b} / \bar{t} b$ and $b \bar{b}$ productions, and the SUSY signal (upper curve), for $\lambda_{211}^{\prime}=0.05, M_{2}=250 G e V, m_{0}=200 G e V$, $\tan \beta=1.5$ and $\operatorname{sign}(\mu)<0$. These distributions were obtained after cut $E<2 G e V$, where $E$ represents the summed energies of the jets being close to the leading muon, has been applied in these like sign dimuons events. The numbers of events correspond to an integrated luminosity of $\mathcal{L}=10 \mathrm{fb}^{-1}$.

for instance motivated by the distributions shown in Fig. 22 which represent the summed energies $E$ of the jets being close to the second leading muon in the like sign dimuons events generated by the SUSY signal and Standard Model background. We denote cut $E<2 G e V$ plus cut 2 as cut 3 .

The selected events require high energy charged leptons and jets and can thus be easily triggered at Tevatron. Moreover, the considered charged leptons and jets are typically emitted at intermediate polar angles and would thus be often detected at Tevatron. These points are illustrated in Fig. 23 where are shown the energy and polar angle distributions of the leading muon and the leading jet in the like sign dimuons events selected by applying cut 3 and generated by the SUSY signal and Standard Model background.

In Table 6, we give the numbers of like sign dilepton events expected from the Standard Model background at Tevatron Run II with the various cuts described above. We see in Table 6 that the main source of Standard Model background to the like sign dilepton signature at Tevatron is the $t \bar{t}$ production. This is due to its important cross section compared to the other Standard Model backgrounds (see Section 5.2) and to the fact that in the $t \bar{t}$ background, in contrast with the $b \bar{b}$ background, only one charged lepton of the final state is produced in a $b$-jet and is thus not isolated.

In Table 7 , we give the number of like sign dilepton events generated by the SUSY 

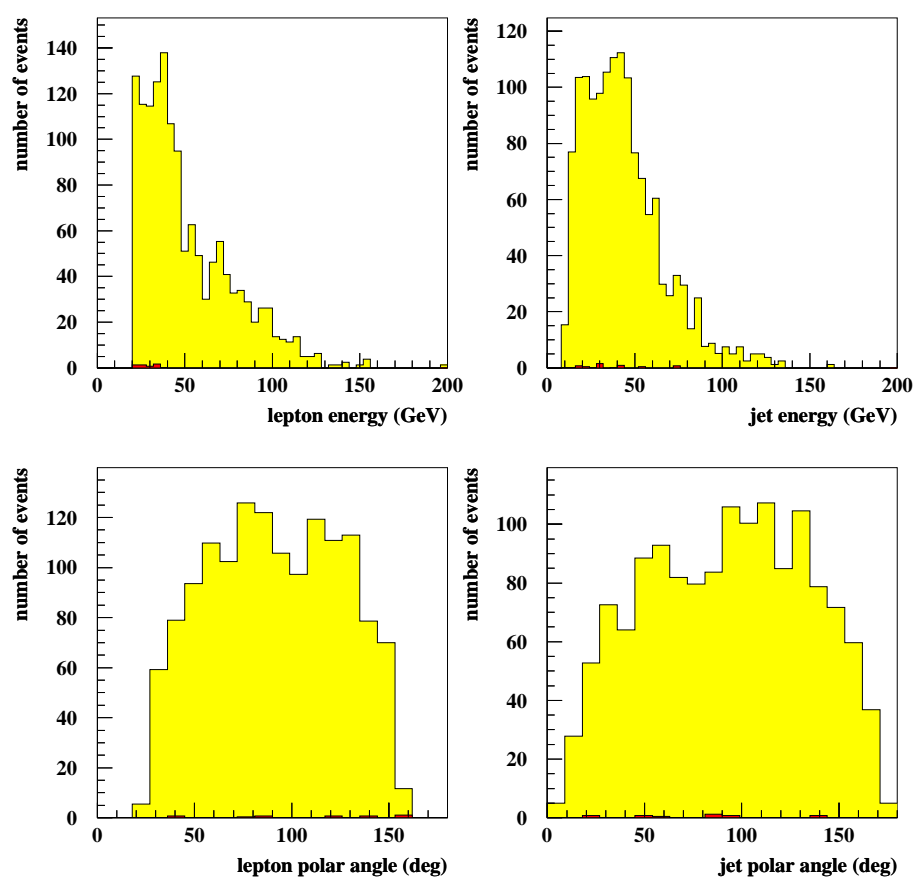

Figure 23: Energy (in $\mathrm{GeV}$ ) and polar angle ( $\theta$, in deg) distributions of the leading muon and the leading jet in the like sign dimuon events selected by applying cut 3 and generated by the Standard Model background (curve in black), namely the $W^{ \pm} W^{\mp}, W^{ \pm} Z^{0}, Z^{0} Z^{0}, t \bar{t}, t \bar{b} / \bar{t} b$ and $b \bar{b}$ productions, and the SUSY signal (curve in grey), for $\lambda_{211}^{\prime}=0.05, M_{2}=250 G e V$, $m_{0}=200 \mathrm{GeV}, \tan \beta=1.5$ and $\operatorname{sign}(\mu)<0$. The numbers of events correspond to an integrated luminosity of $\mathcal{L}=10 \mathrm{fb}^{-1}$.

background (all superpartners pair productions) at Tevatron Run II as a function of the $m_{0}$ and $m_{1 / 2}$ parameters for cut 3 . This number of events decreases as $m_{0}$ and $m_{1 / 2}$ increase due to the behaviour of the summed superpartners pair production cross section in the SUSY parameter space (see Section 4.3).

\subsection{Results}

\subsubsection{Discovery potential}

We first present the reach in the mSUGRA parameter space obtained from the analysis of the like sign dilepton final state at Tevatron Run II produced by the single neutralino and chargino productions via $\lambda_{211}^{\prime}: p \bar{p} \rightarrow \tilde{\chi}_{1,2}^{0} \mu^{ \pm}, p \bar{p} \rightarrow \tilde{\chi}_{1}^{ \pm} \mu^{\mp}$ and $p \bar{p} \rightarrow \tilde{\chi}_{1}^{ \pm} \nu_{\mu}$. The sensitivities that can be obtained on the $\lambda_{2 j k}^{\prime}$ ( $j$ and $k$ being not equal to 1 simultaneously), $\lambda_{1 j k}^{\prime}$ and $\lambda_{3 j k}^{\prime}$ coupling constants will be discussed at the end of this section.

In Fig.24, we present the $3 \sigma$ and $5 \sigma$ discovery contours and the limits at $95 \%$ confidence level in the plane $m_{0}$ versus $m_{1 / 2}$, for $\operatorname{sign}(\mu)<0, \tan \beta=1.5, \lambda_{211}^{\prime}=0.05$ and using a set of values for the luminosity. Those discovery potentials were obtained by considering the $\tilde{\chi}_{1,2}^{0} \mu^{ \pm}, \tilde{\chi}_{1}^{ \pm} \mu^{\mp}$ and $\tilde{\chi}_{1}^{ \pm} \nu_{\mu}$ productions and the background originating from the Standard Model. The signal and background were selected by using cut 3 described in Section 5.4. The reduction of the sensitivity on $m_{1 / 2}$ observed in Fig.24 as $m_{0}$ increases is due to the decrease of the $\tilde{\chi}_{1,2}^{0} \mu^{ \pm}, \tilde{\chi}_{1}^{ \pm} \mu^{\mp}$ and $\tilde{\chi}_{1}^{ \pm} \nu_{\mu}$ productions cross sections with the $m_{0}$ increase 


\begin{tabular}{|c|c|c|c|c|c|}
\hline & $W^{ \pm} Z^{0}$ & $Z^{0} Z^{0}$ & $t \bar{t}$ & $t b / \bar{t} b$ & Total \\
\hline cut 1 & $0.21 \pm 0.06$ & $0.11 \pm 0.04$ & $21.80 \pm 0.70$ & $0.69 \pm 0.13$ & $22.81 \pm 0.71$ \\
\hline cut 2 & $0.05 \pm 0.03$ & $0.03 \pm 0.03$ & $8.80 \pm 0.50$ & $0.28 \pm 0.08$ & $9.16 \pm 0.51$ \\
\hline cut 3 & $0.03 \pm 0.03$ & $0.01 \pm 0.02$ & $0.64 \pm 0.13$ & $0.10 \pm 0.05$ & $0.78 \pm 0.14$ \\
\hline
\end{tabular}

Table 6: Numbers of like sign dilepton events generated by the Standard Model background $\left(W^{ \pm} W^{\mp}\right.$, $W^{ \pm} Z^{0}, Z^{0} Z^{0}, t \bar{t}, t \bar{b} / \bar{t} b$ and $b \bar{b}$ productions) at Tevatron Run II for the cuts described in the text, assuming an integrated luminosity of $\mathcal{L}=1 \mathrm{fb}^{-1}$ and a center of mass energy of $\sqrt{\mathrm{s}}=2 \mathrm{TeV}$. The numbers of events coming from the $W^{ \pm} W^{\mp}$ and $b \bar{b}$ backgrounds have been found to be negligible after cut 3 is applied. These results have been obtained by generating $210^{4}$ events for the $W^{ \pm} Z^{0}$ production, $10^{4}$ events for the $W^{ \pm} Z^{0}$ (non-physics contribution), $310^{4}$ events for the $Z^{0} Z^{0}, 10^{4}$ events for the $Z^{0} Z^{0}$ (non-physics contribution), $310^{5}$ events for the $t \bar{t}$ and $10^{5}$ events for the $t \bar{b} / \bar{t} b$.

\begin{tabular}{|c|c|c|c|c|c|}
\hline$m_{1 / 2} \backslash m_{0}$ & $100 \mathrm{GeV}$ & $200 \mathrm{GeV}$ & $300 \mathrm{GeV}$ & $400 \mathrm{GeV}$ & $500 \mathrm{GeV}$ \\
\hline $100 \mathrm{GeV}$ & 101.64 & 54.92 & 44.82 & 39.26 & 38.77 \\
\hline $200 \mathrm{GeV}$ & 3.74 & 4.08 & 4.33 & 4.56 & 4.99 \\
\hline $300 \mathrm{GeV}$ & 1.04 & 0.63 & 0.61 & 0.70 & 0.66 \\
\hline
\end{tabular}

Table 7: Number of like sign dilepton events generated by the SUSY background (all superpartner pair productions) at Tevatron Run II as a function of the $m_{0}$ and $m_{1 / 2}$ parameters for $\tan \beta=1.5, \operatorname{sign}(\mu)<0$ and $\lambda_{211}^{\prime}=0.05$. Cut 3 (see text) has been applied. These results have been obtained by generating 7500 events and correspond to an integrated luminosity of $\mathcal{L}=1 \mathrm{fb}^{-1}$ and a center of mass energy of $\sqrt{s}=2 T e V$.

observed in Fig.5 and Fig.6. In Fig.24, we also see that the sensitivity on $m_{1 / 2}$ is reduced in the domain $m_{0} \lesssim 200 \mathrm{GeV}$. This reduction of the sensitivity is due to the fact that in mSUGRA at low $\tan \beta$ and for large values of $m_{1 / 2}$ and small values of $m_{0}$, the LSP is the Right slepton $\tilde{l}_{i R}^{ \pm}(i=1,2,3)$. Therefore, in this mSUGRA region the dominant decay channel of the lightest neutralino is $\tilde{\chi}_{1}^{0} \rightarrow \tilde{l}_{i R}^{ \pm} l_{i}^{\mp}(i=1,2,3)$ so that the $\tilde{\chi}_{1}^{0} \mu^{ \pm}$production, which is the main contribution to the like sign dilepton signature, leads to the $2 \mu^{ \pm}+2$ jets final state only in a few cases. There are two reasons. First, in this mSUGRA scenario the charged lepton produced in the main $\tilde{\chi}_{1}^{0}$ decay is not systematically a muon. Secondly, if the LSP is the Right slepton $\tilde{l}_{i R}^{ \pm}$it cannot decay in the case of a single dominant $\lambda_{i j k}^{\prime}$ coupling constant and it is thus a stable particle.

The sensitivities presented in the discovery reach of Fig.24 which are obtained from the like sign dilepton signature analysis are higher than the sensitivities shown in Fig.10 which correspond to the trilepton final state analysis. This is due to the 3 following points. First, the rate of the $\tilde{\chi}_{1}^{0} \mu^{ \pm}$production (recall that it represents the main contribution to the like sign dilepton final state) is larger than the $\sigma\left(p \bar{p} \rightarrow \tilde{\chi}_{1}^{ \pm} \mu^{\mp}\right)$ cross section in most of the mSUGRA parameter space (see Section 3.1.1). Secondly, the $\tilde{\chi}_{1}^{0}$ decay leading to the like sign dilepton final state in the case of the $\tilde{\chi}_{1}^{0} \mu^{ \pm}$production has a larger branching ratio than the cascade decay initiated by the $\tilde{\chi}_{1}^{ \pm}$which generates the trilepton final state (see Sections 4.1 and 5.1). Finally, at Tevatron Run II the Standard Model background of the like sign dilepton signature is weaker than the trilepton Standard Model background (see Tables 3 and 7).

It is clear from Fig.24 that at low values of the $m_{0}$ and $m_{1 / 2}$ parameters, high sensitivi- 

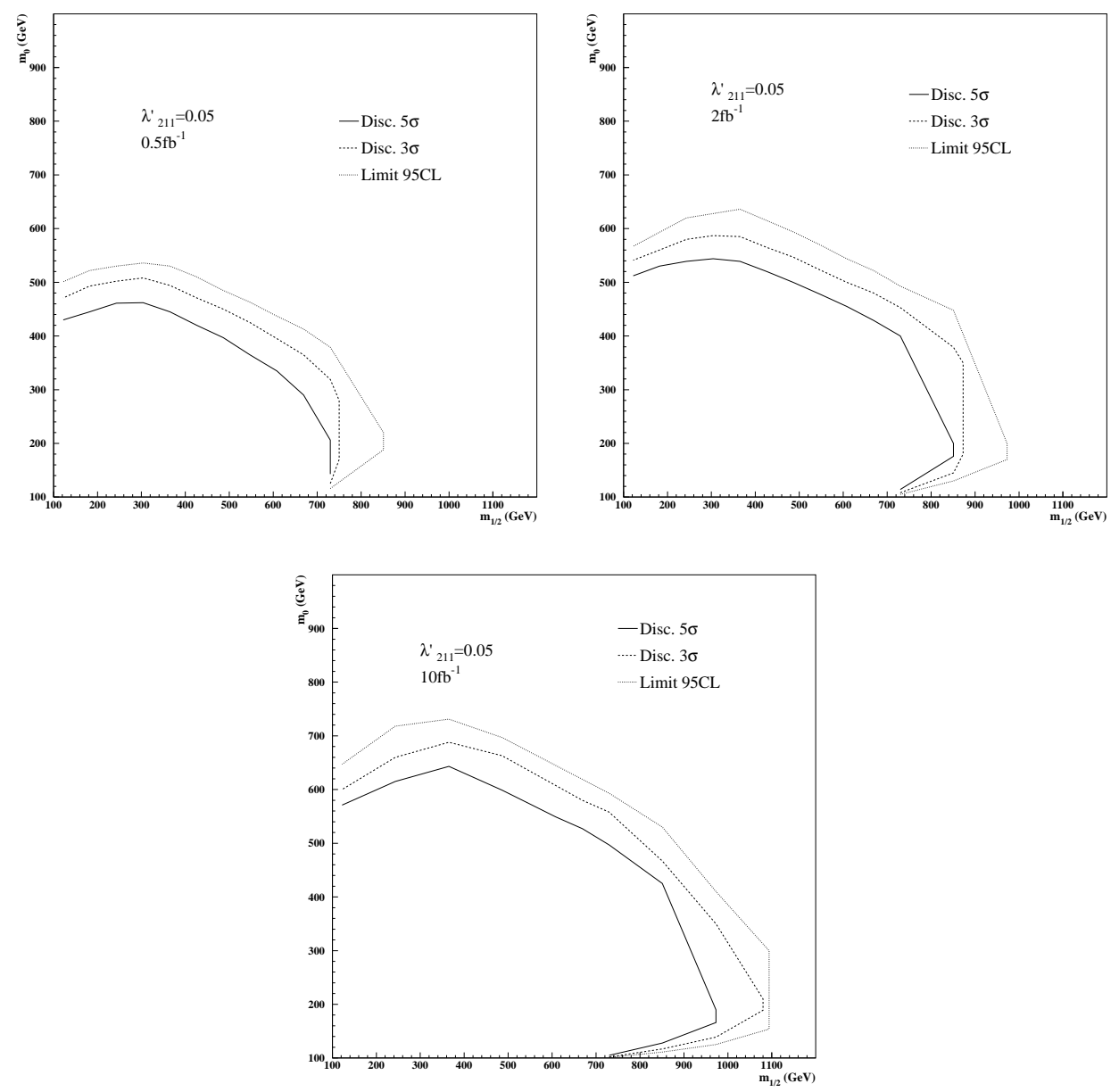

Figure 24: Discovery contours at $5 \sigma$ (full line), $3 \sigma$ (dashed line) and limit at 95\% C.L. (dotted line) obtained from the like sign dilepton signature analysis at Tevatron Run II assuming a center of mass energy of $\sqrt{s}=2 \mathrm{TeV}$. These discovery potentials are presented in the plane $m_{0}$ versus $m_{1 / 2}$, for $\operatorname{sign}(\mu)<0, \tan \beta=1.5, \lambda_{211}^{\prime}=0.05$ and different values of luminosity.

ties can be obtained on the $\lambda_{211}^{\prime}$ coupling constant. We have found that for instance at the mSUGRA point defined as $m_{0}=200 \mathrm{GeV}, m_{1 / 2}=200 \mathrm{GeV}$, $\operatorname{sign}(\mu)<0$ and $\tan \beta=1.5$, $\lambda_{211}^{\prime}$ values of $\sim 0.03$ can be probed through the like sign dilepton analysis at Tevatron Run II assuming a luminosity of $\mathcal{L}=1 \mathrm{fb}^{-1}$. This result was obtained by applying cut 3 described in Section 5.4 on the SUSY signal $\left(\tilde{\chi}_{1,2}^{0} \mu^{ \pm}, \tilde{\chi}_{1}^{ \pm} \mu^{\mp}\right.$ and $\tilde{\chi}_{1}^{ \pm} \nu_{\mu}$ productions) and the Standard Model background.

We expect that, as in the three lepton signature analysis, interesting sensitivities could be obtained on other $\lambda_{2 j k}^{\prime}$ coupling constants.

The sensitivities obtained on the $\lambda_{3 j k}^{\prime}$ couplings from the like sign dilepton signature analysis should be weaker than the sensitivities on the $\lambda_{2 j k}^{\prime}$ couplings deduced from the same study. Indeed, in the case of a single dominant $\lambda_{3 j k}^{\prime}$ coupling the same sign leptons generated by the $\tilde{\chi}_{1}^{0} \tau^{ \pm}$production would be 2 tau leptons (see Fig.1(d) and Section 5.1). Therefore, the like sign dileptons $\left(e^{ \pm} e^{ \pm}\right.$or $\mu^{ \pm} \mu^{ \pm}$) produced by the $R_{p}$ signal would be mainly generated in tau decays and would thus have higher probabilities to not pass the analysis cuts on the particle energy. Moreover, the requirement of $e^{ \pm} e^{ \pm}$or $\mu^{ \pm} \mu^{ \pm}$events 
would decrease the efficiency after cuts of the $R_{p}$ signal due to the hadronic decay of the tau. Finally, the selection of two same flavour like sign dileptons $\left(e^{ \pm} e^{ \pm}\right.$or $\left.\mu^{ \pm} \mu^{ \pm}\right)$would reduce the $R_{p}$ signal, since each of the 2 produced taus could decay either into an electron or a muon, and hence would not be an effective cut anymore.

The sensitivities obtained on the $\lambda_{1 j k}^{\prime}$ couplings from the like sign dilepton signature study are expected to be identical to the sensitivities on the $\lambda_{2 j k}^{\prime}$ couplings obtained from the same study. Indeed, in the case of a single dominant $\lambda_{1 j k}^{\prime}$ coupling constant, the only difference in the like sign dilepton signature analysis would be that $e^{ \pm} e^{ \pm}$events should be selected instead of $\mu^{ \pm} \mu^{ \pm}$events (see Fig.1(d) and Section 5.1). Nevertheless, a smaller number of $\lambda_{1 j k}^{\prime}$ couplings is expected to be probed since the low-energy constraints on the $\lambda_{1 j k}^{\prime}$ couplings are generally stronger than the limits on the $\lambda_{2 j k}^{\prime}$ couplings [4].

In the high $\tan \beta$ case, the lightest stau $\tilde{\tau}_{1}$ can become the LSP instead of the lightest neutralino, due to a large mixing in the third generation of charged sleptons. In such a situation, the dominant decay channel of the lightest neutralino is $\tilde{\chi}_{1}^{0} \rightarrow \tilde{\tau}_{1}^{ \pm} \tau^{\mp}$. Two scenarios must then be discussed: if the single dominant $R_{p}$ coupling is not of the type $\lambda_{3 j k}^{\prime}$, the $\tilde{\tau}_{1}^{ \pm}$-LSP is a stable particle so that the reaction $p \bar{p} \rightarrow \tilde{\chi}_{1}^{0} l_{i}^{ \pm}$, representing the main contribution to the like sign dilepton final state, does not often lead to the $2 \mu^{ \pm}+2$ jets signature. If the single dominant $R_{p}$ coupling is of the type $\lambda_{3 j k}^{\prime}$, the $\tilde{\chi}_{1}^{0} \tau^{ \pm}$production can receive a contribution from the resonant $\tilde{\tau}_{2}^{ \pm}$production (see Fig. $1(\mathrm{~d})$ ) and the $\tilde{\tau}_{1}^{ \pm}$-LSP decays via $\lambda_{3 j k}^{\prime}$ as $\tilde{\tau}_{1}^{ \pm} \rightarrow u_{j} d_{k}$ so that the $2 \mu^{ \pm}+2$ jets signature can still be generated in a significant way by the $p \bar{p} \rightarrow \tilde{\chi}_{1}^{0} \tau^{ \pm}$reaction.

We end this Section by some comments on the effect of the supersymmetric $R_{p}$ conserving background to the like sign dilepton signature. In order to illustrate this discussion, we consider the results on the $\lambda_{211}^{\prime}$ coupling constant.

We see from Table 7 that the SUSY background to the like sign dilepton final state can affect the sensitivity on the $\lambda_{211}^{\prime}$ coupling constant obtained by considering only the Standard Model background, which is shown in Fig.24, only in the region of small superpartners masses, namely in the domain $m_{1 / 2} \lesssim 300 \mathrm{GeV}$ for $\tan \beta=1.5, \operatorname{sign}(\mu)<0$ and assuming a luminosity of $\mathcal{L}=1 \mathrm{fb}^{-1}$.

In contrast with the SUSY signal amplitude which is increased if $\lambda_{211}^{\prime}$ is enhanced, the SUSY background amplitude is typically independent on the value of the $\lambda_{211}^{\prime}$ coupling constant since the superpartner pair production does not involve $R_{p}$ couplings. Therefore, even if we consider the SUSY background in addition to the Standard Model one, it is still true that large values of the $\lambda_{211}^{\prime}$ coupling can be probed over a wider domain of the SUSY parameter space than low values, as can be observed in Fig. 24 for $m_{1 / 2} \gtrsim 300 \mathrm{GeV}$. Note that in Fig.24 larger values of $\lambda_{211}^{\prime}$ still respecting the indirect limit could have been considered.

Finally, we mention that further cuts, as for instance some cuts based on the superpartners mass reconstructions (see Section 5.5.2), could allow to reduce the SUSY background to the like sign dilepton signature.

\subsubsection{Mass reconstructions}

The $\tilde{\chi}_{1}^{0}$ and $\tilde{l}_{L}^{ \pm}$mass reconstructions can be performed in a model independent way via the like sign dilepton analysis. We have simulated these mass reconstructions based on the like sign dimuon events generated in the scenario of a single dominant $\lambda_{2 j k}^{\prime}$ coupling constant. In this scenario, the main SUSY contribution to the like sign dilepton signature, namely the $\tilde{\chi}_{1}^{0} \mu^{ \pm}$production, has the final state $\mu^{ \pm}+\mu^{ \pm}+2$ jets (see Section 5.1). Indeed, the 

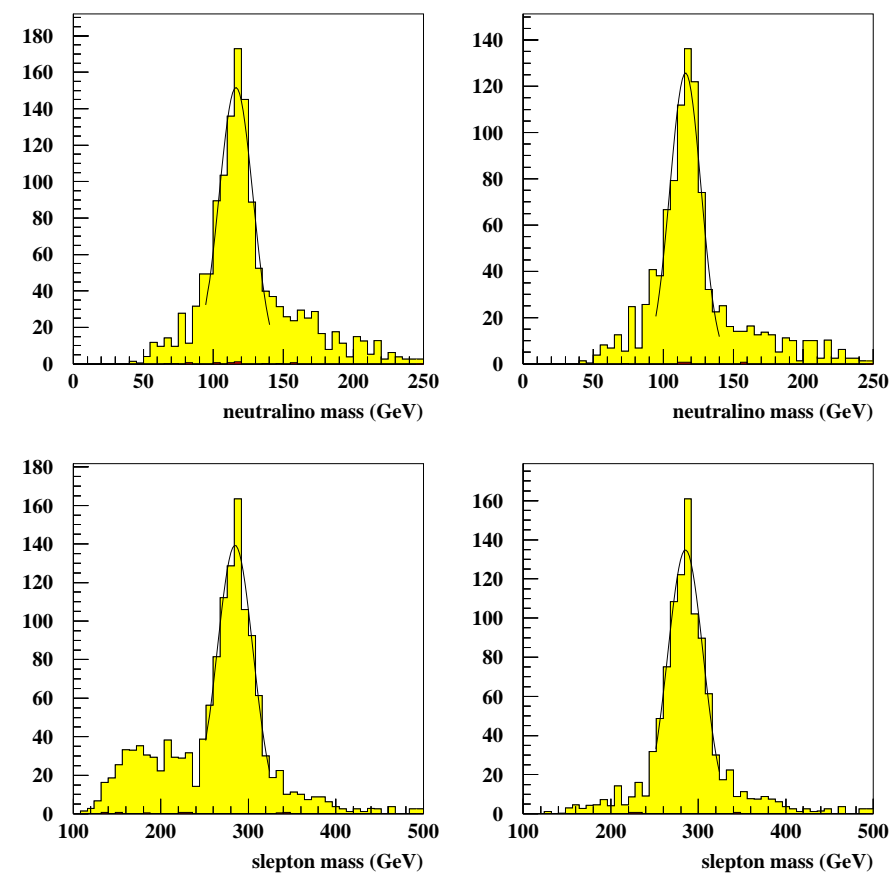

Figure 25: Distributions of the softer $\mu^{ \pm}+2$ leading jets (upper plots) and $\mu^{ \pm}+\mu^{ \pm}+2$ leading jets (lower plots) invariant masses in the $\mu^{ \pm}+\mu^{ \pm}+$jets $+\not \mathcal{E}$ events generated by the SUSY signal $\left(\tilde{\chi}_{1,2}^{0} \mu^{ \pm}\right.$, $\tilde{\chi}_{1}^{ \pm} \mu^{\mp}$ and $\tilde{\chi}_{1}^{ \pm} \nu_{\mu}$ productions), for a luminosity of $\mathcal{L}=10 \mathrm{fb}^{-1}$. The 2 right plots are obtained by applying a cut in the upper left plot of Fig.20 selecting only the peak associated to the $\tilde{\chi}_{1}^{0} \mu^{ \pm}$production. The mSUGRA point taken for this figure is, $m_{0}=200 \mathrm{GeV}, M_{2}=250 \mathrm{GeV}, \tan \beta=1.5$ and $\operatorname{sign}(\mu)<0$ $\left(m_{\tilde{\chi}_{1}^{0}}=127.1 \mathrm{GeV}, m_{\tilde{\mu}_{L}^{ \pm}}=298.0 \mathrm{GeV}\right)$ and the considered $\not R_{p}$ coupling is $\lambda_{211}^{\prime}=0.05$.

produced $\tilde{\chi}_{1}^{0}$ decays into $\mu^{ \pm} u_{j} d_{k}$ through $\lambda_{2 j k}^{\prime}$. The muon generated together with the $\tilde{\chi}_{1}^{0}$ can be identified as the leading muon for relatively large $m_{\tilde{\mu}_{L}^{ \pm}}-m_{\tilde{\chi}_{1}^{0}}$ mass differences (see Section 5.4). Note that for nearly degenerate values of $m_{\tilde{\mu}_{L}^{ \pm}}$and $m_{\tilde{\chi}_{1}^{0}}$ the $\tilde{\chi}_{1}^{0} \mu^{ \pm}$production rate and thus the sensitivity on the SUSY parameters would be reduced (see Section 3.1.1). The muon created in the $\tilde{\chi}_{1}^{0}$ decay can thus be identified as the softer muon so that the $\tilde{\chi}_{1}^{0}$ can be reconstructed from the the softer muon and the 2 jets present in the $\tilde{\chi}_{1}^{0} \mu^{ \pm}$production final state. The other contributions to the like sign dimuons events can lead to some missing energy and at most 4 jets in the final state (see Section 5.1). Hence, we have chosen to reconstruct the $\tilde{\chi}_{1}^{0}$ from the 2 leading jets when the final state contains more than 2 jets. Once the $\tilde{\chi}_{1}^{0}$ has been reconstructed, the $\tilde{\mu}_{L}^{ \pm}$has been reconstructed from the $\tilde{\chi}_{1}^{0}$ and the leading muon since the dominant contribution to the $\tilde{\chi}_{1}^{0} \mu^{ \pm}$production is the reaction $p \bar{p} \rightarrow \tilde{\mu}_{L}^{ \pm} \rightarrow \tilde{\chi}_{1}^{0} \mu^{ \pm}$. These mass reconstructions are represented in Fig.25. In this figure, we also represent the same mass reconstructions obtained by applying a cut in the upper left plot of Fig.20 excluding the peak associated to the $\tilde{\chi}_{2}^{0} \mu^{ \pm}$and $\tilde{\chi}_{1}^{ \pm} \mu^{\mp}$ productions (see Section 5.4). The interest of this cut, as can be seen in Fig.25, is to select the $\tilde{\chi}_{1}^{0} \mu^{ \pm}$production and thus to improve the accuracy on the $\tilde{\chi}_{1}^{0}$ and $\tilde{\mu}_{L}^{ \pm}$reconstructions which are based on this production. We observe in Fig. 25 that the $\tilde{\chi}_{1}^{0}$ reconstruction has less combinatorial background than the $\tilde{\mu}_{L}^{ \pm}$reconstruction. This comes from the fact that 
the selection of the softer muon and the 2 leading jets allows to reconstruct the $\tilde{\chi}_{1}^{0}$ even in the dimuon events generated by the $\tilde{\chi}_{2}^{0} \mu^{ \pm}$and $\tilde{\chi}_{1}^{ \pm} \mu^{\mp}$ productions, while the selection of the 2 muons and the 2 leading jets does not allow to reconstruct the $\tilde{\mu}_{L}^{ \pm}$in the dimuon events generated by the $\tilde{\chi}_{2}^{0} \mu^{ \pm}$and $\tilde{\chi}_{1}^{ \pm} \mu^{\mp}$ productions (see Section 5.1). We have represented on the plots of Fig. 25 the fits of the invariant mass distributions. We see from these fits that the distributions are well peaked around the $\tilde{\chi}_{1}^{0}$ and $\tilde{\mu}_{L}^{ \pm}$generated masses. The average reconstructed masses are $m_{\tilde{\chi}_{1}^{0}}=116 \pm 11 \mathrm{GeV}$ and $m_{\tilde{\mu}_{L}^{ \pm}}=285 \pm 20 \mathrm{GeV}$.

We note that the accuracy on the $\tilde{\chi}_{1}^{0}$ (and thus on the $\tilde{\mu}_{L}^{ \pm}$) mass reconstruction could be improved if the distributions in the upper plots of Fig. 25 were recalculated by selecting the muon giving the $\tilde{\chi}_{1}^{0}$ mass the closer to the mean value of the peak obtained in the relevant upper plot of Fig.25.

In the hypothesis of a single dominant coupling constant of type $\lambda_{1 j k}^{\prime}$ or $\lambda_{3 j k}^{\prime}$, exactly the same kind of $\tilde{\chi}_{1}^{0}$ and $\tilde{\mu}_{L}^{ \pm}$mass reconstructions can be performed by selecting the $e^{ \pm}+e^{ \pm}+j e t s+\not f$ or $l_{i}^{ \pm}+l_{j}^{ \pm}+j e t s+\not$ events, respectively.

As a conclusion, the $\tilde{\chi}_{1}^{0}$ and $\tilde{\mu}_{L}^{ \pm}$mass reconstructions based on the like sign dilepton signature generated by the $\tilde{\chi}_{1,2}^{0} \mu^{ \pm}, \tilde{\chi}_{1}^{ \pm} \mu^{\mp}$ and $\tilde{\chi}_{1}^{ \pm} \nu_{\mu}$ productions at Tevatron can easily give precise results, in contrast with the mass reconstructions performed in the superpartner pair production analysis at hadronic colliders which suffer an high combinatorial background [39].

\subsubsection{Model dependence of the results}

In our theoretical framework (see Section 2), the values of the $|\mu|$ and $\tan \beta$ (up to the ambiguity of low/high solution) parameters are predicted. This has no important effects on the results presented in Sections 5.5.1 as the single gaugino production cross sections vary weakly with these parameters (see Section 3.1.1).

However, since we have worked within the mSUGRA model, the $\tilde{l}_{L}^{ \pm}$mass was typically larger than the $\tilde{\chi}_{1}^{0}$ mass. In a situation where $m_{\tilde{l}_{L}^{ \pm}}$would approach $m_{\tilde{\chi}_{1}^{0}}$, the rate of the $\tilde{\chi}_{1}^{0} l_{i}^{ \pm}$production, representing in mSUGRA the main contribution to the like sign dilepton signature (see Section 5.1), would decrease. Therefore, within a model allowing degenerate $\tilde{l}_{L}^{ \pm}$and $\tilde{\chi}_{1}^{0}$ masses or even a $\tilde{l}_{L}^{ \pm}$lighter than the $\tilde{\chi}_{1}^{0}$, other single gaugino productions than the $p \bar{p} \rightarrow \tilde{\chi}_{1}^{0} l_{i}^{ \pm}$reaction could represent the major contribution to the like sign dilepton signature in some parts of the SUSY parameter space.

Besides, in a situation where the LSP would not be the $\tilde{\chi}_{1}^{0}$, the branching ratios of the $\tilde{\chi}_{1}^{0}$ decays violating $R_{p}$ would be reduced with respect to the case where the LSP is the $\tilde{\chi}_{1}^{0}$, as often occurs in mSUGRA. However, in such a situation, the like sign dilepton signature could receive a significant contribution from a decay of the $\tilde{\chi}_{1}^{0}$ different from the $\not R p$ channel. In those kinds of scenarios where the LSP is not the $\tilde{\chi}_{1}^{0}$, the $\tilde{\chi}_{1}^{0} l_{i}^{ \pm}$production would not represent systematically the main contribution to the like sign dilepton signature.

In the several scenarios described above where the $\tilde{\chi}_{1}^{0} l_{i}^{ \pm}$production is not the major contribution to the like sign dilepton signature, this signature could receive quite important contribution from the other single gaugino productions described in Section 3.1. 


\section{Conclusion}

The single gaugino productions at Tevatron reach important cross sections thanks to the contributions of the resonant slepton productions. Hence, the analysis of the 3 charged leptons and like sign dilepton signatures generated by the single gaugino productions at Tevatron Run II would allow to obtain high sensitivities on many $R_{p}$ coupling constants, compared to the low-energy limits, in wide domains of the SUSY parameter space. This is also due to the fact that the Standard Model backgrounds associated to the 3 charged leptons and like sign dilepton final states at Tevatron can be greatly suppressed.

From the supersymmetry discovery point of view, superpartner masses well beyond the present experimental limits could be tested through the analysis of the the 3 charged leptons and like sign dilepton signatures generated by the single gaugino productions at Tevatron Run II. If some of the $R_{p}$ coupling constants values were close to their lowenergy bounds, the single gaugino productions study based on the 3 charged leptons and like sign dilepton signatures would even allow to extend the region in the $m_{0}-m_{1 / 2}$ plane probed by the superpartner pair production analyses in the 3 charged leptons and like sign dilepton channels at Tevatron Run II. The reason is that the single superpartner production has a larger phase space factor than the superpartner pair production.

Besides, the 3 charged leptons and like sign dilepton signatures generated by the single gaugino productions at Tevatron Run II would allow to reconstruct in a model independent way the $\tilde{\chi}_{1}^{0}, \tilde{\chi}_{1}^{ \pm}, \tilde{\nu}_{L}$ and $\tilde{l}_{L}^{ \pm}$masses with a smaller combinatorial background than in the superpartner pair production analysis.

We end this summary by a comparison between the results obtained from the studies of the 3 charged lepton and like sign dilepton signatures generated by the single gaugino productions at Tevatron Run II. In the mSUGRA model, the like sign dilepton signature analysis would give rise to higher sensitivities on the SUSY parameters than the study of the 3 charged lepton final state. This comes notably from the fact that in mSUGRA, the $\tilde{\chi}_{1}^{0}$ is lighter than the $\tilde{\chi}_{1}^{ \pm}$so that the cross section of the $\tilde{\chi}_{1}^{0} l^{ \pm}$production, which is the main contribution to the like sign dilepton signature, reaches larger values than the

cross section of the $\tilde{\chi}_{1}^{ \pm} l^{\mp}$ production, representing the main contribution to the 3 charged lepton final state.

Other interesting prospective studies concerning hadronic colliders are the analyses of the single gaugino productions occuring through resonant squark productions via $\lambda^{\prime \prime}$ coupling constants which we will perform in the next future.

\section{Acknowledgments}

We would like to thank Emmanuelle Perez, Robi Peschanski and Auguste Besson for fruitful discussions and reading the manuscript. 


\section{A Formulas for spin summed amplitudes}

In this Appendix, we give the amplitudes for all the single productions of supersymmetric particle at hadronic colliders, which can receive a contribution from a slepton resonant production. These single productions occur via the $R_{p}$ coupling $\lambda_{i j k}^{\prime}$ and correspond to the four reactions, $q \bar{q} \rightarrow \tilde{\chi}_{a}^{+} \bar{\nu}_{i}, q \bar{q} \rightarrow \tilde{\chi}_{a}^{0} \bar{\nu}_{i}, q \bar{q} \rightarrow \tilde{\chi}_{a}^{0} \bar{l}_{i}, q \bar{q} \rightarrow \tilde{\chi}_{a}^{-} \bar{l}_{i}$. Each of those four processes receives contributions from both the t and $u$ channel (see Fig.1) and have charge conjugated diagrams. Note also that the contributions coming from the exchange of a right squark in the $\mathrm{u}$ channel involve the higgsino components of the gauginos. These contributions, in the case of the single chargino production, do not interfere with the $\mathrm{s}$ channel slepton exchange since the initial or final states are different (see Fig.1). In the following, we give the formulas for the probability amplitudes, squared and summed over the polarizations. Our notations closely follow the notations of [48]. In particular, the matrix elements $N_{i j}^{\prime}$ are defined in the basis of the photino and the zino, as in [48].

$$
\begin{aligned}
\left|M_{s}\left(u^{j} \bar{d}^{k} \rightarrow \tilde{\chi}_{a}^{+} \bar{\nu}_{i}\right)\right|^{2} & =\frac{\lambda_{i j k}^{\prime}{ }^{2} g^{2}\left|U_{a 1}\right|^{2}}{12\left(s-m_{\tilde{l}_{L}^{i}}^{2}\right)^{2}}\left(m_{u^{j}}^{2}+m_{d^{k}}^{2}-s\right)\left(m_{\tilde{\chi}_{a}^{+}}^{2}-s\right) \\
\left|M_{t}\left(u^{j} \bar{d}^{k} \rightarrow \tilde{\chi}_{a}^{+} \bar{\nu}_{i}\right)\right|^{2} & =\frac{\lambda_{i j k}^{\prime} g^{2}}{12\left(t-m_{\tilde{d}_{L}^{j}}^{2}\right)^{2}}\left(m_{d^{k}}^{2}-t\right)\left[\left(\left|U_{a 1}\right|^{2}+\frac{m_{u^{j}}^{2}\left|V_{a 2}\right|^{2}}{2 m_{W}^{2} \sin ^{2} \beta}\right)\left(m_{u^{j}}^{2}+m_{\tilde{\chi}_{a}^{+}}^{2}-t\right)\right. \\
& \left.-\frac{4 m_{u^{j}}^{2} m_{\tilde{\chi}_{a}^{+}} R e\left(U_{a 1} V_{a 2}\right)}{\sqrt{2} m_{W} \sin \beta}\right] \\
\left|M_{u}\left(u^{k} \bar{d}^{j} \rightarrow \tilde{\chi}_{a}^{+} \nu_{i}\right)\right|^{2} & =\frac{\lambda_{i j k}^{\prime} g^{2} m_{d^{k}}^{2}\left|U_{a 2}\right|^{2}}{24 m_{W}^{2} \cos \beta^{2}\left(u-m_{\tilde{d}_{R}^{k}}^{2}\right)^{2}}\left(m_{\tilde{\chi}_{a}^{+}}^{2}+m_{u^{k}}^{2}-u\right)\left(m_{d^{j}}^{2}-u\right) \\
2 \operatorname{Re}\left[M_{s} M_{t}^{*}\left(\tilde{\chi}_{a}^{+} \bar{\nu}_{i}\right)\right] & =\frac{\lambda_{i j k}^{\prime} g^{2}}{6\left(s-m_{\tilde{l}_{L}^{i}}^{2}\right)\left(t-m_{\tilde{d}_{L}^{j}}^{2}\right)}\left[\frac { | U _ { a 1 } | ^ { 2 } } { 2 } \left[\left(m_{u^{j}}^{2}+m_{\tilde{\chi}_{a}^{+}}^{2}-t\right)\left(m_{d^{k}}^{2}-t\right)\right.\right. \\
& \left.+\left(m_{u^{j}}^{2}+m_{d^{k}}^{2}-s\right)\left(m_{\tilde{\chi}_{a}^{+}}^{2}-s\right)-\left(m_{u^{j}}^{2}-u\right)\left(m_{\tilde{\chi}_{a}^{+}}^{2}+m_{d^{k}}^{2}-u\right)\right] \\
& \left.-\left(m_{d^{k}}^{2}-t\right) \frac{R e\left(U_{a 1} V_{a 2}\right) m_{\tilde{\chi}_{a}^{+}}^{2} m_{u^{j}}^{2}}{\sqrt{2} m_{W} \sin \beta}\right],
\end{aligned}
$$

where, $s=\left(p\left(u^{j}\right)-p\left(\bar{d}_{k}\right)\right)^{2}, t=\left(p\left(u^{j}\right)-p\left(\tilde{\chi}_{a}^{+}\right)\right)^{2}$ and $u=\left(p\left(\bar{d}^{j}\right)-p\left(\nu_{i}\right)\right)^{2}$.

$$
\begin{aligned}
\left|M_{s}\left(d_{j} \bar{d}_{k} \rightarrow \tilde{\chi}_{a}^{0} \bar{\nu}_{i}\right)\right|^{2} & =\frac{\lambda_{i j k}^{\prime}{ }^{2} g^{2}\left|N_{a 2}^{\prime}\right|^{2}}{24 \cos ^{2} \theta_{W}\left(s-m_{\tilde{\nu}_{L}^{i}}^{2}\right)^{2}}\left(s-m_{d^{k}}^{2}-m_{d^{j}}^{2}\right)\left(s-m_{\tilde{\chi}_{a}^{0}}^{2}\right) \\
\left|M_{t}\left(d_{j} \bar{d}_{k} \rightarrow \tilde{\chi}_{a}^{0} \bar{\nu}_{i}\right)\right|^{2} & =\frac{\lambda_{i j k}^{\prime} g^{2}}{6\left(t-m_{\tilde{d}_{L}^{j}}^{2}\right)^{2}}\left(m_{d^{k}}^{2}-t\right)\left[( m _ { d ^ { j } } ^ { 2 } + m _ { \tilde { \chi } _ { a } ^ { 0 } } ^ { 2 } - t ) \left(\frac{g^{2} m_{d^{j}}^{2}\left|N_{a 3}^{\prime}\right|^{2}}{4 m_{W}^{2} \cos ^{2} \beta}+\frac{e^{2}}{9}\left|N_{a 1}^{\prime}\right|^{2}\right.\right. \\
& \left.+\frac{g^{2}\left|N_{a 2}^{\prime}\right|^{2}\left(\sin ^{2} \theta_{W} / 3-1 / 2\right)^{2}}{\cos ^{2} \theta_{W}}-\frac{2 e g R e\left(N_{a 1}^{\prime} N_{a 2}^{\prime}\right)\left(\sin ^{2} \theta_{W} / 3-1 / 2\right)}{3 \cos \theta_{W}}\right) \\
& \left.+\frac{2 m_{\tilde{\chi}_{a}^{0}} m_{d^{j}}^{2} g}{m_{W} \cos \beta}\left(-\frac{e R e\left(N_{a 1}^{\prime} N_{a 3}^{\prime}\right)}{3}+\frac{g R e\left(N_{a 2}^{\prime} N_{a 3}^{\prime}\right)}{\cos \theta_{W}}\left(\frac{\sin ^{2} \theta_{W}}{3}-\frac{1}{2}\right)\right)\right] \\
\left|M_{u}\left(d_{j} \bar{d}_{k} \rightarrow \tilde{\chi}_{a}^{0} \bar{\nu}_{i}\right)\right|^{2} & =\frac{\lambda_{i j k}^{\prime}}{6\left(u-m_{\tilde{d}_{R}^{k}}^{2}\right)^{2}}\left(m_{d^{j}}^{2}-u\right)\left[( m _ { \tilde { \chi } _ { a } ^ { 0 } } ^ { 2 } + m _ { d ^ { k } } ^ { 2 } - u ) \left(\frac{g^{2} m_{d^{k}}^{2}\left|N_{a 3}^{\prime}\right|^{2}}{4 m_{W}^{2} \cos ^{2} \beta}+\frac{e^{2}\left|N_{a 1}^{\prime}\right|^{2}}{9}\right.\right. \\
& \left.+\frac{g^{2} \sin ^{4} \theta_{W}\left|N_{a 2}^{\prime}\right|^{2}}{9 \cos { }^{2} \theta_{W}}-\frac{2 e g R e\left(N_{a 1}^{\prime} N_{a 2}^{\prime}\right) \sin ^{2} \theta_{W}}{9 \cos \theta_{W}}\right) \\
& \left.-\frac{2 m_{\tilde{\chi}_{a}^{0}} m_{d^{k}}^{2} g}{m_{W} \cos \beta}\left(-\frac{e R e\left(N_{a 1}^{\prime} N_{a 3}^{\prime}\right)}{3}+\frac{g \sin ^{2} \theta_{W} \operatorname{Re}\left(N_{a 2}^{\prime} N_{a 3}^{\prime}\right)}{3 \cos \theta_{W}}\right)\right] \\
2 \operatorname{Re}\left[M_{s} M_{t}^{*}\left(\tilde{\chi}_{a}^{0} \bar{\nu}_{i}\right)\right] & =-\frac{m_{\tilde{\chi}_{a}^{0}} m_{d^{j}}^{2} g R e\left(N_{a 2}^{\prime} N_{a 3}^{\prime}\right)}{m_{W} \cos \beta} \\
& -\frac{\lambda_{i j k}^{\prime} g}{12 \cos \theta_{W}\left(s-m_{\tilde{\nu}_{L}^{i}}^{2}\right)\left(t-m_{\tilde{d}_{L}^{j}}^{2}\right)}\left[\left(m_{d^{k}}^{2}-t\right) \frac{m^{2}}{}\right.
\end{aligned}
$$




$$
\begin{aligned}
& +\left(-\frac{e R e\left(N_{a 1}^{\prime} N_{a 2}^{*}\right)}{3}+\frac{g\left|N_{a 2}^{\prime}\right|^{2}}{\cos \theta_{W}}\left(\frac{\sin ^{2} \theta_{W}}{3}-\frac{1}{2}\right)\right)\left[\left(m_{d^{j}}^{2}+m_{\tilde{\chi}_{a}^{0}}^{2}-t\right)\left(m_{d^{k}}^{2}-t\right)\right. \\
& \left.\left.+\left(m_{d^{j}}^{2}+m_{d^{k}}^{2}-s\right)\left(m_{\tilde{\chi}_{a}^{0}}^{2}-s\right)-\left(m_{\tilde{\chi}_{a}^{0}}^{2}+m_{d^{k}}^{2}-u\right)\left(m_{d^{j}}^{2}-u\right)\right]\right] \\
2 \operatorname{Re}\left[M_{t} M_{u}^{*}\left(\tilde{\chi}_{a}^{0} \bar{\nu}_{i}\right)\right]= & \frac{\lambda_{i j k}^{\prime}{ }^{2}}{6\left(u-m_{\tilde{d}_{R}^{k}}^{2}\right)\left(t-m_{\tilde{d}_{L}^{j}}^{2}\right)}\left[\left(m_{d^{k}}^{2}-t\right) \frac{g m_{\tilde{\chi}_{a}^{0}} m_{d^{j}}^{2}}{m_{W} \cos \beta}\left(\frac{g \sin ^{2} \theta_{W} \operatorname{Re}\left(N_{a 2}^{\prime} N_{a 3}^{\prime}\right)}{3 \cos \theta_{W}}-\frac{e R e\left(N_{a 1}^{\prime} N_{a 3}^{\prime}\right)}{3}\right)\right. \\
+ & {\left[\left(m_{d^{j}}^{2}-u\right)\left(m_{\tilde{\chi}_{a}^{0}}^{2}+m_{d^{k}}^{2}-u\right)+\left(m_{d^{k}}^{2}-t\right)\left(m_{d^{j}}^{2}+m_{\tilde{\chi}_{a}^{0}}^{2}-t\right)-\left(m_{\tilde{\chi}_{a}^{0}}^{2}-s\right)\left(m_{d^{j}}^{2}+m_{d^{k}}^{2}-s\right)\right] } \\
& \left.\left(-\frac{e g R e\left(N_{a 1}^{\prime} N_{a 2}^{\prime}\right)}{3 \cos \theta_{W}}\left(\frac{2 \sin ^{2} \theta_{W}}{3}-\frac{1}{2}\right)+\frac{e^{2}\left|N_{a 1}^{\prime}\right|^{2}}{9}+\frac{g^{2} \sin ^{2} \theta_{W}\left|N_{a 2}^{\prime}\right|^{2}}{3 \cos ^{2} \theta_{W} \theta_{W}}-\frac{1}{2}\right)\right) \\
& -\frac{m_{\tilde{\chi}_{a}^{0}} m_{d^{k}}^{2} g}{m_{W} \cos \beta}\left(-\frac{e R e\left(N_{a 1}^{\prime} N_{a 3}^{\prime}\right)}{3}+\frac{g R e\left(N_{a 2}^{\prime} N_{a 3}^{\prime}\right)}{\cos \theta_{W}}\left(\frac{\sin ^{2} \theta_{W}}{3}-\frac{1}{2}\right)\right)\left(m_{d^{j}}^{2}-u\right) \\
& \left.+\frac{m_{d^{j}}^{2} m_{d^{k}}^{2} g^{2}\left|N_{a 3}^{\prime}\right|^{2}}{2 m_{W}^{2} \cos ^{2} \beta}\left(m_{\tilde{\chi}_{a}^{0}}^{2}-s\right)\right] \\
= & \frac{\lambda_{i j k}^{\prime} g}{12 \cos \theta_{W}\left(s-m_{\tilde{\nu}_{L}^{i}}^{2}\right)\left(u-m_{\tilde{d}_{R}^{k}}^{2}\right)}\left[-\frac{m_{\tilde{\chi}_{a}^{0}} m_{d^{k}}^{2} g R e\left(N_{a 2}^{\prime} N_{a 3}^{\prime}\right)}{m_{W} \cos \beta}\left(m_{d^{j}}^{2}-u\right)\right. \\
& +\left(-\frac{e R e\left(N_{a 1}^{*} N_{a 2}^{\prime}\right)}{3}+\frac{\left|N_{a 2}^{\prime}\right|^{2} g \sin ^{2} \theta_{W}}{3 \cos \theta_{W}}\right)\left[\left(m_{d^{j}}^{2}+m_{d^{k}}^{2}-s\right)\left(m_{\tilde{\chi}_{a}^{0}}^{2}-s\right)\right. \\
& \left.\left.+\left(m_{\tilde{\chi}_{a}^{0}}^{2}+m_{d^{k}}^{2}-u\right)\left(m_{d^{j}}^{2}-u\right)-\left(m_{d^{j}}^{2}+m_{\tilde{\chi}_{a}^{0}}^{2}-t\right)\left(m_{d^{k}}^{2}-t\right)\right]\right]
\end{aligned}
$$

where, $s=\left(p\left(d^{j}\right)-p\left(\bar{d}_{k}\right)\right)^{2}, t=\left(p\left(d^{j}\right)-p\left(\tilde{\chi}_{a}^{0}\right)\right)^{2}$ and $u=\left(p\left(d^{j}\right)-p\left(\bar{\nu}_{i}\right)\right)^{2}$.

$$
\begin{aligned}
& \left|M_{s}\left(u_{j} \bar{d}_{k} \rightarrow \tilde{\chi}_{a}^{0} \bar{l}_{i}\right)\right|^{2}=\frac{\lambda_{i j k}^{\prime}{ }^{2}}{6\left(s-m_{\tilde{l}_{L}^{i}}^{2}\right)^{2}}\left(s-m_{u^{j}}^{2}-m_{d^{k}}^{2}\right)\left[\left(\frac{g^{2} m_{l^{i}}^{2}\left|N_{a 3}^{\prime}\right|^{2}}{4 m_{W}^{2} \cos ^{2} \beta}+e^{2}\left|N_{a 1}^{\prime}\right|^{2}+\frac{g^{2}\left|N_{a 2}^{\prime}\right|^{2}}{\cos ^{2} \theta_{W}}\left(\sin ^{2} \theta_{W}-\frac{1}{2}\right)^{2}\right.\right. \\
& \left.-\frac{2 e g R e\left(N_{a 1}^{\prime} N_{a 2}^{\prime}\right)}{\cos \theta_{W}}\left(\sin ^{2} \theta_{W}-\frac{1}{2}\right)\right)\left(s-m_{l^{i}}^{2}-m_{\tilde{\chi}_{a}^{0}}^{2}\right)-\frac{2 g m_{\tilde{\chi}_{a}^{0}} m_{l^{i}}^{2}}{m_{W} \cos \beta}\left(-e \operatorname{Re}\left(N_{a 1}^{\prime} N_{a 3}^{\prime}\right)\right. \\
& \left.\left.+\frac{g R e\left(N_{a 2}^{\prime} N_{a 3}^{\prime}\right)}{\cos \theta_{W}}\left(\sin ^{2} \theta_{W}-\frac{1}{2}\right)\right)\right] \\
& \left|M_{t}\left(u_{j} \bar{d}_{k} \rightarrow \tilde{\chi}_{a}^{0} \bar{l}_{i}\right)\right|^{2}=\frac{\lambda_{i j k}^{\prime}{ }^{2}}{6\left(t-m_{\tilde{u}_{L}^{j}}^{2}\right)^{2}}\left(-t+m_{l^{i}}^{2}+m_{d^{k}}^{2}\right)\left[\left(\frac{g^{2} m_{u^{j}}^{2}\left|N_{a 4}^{\prime}\right|^{2}}{4 m_{W}^{2} \sin ^{2} \beta}+\frac{4 e^{2}\left|N_{a 1}^{\prime}\right|^{2}}{9}\right.\right. \\
& \left.+\frac{g^{2}\left|N_{a 2}^{\prime}\right|^{2}}{\cos ^{2} \theta_{W}}\left(\frac{1}{2}-\frac{2 \sin ^{2} \theta_{W}}{3}\right)^{2}+\frac{4 e g R e\left(N_{a 1}^{\prime} N_{a 2}^{\prime}\right)}{3 \cos \theta_{W}}\left(\frac{1}{2}-\frac{2 \sin ^{2} \theta_{W}}{3}\right)\right)\left(-t+m_{u^{j}}^{2}+m_{\tilde{\chi}_{a}^{0}}^{2}\right) \\
& \left.+\frac{2 g m_{u^{j}}^{2} m_{\tilde{\chi}_{a}^{0}}}{m_{W} \sin \beta}\left(\frac{2 e R e\left(N_{a 1}^{\prime} N_{a 4}^{\prime}\right)}{3}+\frac{g R e\left(N_{a 2}^{\prime} N_{a 4}^{\prime}\right)}{\cos \theta_{W}}\left(\frac{1}{2}-\frac{2 \sin ^{2} \theta_{W}}{3}\right)\right)\right] \\
& \left|M_{u}\left(u_{j} \bar{d}_{k} \rightarrow \tilde{\chi}_{a}^{0} \bar{l}_{i}\right)\right|^{2}=\frac{\lambda_{i j k}^{\prime}{ }^{2}}{6\left(u-m_{\tilde{d}_{R}^{k}}^{2}\right)^{2}}\left(m_{u^{j}}^{2}+m_{l^{i}}^{2}-u\right)\left[\left(\frac{e^{2}\left|N_{a 1}^{\prime}\right|^{2}}{9}+\frac{g^{2} \sin ^{4} \theta_{W}\left|N_{a 2}^{\prime}\right|^{2}}{9 \cos ^{2} \theta_{W}}-\frac{2 e g R e\left(N_{a 1}^{\prime} N_{a 2}^{\prime}\right) \sin ^{2} \theta_{W}}{9 \cos \theta_{W}}\right.\right. \\
& \left.+\frac{g^{2} m_{d^{k}}^{2}\left|N_{a 3}^{\prime}\right|^{2}}{4 m_{W}^{2} \cos ^{2} \beta}\right)\left(m_{\tilde{\chi}_{a}^{0}}^{2}+m_{d^{k}}^{2}-u\right)-\frac{2 g m_{d^{k}}^{2} m_{\tilde{\chi}_{a}^{0}}}{m_{W} \cos \beta}\left(-\frac{e R e\left(N_{a 1}^{\prime} N_{a 3}^{\prime}\right)}{3}\right. \\
& \left.\left.+\frac{g \sin ^{2} \theta_{W} \operatorname{Re}\left(N_{a 2}^{\prime} N_{a 3}^{\prime}\right)}{3 \cos \theta_{W}}\right)\right] \\
& 2 \operatorname{Re}\left[M_{s} M_{t}^{*}\left(\tilde{\chi}_{a}^{0} \bar{l}_{i}\right)\right]=-\frac{\lambda_{i j k}^{\prime}{ }^{2}}{6\left(s-m_{\tilde{l}_{L}^{i}}^{2}\right)\left(t-m_{\tilde{u}_{L}^{j}}^{2}\right)}\left[-\frac{m_{l^{i}}^{2} m_{u^{j}}^{2} g^{2} \operatorname{Re}\left(N_{a 3}^{\prime} N_{a 4}^{*}\right)}{2 m_{W}^{2} \sin \beta \cos \beta}\left(m_{\tilde{\chi}_{a}^{0}}^{2}+m_{d^{k}}^{2}-u\right)\right. \\
& +\left(\frac{-2 e^{2}\left|N_{a 1}^{\prime}\right|^{2}}{3}+\frac{e g R e\left(N_{a 1}^{*} N_{a 2}^{\prime}\right)}{3 \cos \theta_{W}}\left(4 \sin ^{2} \theta_{W}-\frac{5}{2}\right)\right. \\
& \left.+\frac{g^{2}\left|N_{a 2}^{\prime}\right|^{2}}{\cos ^{2} \theta_{W}}\left(\frac{1}{2}-\frac{2 \sin ^{2} \theta_{W}}{3}\right)\left(\sin ^{2} \theta_{W}-\frac{1}{2}\right)\right) \\
& {\left[\left(m_{u^{j}}^{2}+m_{d^{k}}^{2}-s\right)\left(m_{\tilde{\chi}_{a}^{0}}^{2}+m_{l^{i}}^{2}-s\right)+\left(m_{u^{j}}^{2}+m_{\tilde{\chi}_{a}^{0}}^{2}-t\right)\left(m_{l^{i}}^{2}+m_{d^{k}}^{2}-t\right)\right.} \\
& \left.-\left(m_{u^{j}}^{2}+m_{l^{i}}^{2}-u\right)\left(m_{\tilde{\chi}_{a}^{0}}^{2}+m_{d^{k}}^{2}-u\right)\right]+\frac{g m_{u^{j}}^{2} m_{\tilde{\chi}_{a}^{0}}}{m_{W} \sin \beta}\left(-e R e\left(N_{a 1}^{\prime} N_{a 4}^{\prime}\right)+\frac{g R e\left(N_{a 2}^{\prime} N_{a 4}^{\prime}\right)}{\cos \theta_{W}}\right.
\end{aligned}
$$




$$
\begin{aligned}
& \left.\left(\sin ^{2} \theta_{W}-\frac{1}{2}\right)\right)\left(m_{l^{i}}^{2}+m_{d^{k}}^{2}-t\right)-\left(s-m_{u^{j}}^{2}-m_{d^{k}}^{2}\right) \frac{g m_{l^{i}}^{2} m_{\tilde{\chi}_{a}^{0}}}{m_{W} \cos \beta}\left(\frac{2 e \operatorname{Re}\left(N_{a 1}^{\prime} N_{a 3}^{\prime}\right)}{3}\right. \\
& \left.\left.+\frac{g R e\left(N_{a 2}^{\prime} N_{a 3}^{\prime}\right)}{\cos \theta_{W}}\left(\frac{1}{2}-\frac{2 \sin ^{2} \theta_{W}}{3}\right)\right)\right] \\
& 2 \operatorname{Re}\left[M_{t} M_{u}^{*}\left(\tilde{\chi}_{a}^{0} \bar{l}_{i}\right)\right]=\frac{\lambda_{i j k}^{\prime}{ }^{2}}{6\left(u-m_{\tilde{d}_{R}^{k}}^{2}\right)\left(t-m_{\tilde{u}_{L}^{j}}^{2}\right)}\left[\frac { g m _ { u ^ { j } } ^ { 2 } m _ { \tilde { \chi } _ { a } ^ { 0 } } ^ { 0 } } { m _ { W } \operatorname { s i n } \beta } ( m _ { l ^ { i } } ^ { 2 } + m _ { d ^ { k } } ^ { 2 } - t ) \left(-\frac{e \operatorname{Re}\left(N_{a 1}^{\prime} N_{a 4}^{\prime}\right)}{3}\right.\right. \\
& \left.+\frac{g \sin ^{2} \theta_{W} R e\left(N_{a 2}^{\prime} N_{a 4}^{\prime}\right)}{3 \cos \theta_{W}}\right)-\frac{m_{\tilde{\chi}_{a}^{0}} g m_{d^{k}}^{2}}{m_{W} \cos \beta}\left(\frac{2 e \operatorname{Re}\left(N_{a 1}^{\prime} N_{a 3}^{\prime}\right)}{3}+\frac{g R e\left(N_{a 2}^{\prime} N_{a 3}^{\prime}\right)}{\cos \theta_{W}}\left(\frac{1}{2}-\frac{2 \sin ^{2} \theta_{W}}{3}\right)\right) \\
& \left(m_{l^{i}}^{2}+m_{u^{j}}^{2}-u\right)-\frac{g^{2} \operatorname{Re}\left(N_{a 3}^{\prime} N_{a 4}^{*}\right) m_{u^{j}}^{2} m_{d^{k}}^{2}}{2 m_{W}^{2} \cos \beta \sin \beta}\left(s-m_{l^{i}}^{2}-m_{\tilde{\chi}_{a}^{0}}^{2}\right)+\left(-\frac{2 e^{2}\left|N_{a 1}^{\prime}\right|^{2}}{9}\right. \\
& +\frac{e g R e\left(N_{a 1}^{*} N_{a 2}^{\prime}\right)}{3 \cos \theta_{W}}\left(-\frac{1}{2}+\frac{4 \sin ^{2} \theta_{W}}{3}\right)+\frac{g^{2} \sin ^{2} \theta_{W}\left|N_{a 2}^{\prime}\right|^{2}}{3 \cos ^{2} \theta_{W}} \\
& \left.\left(\frac{1}{2}-\frac{2 \sin ^{2} \theta_{W}}{3}\right)\right)\left[\left(m_{l^{i}}^{2}+m_{u^{j}}^{2}-u\right)\left(m_{\tilde{\chi}_{a}^{0}}^{2}+m_{d^{k}}^{2}-u\right)+\left(m_{l^{i}}^{2}+m_{d^{k}}^{2}-t\right)\left(m_{\tilde{\chi}_{a}^{0}}^{2}+m_{u^{j}}^{2}-t\right)\right. \\
& \left.\left.-\quad\left(m_{l^{i}}^{2}+m_{\tilde{\chi}_{a}^{0}}^{2}-s\right)\left(m_{d^{k}}^{2}+m_{u^{j}}^{2}-s\right)\right]\right] \\
& 2 R e\left[M_{s} M_{u}^{*}\left(\tilde{\chi}_{a}^{0} \bar{l}_{i}\right)\right]=\frac{\lambda_{i j k}^{\prime}{ }^{2}}{6\left(s-m_{\tilde{l}_{L}^{i}}^{2}\right)\left(u-m_{\tilde{d}_{R}^{k}}^{2}\right)}\left[-\frac{g m_{l^{i}}^{2} m_{\tilde{\chi}_{a}^{0}}}{m_{W} \cos \beta}\left(-\frac{e R e\left(N_{a 1}^{\prime} N_{a 3}^{\prime}\right)}{3}+\frac{g \sin ^{2} \theta_{W} \operatorname{Re}\left(N_{a 2}^{\prime} N_{a 3}^{\prime}\right)}{3 \cos \theta_{W}}\right)\right. \\
& \left(s-m_{d^{k}}^{2}-m_{u^{j}}^{2}\right)-\frac{g m_{d^{k}}^{2} m_{\tilde{\chi}_{a}^{0}}}{m_{W} \cos \beta}\left(-e \operatorname{Re}\left(N_{a 1}^{\prime} N_{a 3}^{\prime}\right)+\frac{g R e\left(N_{a 2}^{\prime} N_{a 3}^{\prime}\right)}{\cos \theta_{W}}\left(\sin \theta_{W}^{2}-\frac{1}{2}\right)\right) \\
& \left(m_{l^{i}}^{2}+m_{u^{j}}^{2}-u\right)+\frac{g^{2} m_{l^{i}}^{2} m_{d^{k}}^{2}\left|N_{a 3}^{\prime}\right|^{2}}{2 m_{W}^{2} \cos ^{2} \beta}\left(m_{\tilde{\chi}_{a}^{0}}^{2}+m_{u^{j}}^{2}-t\right)+\left(\frac{e^{2}\left|N_{a 1}^{\prime}\right|^{2}}{3}\right. \\
& \text { - } \left.\frac{e g R e\left(N_{a 1}^{*} N_{a 2}^{\prime}\right)}{3 \cos \theta_{W}}\left(2 \sin \theta_{W}^{2}-\frac{1}{2}\right)+\frac{g^{2}\left|N_{a 2}^{\prime}\right|^{2} \sin ^{2} \theta_{W}}{3 \cos ^{2} \theta_{W}}\left(\sin ^{2} \theta_{W}-\frac{1}{2}\right)\right) \\
& {\left[\left(m_{l^{i}}^{2}+m_{u^{j}}^{2}-u\right)\left(m_{\tilde{\chi}_{a}^{0}}^{2}+m_{d^{k}}^{2}-u\right)-\left(m_{l^{i}}^{2}+m_{d^{k}}^{2}-t\right)\left(m_{\tilde{\chi}_{a}^{0}}^{2}+m_{u^{j}}^{2}-t\right)\right.} \\
& \left.\left.+\left(m_{l^{i}}^{2}+m_{\tilde{\chi}_{a}^{0}}^{2}-s\right)\left(m_{d^{k}}^{2}+m_{u^{j}}^{2}-s\right)\right],\right]
\end{aligned}
$$

where, $s=\left(p\left(u^{j}\right)-p\left(\bar{d}_{k}\right)\right)^{2}, t=\left(p\left(u^{j}\right)-p\left(\tilde{\chi}_{a}^{0}\right)\right)^{2}$ and $u=\left(p\left(u^{j}\right)-p\left(\bar{l}_{i}\right)\right)^{2}$.

$$
\begin{aligned}
\left|M_{s}\left(d_{j} \bar{d}_{k} \rightarrow \tilde{\chi}_{a}^{-} \bar{l}_{i}\right)\right|^{2} & =\frac{g^{2} \lambda_{i j k}^{\prime}{ }^{2}}{6\left(s-m_{\tilde{\nu}_{L}^{i}}^{2}\right)^{2}}\left(s-m_{d^{j}}^{2}-m_{d^{k}}^{2}\right)\left[\left(\frac{m_{l^{i}}^{2}\left|U_{a 2}\right|^{2}}{4 m_{W}^{2} \cos ^{2} \beta}+\frac{\left|V_{a 1}\right|^{2}}{2}\right)\left(s-m_{\tilde{\chi}_{a}^{+}}^{2}-m_{l^{i}}^{2}\right)\right. \\
& \left.+\frac{\sqrt{2} \operatorname{Re}\left(V_{a 1} U_{a 2}\right) m_{l^{i}}^{2} m_{\tilde{\chi}_{a}^{+}}}{m_{W} \cos \beta}\right] \\
\left|M_{t}\left(d_{j} \bar{d}_{k} \rightarrow \tilde{\chi}_{a}^{-} \bar{l}_{i}\right)\right|^{2} & =\frac{g^{2} \lambda_{i j k}^{\prime}{ }^{2}}{3\left(t-m_{\tilde{u}_{L}^{j}}^{2}\right)^{2}}\left(t-m_{d^{k}}^{2}-m_{l^{i}}^{2}\right)\left[\left(t-m_{\tilde{\chi}_{a}^{+}}^{2}-m_{d^{j}}^{2}\right)\left(\frac{\left|V_{a 1}\right|^{2}}{4}+\frac{m_{d^{j}}^{2}\left|U_{a 2}\right|^{2}}{8 M_{W}^{2} \cos ^{2} \beta}\right)\right. \\
& \left.+\frac{\operatorname{Re}\left(V_{a 1} U_{a 2}\right) m_{\tilde{\chi}_{a}^{+}} m_{d^{j}}^{2}}{\sqrt{2} m_{W} \cos \beta}\right] \\
\left|M_{u}\left(\bar{u}_{k} u_{j} \rightarrow \tilde{\chi}_{a}^{-} \bar{l}_{i}\right)\right|^{2} & =\frac{g^{2} \lambda_{i j k}^{\prime}}{24\left(u-m_{\tilde{d}_{R}^{k}}^{2}\right)^{2}}\left(m_{\tilde{\chi}_{a}^{+}}^{2}+m_{u^{k}}^{2}-u\right)\left(m_{l^{i}}^{2}+m_{u^{j}}^{2}-u\right) \frac{\left|U_{a 2}\right|^{2} m_{d^{k}}^{2}}{m_{W}^{2} \cos ^{2} \beta} \\
2 R e\left[M_{s} M_{t}^{*}\left(\tilde{\chi}_{a}^{-} \bar{l}_{i}\right)\right] & =\frac{g^{2} \lambda_{i j k}^{\prime}{ }^{2}}{12\left(s-m_{\tilde{\nu}_{L}^{i}}^{2}\right)\left(t-m_{\tilde{u}_{L}^{j}}^{2}\right)}\left[| V _ { a 1 } | ^ { 2 } \left[-\left(m_{l^{i}}^{2}+m_{d^{j}}^{2}-u\right)\left(m_{\tilde{\chi}_{a}^{+}}^{2}+m_{d^{k}}^{2}-u\right)\right.\right. \\
& \left.+\left(m_{l^{i}}^{2}+m_{d^{k}}^{2}-t\right)\left(m_{\tilde{\chi}_{a}^{+}}^{2}+m_{d^{j}}^{2}-t\right)+\left(m_{l^{i}}^{2}+m_{\tilde{\chi}_{a}^{+}}^{2}-s\right)\left(m_{d^{k}}^{2}+m_{d^{j}}^{2}-s\right)\right] \\
& +\frac{R e\left(V_{a 1} U_{a 2}\right) m_{\tilde{\chi}_{a}^{+}} \sqrt{2}}{m_{W^{\prime}} \cos \beta}\left[m_{l^{i}}^{2}\left(s-m_{d^{j}}^{2}-m_{d^{k}}^{2}\right)-m_{d^{j}}^{2}\left(m_{l^{i}}^{2}+m_{d^{k}}^{2}-t\right)\right] \\
& \left.-\frac{\left|U_{a 2}\right|^{2} m_{l^{i}}^{2} m_{d^{j}}^{2}}{m_{W}^{2} \cos ^{2} \beta}\left(m_{\tilde{\chi}_{a}^{+}}^{2}+m_{d^{k}}^{2}-u\right)\right],
\end{aligned}
$$

where, $s=\left(p\left(d^{j}\right)-p\left(\bar{d}_{k}\right)\right)^{2}, t=\left(p\left(d^{j}\right)-p\left(\tilde{\chi}_{a}^{-}\right)\right)^{2}$ and $u=\left(p\left(u^{j}\right)-p\left(\bar{l}_{i}\right)\right)^{2}$. 


\section{References}

[1] S. Dimopoulos and L.J. Hall, Phys. Lett. B 207, 210 (1988).

[2] H. Dreiner and G. G. Ross, Nucl. Phys. B 365, 597 (1991).

[3] H. Dreiner, published in Perspectives on Supersymmetry, ed. by G.L. Kane, World Scientific (1998), hep-ph/9707435.

[4] G. Bhattacharyya, Invited talk presented at 'Beyond the Desert', Castle Ringberg, Tegernsee, Germany, 8-14 June 1997; Susy '96, Nucl. Phys. B (Proc. Suppl.) 52A 83 (1997).

[5] R. Barbier et al., Report of the Group on the R-parity Violation, hep-ph/9810232.

[6] V. Barger, G. F. Giudice and T. Han, Phys. Rev. D 40, 2987 (1989).

[7] S. Dimopoulos, R. Esmailzadeh, L.J. Hall, J. Merlo and G.D. Starkman, Phys. Rev. D41, 2099 (1990).

[8] P. Binétruy et al., ECFA Large Hadron Collider (LHC) Workshop, Aachen, 1990, Vol. II.

[9] A. Datta, J. M. Yang, B.-L. Young and X. Zhang, Phys. Rev. D56, 3107 (1997).

[10] R. J. Oakes, K. Whisnant, J. M. Yang, B.-L. Young and X. Zhang, Phys. Rev. D57, 534 (1998).

[11] J. L. Hewett and T. G. Rizzo, "Proceedings of the XXIX International Conference on High Energy Physics", Vancouver, CA, 23-29 July 1998, hep-ph/9809525.

[12] P. Chiappetta, A. Deandrea, E. Nagy, S. Negroni, G. Polesello and J.M. Virey, Phys. Rev. D61, 115008 (2000).

[13] J. Kalinowski, R. Rückl, H. Spiesberger and P. M. Zerwas, Phys. Lett. B414, 297 (1997).

[14] J. Kalinowski, Proceedings of "Beyond the Desert 97 - Accelerator and NonAccelerator Approaches", Ringberg Castle, Germany, June 1997, hep-ph/9708490.

[15] S. Bar-Shalom, G. Eilam and A. Soni, Phys. Rev. D59, 055012 (1999).

[16] S. Lola, Presented at the 2nd ECFA/DESY study on Linear Colliders, Frascati, November 1998, LC note LC-TH-1999-021, hep-ph/9912217.

[17] L. E. Ibañez and G. G. Ross, CERN-TH.6412/92, in Perspectives in Higgs Physics, G. Kane editor, World Scientific, Singapore, 1993.

[18] H. Dreiner, P. Richardson and M. H. Seymour, to appear in the proceedings of the BTMSSM subgroup of the Physics at Run II Workshop on Supersymmetry/Higgs, hep-ph/9903419.

[19] F. Déliot, G. Moreau, C. Royon, E. Perez and M. Chemtob, Phys. Lett. B475, 184 (2000).

[20] H. Dreiner, P. Richardson and M. H. Seymour, hep-ph/0001224. 
[21] G. Moreau, E. Perez and G. Polesello, hep-ph/0003012, subm. to Nucl. Phys. B; G. Moreau, E. Perez and G. Polesello, Proceedings of the Workshop 'Physics at TeV Colliders', 8-18 June, 1999, Les Houches, France, hep-ph/0002130.

[22] E. L. Berger, B. W. Harris and Z. Sullivan, Phys. Rev. Lett. 83, 4472 (1999).

[23] M. Carena, M. Olechowski, S. Pokorski and C. E. M. Wagner, Nucl. Phys. B419 (1994) 213.

[24] Top averaging group, preprint Fermilab TM-2084 (1999) D0 Collab., Phys. Rev. D58, 052001 (1998), Phys. Rev. Lett. 79, 1197 (1997), Phys. Rev. D60, 052001 (1999), CDF Collab., Phys. Rev. Lett. 80, 2767 (1998), Phys. Rev. Lett. 82, 271, (1999).

[25] CTEQ Coll., Phys. Rev. D55, 1280 (1997).

[26] R. Baier, J. Engels, B. Petersson, Z Phys. C2, 265 (1979).

[27] E. W. N. Glover, A. D. Martin, R. G. Roberts, W. J. Stirling, Phys. Lett. B381, 353 (1996).

[28] M. Glück, E. Reya, A. Vogt, Z Phys. C53, 127 (1992).

[29] ALEPH Collaboration, ALEPH 2000-013, CONF 010 (2000).

[30] C. Caso et al., The European Physical Journal C3, 1 (1998).

[31] A. Pukhov, E. Boos, M. Dubinin, V. Edneral, V. Ilyin, D. Kovalenko, A. Kryukov, V. Savrin, S. Shichanin, and A. Semenov, "CompHEP - a package for evaluation of Feynman diagrams and integration over multi-particle phase space. User's manual for version 33.", Preprint INP MSU 98-41/542, hep-ph/9908288.

[32] T. Sjöstrand, Comp. Phys. Comm. 82, 74 (1994); S. Mrenna, Comp. Phys. Comm. 101, 232 (1997).

[33] J. Lykken and K. T. Matchev, preprint FERMILAB-PUB-99/034-T, Phys. Rev. D61, 015001 (2000).

[34] K. T. Matchev and D. M. Pierce, preprint FERMILAB-PUB-99/078-T, Phys. Rev. D60, 075004 (1999).

[35] H. Baer, M. Drees, F. Paige, P. Quintana, X. Tata, hep-ph/9906233.

[36] R. Barbieri, F. Caravaglios, M. Frigeni and M. Mangano, Nucl. Phys. B 367, 28 (1991).

[37] J. Conway, talk given at the SUSY/Higgs Workshop Meeting, Fermilab, May 14-16, 1998.

See also www.physics.rutgers.edu/jconway/soft/shw/shw.html.

[38] T. Kamon, J. L. Lopez, P. McIntyre, and J. T. White, Phys. Rev. D50, 5676 (1994).

[39] ATLAS Coll., 'ATLAS Detector and Physics Performance Technical Design Report', Vol. II, ATLAS TDR 15, 25 May 1999, CERN/LHCC 99-15.

See also atlasinfo.cern.ch/Atlas/GROUPS/PHYSICS/TDR/access.html. 
[40] B. Allanach et al., 'Searching for R-Parity Violation at Run-II of Tevatron', hepph/9906224.

[41] V. Barger et al., Phys. Rev. D50, 4299 (1994).

[42] H. Baer, C. Kao and X. Tata, Phys. Rev. D51, 2180 (1995).

[43] H. Baer, C.-H. Chen and X. Tata, Phys. Rev. D55, 1466 (1997).

[44] HERWIG 6.1 Release Note, hep-ph/9912396; G. Marchesini, B. R. Webber, G. Abbiendi, I. G. Knowles, M. H. Seymour and L. Stanco, Computer Phys. Commun. 67, 465 (1992).

[45] SUSYGEN 3.0/06, 'A Monte Carlo Event generator for MSSM sparticle production for $e^{+} e^{-}, \mu^{+} \mu^{-}$and ep colliders', N. Ghodbane, S. Katsanevas, P. Morawitz and E. Perez, lyoinfo.in2p3.fr/susygen/susygen3.html; N. Ghodbane, hep-ph/9909499.

[46] E. Perez, private communication.

[47] ALEPH Collaboration, ALEPH 98-017, CONF 007 (1998).

[48] J. F. Gunion and H. E. Haber, Nucl. Phys. B272, 1 (1986); Erratum-ibid B402, 567 (1993). 